\title{
The Grassmannian-like coset model and the higher spin currents
}

\author{
Changhyun Ahn \\ Department of Physics, Kyungpook National University, \\ Taegu 41566, Korea \\ E-mail: ahn@mail.knu.ac.kr
}

ABStraCt: In the Grassmannian-like coset model, $\frac{\mathrm{SU}(N+M)_{k}}{\mathrm{SU}(N)_{k} \times \mathrm{U}(1)_{k N M(N+M)}}$, Creutzig and Hikida have found the charged spin-2, 3 currents and the neutral spin-2, 3 currents previously. In this paper, as an extension of Gaberdiel-Gopakumar conjecture found ten years ago, we calculate the operator product expansion (OPE) between the charged spin-2 current and itself, the OPE between the charged spin- 2 current and the charged spin- 3 current and the OPE between the neutral spin-3 current and itself for generic $N, M$ and $k$. From the second OPE, we obtain the new charged quasi primary spin- 4 current while from the last one, the new neutral primary spin- 4 current is found implicitly. The infinity limit of $k$ in the structure constants of the OPEs is described in the context of asymptotic symmetry of $M \times M$ matrix generalization of $\mathrm{AdS}_{3}$ higher spin theory. Moreover, the OPE between the charged spin-3 current and itself is determined for fixed $(N, M)=(5,4)$ with arbitrary $k$ up to the third order pole. We also obtain the OPEs between charged spin-1,2,3 currents and neutral spin-3 current. From the last OPE, we realize that there exists the presence of the above charged quasi primary spin- 4 current in the second order pole for fixed $(N, M)=(5,4)$. We comment on the complex free fermion realization.

Keywords: AdS-CFT Correspondence, Conformal and W Symmetry, Higher Spin Gravity, Higher Spin Symmetry

ARXIV EPRINT: 2011.11240

On the occasion of my sixtieth birthday. 


\section{Contents}

1 Introduction 1

2 Review with some new derivations 4

2.1 A charged spin 2 current 6

2.2 A charged spin 3 current 8

$\begin{array}{ll}2.3 \text { An uncharged spin } 3 \text { current } & 12\end{array}$

3 The OPE between the charged higher spin-2 current and itself $\quad 15$

$\begin{array}{ll}3.1 \text { The fourth, third and second order poles } & 16\end{array}$

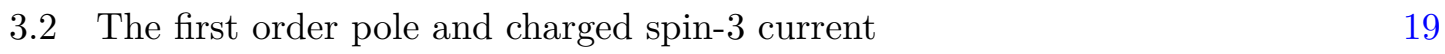

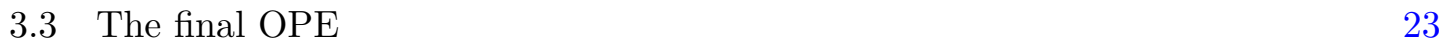

4 The OPE between the charged higher spin-2 current and the charged higher spin-3 current

4.1 The fifth, fourth and third order poles 25

$\begin{array}{ll}4.2 \text { The second order pole } & 28\end{array}$

4.2.1 Complete second order pole in the coset realization 28

4.2.2 How to rearrange the second order pole 28

4.3 The first order pole and charged quasi primary spin-4 current 33

$\begin{array}{lll}4.4 & \text { The final OPE } & 36\end{array}$

5 The OPE between the charged spin-3 current and itself with

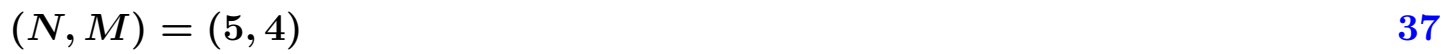

5.1 The sixth, fifth, fourth order poles $\quad 37$

$\begin{array}{ll}5.2 \text { The third order pole } & 37\end{array}$

$\begin{array}{ll}5.3 & \text { The second and first order poles } \\ \end{array}$

6 The OPE between the uncharged higher spin-3 current and itself $\quad 38$

6.1 For fixed $(N, M)=(5,4)$ case 38

6.1.1 The sixth, fifth, fourth and third order poles 38

6.1.2 The second and first order poles with the presence of uncharged primary spin-4 current $\quad 39$

$\begin{array}{lll}6.2 & \text { For general }(N, M) \text { case } & 40\end{array}$

7 The OPE between the charged (higher) spin currents and the uncharged higher spin-3 current

7.1 The OPE $J^{a}(z) W^{(3)}(w) \quad 41$

$\begin{array}{lll}7.2 & \text { The OPE } K^{a}(z) W^{(3)}(w) & 42\end{array}$

7.3 The OPE $P^{a}(z) W^{(3)}(w)$ with $(N, M)=(5,4)$

7.3.1 The sixth, fifth, fourth and third order poles 43

$\begin{array}{lll}\text { 7.3.2 The second and first order poles } & 43\end{array}$ 
A An SU(M) invariant tensors in terms of Kronecker delta, $f$ and $d$ symbols

B The first order pole in the OPE between the charged spin-2 current and itself

B.1 The substitution of charged spin-2 current 48

B.2 The adjoint spin-1 dependent terms in the first order pole 50

B.3 The first order pole $\quad 51$

C The structure constants in the infinity limit of $k$ of section $3 \quad 52$

D The second order pole in the $\operatorname{OPE} K^{a}(z) P^{b}(w) \quad 52$

D.1 The $a_{5}$ terms of the second order pole $\quad 53$

D.2 The $\left(a_{13}-a_{12}\right)$ terms of the second order pole 55

D.3 The relations between the remaining coefficients of $W^{(3)}(w)$ in the second $\begin{array}{ll}\text { order pole } & 58\end{array}$

D.4 The relations between the remaining coefficients of $P^{b}(w)$ in the second order pole

E The first order pole in the $\operatorname{OPE} K^{a}(z) P^{b}(w) \quad 60$

F The second order pole in the $\operatorname{OPE} K^{a}(z) W^{(3)}(w) \quad 61$

G The first order pole in the $\operatorname{OPE} K^{a}(z) W^{(3)}(w) \quad 62$

H Relevant free field realization $\quad 63$

H.1 Free field construction $\quad 64$

H.2 After decoupling the neutral spin-1 current 66

\section{Introduction}

The Grassmannian-like coset model is described by [1]

$$
\frac{\mathrm{SU}(N+M)_{k}}{\mathrm{SU}(N)_{k} \times \mathrm{U}(1)_{k N M(N+M)}} .
$$

By introducing the 't Hooft-like coupling constant $\lambda \equiv \frac{k}{(k+N)}$ and taking the infinity limit of $N$ with fixed $\lambda$ and $M$, it has been proposed in [2] that the above coset model is dual to $M \times M$ matrix generalization of $\mathrm{AdS}_{3}$ Vasiliev higher spin theory [3, 4]. For $M=1$, their proposal leads to the Gaberdiel-Gopakumar conjecture [5] via level-rank duality. See also [6-8] for review of [5]. The central charge of the coset model with infinity limit of level $k$ with fixed $\lambda$ and $M$ coincides with the one in the asymptotic symmetry of above 
$\mathrm{AdS}_{3}$ higher spin theory. The charged spin-2, 3 currents and the neutral (higher) spin-2,3 current in terms of the coset realization characterized by five spin- 1 currents have been found explicitly. At $\lambda=2$ ( or $k=-2 N$ ), the operator product expansion (OPE) between the charged spin-2 current and itself for general $(N, M)$, by decoupling the charged spin-3 current, leads to the one of the "rectangular" $W$-algebra with $\mathrm{SU}(M)$ symmetry of $\mathrm{AdS}_{3}$ higher spin theory.

In this paper, we will compute the OPE between the charged spin-2 current and itself by hand, for generic $k$ as well as generic $N$ and $M$. It turns out that the above charged spin3 current, for generic $\lambda$, should appear in the right hand side of the OPE. The structure constants appearing in the right hand side of this OPE in terms of these three parameters will be determined completely.

At each singular term, we should rearrange the coset composite operators in terms of the known currents, i) the stress energy tensor of spin-2, ii) the spin-1 current of $\mathrm{SU}(M)$, iii) the charged spin-2 current by allowing all the possible nonlinear terms.

It is known that after subtracting the descendant terms, we are left with the sum of quasi primary operators [9-11]. We should determine the structure constants appearing in these quasi primary operators of the right hand side of the OPE. Because there are free adjoint indices $a$ and $b$ of $\mathrm{SU}(M)$ in the left hand side of the OPE, it is rather nontrivial to exhaust all the possible quasi primary operators which will be contracted with some $\mathrm{SU}(M)$ invariant tensors. For example, in general, the first order pole of this OPE can contain the cubic terms in the spin- 1 current which possesses a single adjoint index. Then those invariant tensors will contain fifth order invariant ones maximally. That is, two of them will be the above free indices while three of them will be contracted with each index of cubic terms. This is the reason why the OPE between the nonsinglet charged operators even their spins are low is more complicated to analyze, compared to the OPE between the singlet operators. Note that in the examples of [12-14], there exist some OPEs having nonsinglet indices associated with the $\mathrm{SO}(4)$ but for these cases it is not so difficult to figure out its structures in the right hand sides of the OPEs because we can determine the vector and adjoint indices and the invariant tensors in $\mathrm{SO}(4)$ for fixed rank.

Furthermore, we will obtain the OPE between the charged spin- 2 current and the charged spin-3 current which occurs at the first order pole of the previous OPE between the charged spin- 2 current and itself. Now we should include both the charged spin-3 current and the neutral spin-3 current as the candidates for the quasi primary operators in the list of known currents we described in previous paragraph. The presence of the neutral spin-3 current is due to the fact that the left hand side of this OPE has two different operators, contrary to the previous OPE between the charged spin- 2 current and itself. The point is how we can write down the singular terms described by the coset realization in terms of the known currents. We expect that up to the second order pole of this OPE, we should express them by using the known currents with various $\mathrm{SU}(M)$ invariant tensors.

By analyzing the first order pole of this OPE, we will determine the new quasi primary charged spin-4 current in terms of coset realization. By construction, all the relative coefficients appearing in the coset composite operators are determined automatically although the careful analysis should be performed. 
From the explicit result for the OPE between the neutral spin-3 current and itself for fixed $(N, M)$ values, we will extract this OPE for generic $(N, M)$ case and at the second order pole of this OPE we will observe that there should be new primary neutral spin- 4 current in terms of coset realization.

In obtaining this result, we realize that the $k$-dependent structure constant can be rewritten as the modified central charge which is equal to the coset central charge subtracted by the central term due to the stress energy tensor for the quadratic Sugawara term in the spin-1 current of $\mathrm{SU}(M)$.

Then i) the modified stress energy tensor of spin-2, ii) the neutral spin-3 current and iii) the neutral spin- 4 current will consist of the generators of the standard $W$ algebra and their OPEs with the spin- 1 current do not have any singular terms. That is, the spin-1 current is decoupled in the OPEs between these singlet currents.

In section 2, we review the results of [2] by emphasizing that the spin-2 current and the spin-3 currents can be obtained by hands without trying to perform for several $(N, M)$ values. Those currents were determined previously. The derivations for obtaining these are new. In section 3, the simplest nontrivial OPE between the charged spin- 2 current and itself can be obtained. The structure constants are new. We will observe the charged spin-3 current at the first order pole. In section 4 , the next nontrivial OPE between the charged spin-2 current and the charged spin-3 current can be obtained. The new charged quasi primary spin- 4 current at the first order pole is determined.

In section 5, the new OPE between the charged spin-3 current and itself can be determined for specific $N$ and $M$ values. ${ }^{1}$ In section 6 , the new OPE between the uncharged spin- 3 current and itself can be determined and the new uncharged spin- 4 current appears at the second order pole. In section 7, the new OPEs between the charged spin-1,2, 3 currents and the uncharged spin-3 current are described. In section 8 , we present the future directions with a summary of this paper. In appendices, we will describe some detailed calculations based on the previous sections. The free field realization of [15] is reviewed and we explain how their results can be related to the previous results by taking the appropriate limits for the parameters we are considering.

The Thielemans package [16] is used together with the mathematica package [17]. The similar coset in the work of [18] where the possibility of four parameters in the specific coset is described is studied. ${ }^{2}$

\footnotetext{
${ }^{1}$ The integer $M=4$ is the lowest value in order to have an independent $\mathrm{SU}(M)$ invariant tensors [2]. We take $N$ which is different from $M$ as five.

${ }^{2}$ There is a similar construction, a matrix extended $W_{1+\infty}$ algebra [19], defined in terms of matrix extended Miura transformation (See also [20] for some mathematics for the "rectangular" $W$-algebra). The truncation of this matrix extended $W_{1+\infty}$ algebra can be realized the one in (1.1) without U(1) factor in the denominator. The three parameters of the algebra are given by $N, M$ and $k$ in the subsection 3.5 of [19]. We thank Lorenz Eberhardt for pointing this out.
} 
The charged spin-1,2,3,4 currents and uncharged spin-2,3,4 currents we are considering in this paper are given by

$$
\begin{array}{llll}
\operatorname{spin}-1: J^{a}(z), & \operatorname{spin}-2: K^{a}(z), & \operatorname{spin}-3: P^{a}(z), & \operatorname{spin}-4: \hat{R}^{a}(z), \\
\operatorname{spin}-2: T(z), & \operatorname{spin}-3: W^{(3)}(z), & & \operatorname{spin}-4: W^{(4)}(z) .
\end{array}
$$

Here $T(z)$ is the stress energy tensor and the index $a$ in (1.2) is an adjoint index of $\operatorname{SU}(M)$ and $a=1,2, \cdots,\left(M^{2}-1\right)$. Except of $T(z)$ and $\hat{R}^{a}(z)$ which are quasi primary currents, the remaining currents are primary ones under the stress energy tensor. In the context of [5], the OPEs between the neutral higher spin currents are relevant to this conjecture and the algebra between them is closed under the neutral higher spin currents. In addition to that, there are also the OPEs between the charged higher spin currents and the neutral ones and the OPEs between the charged higher spin currents. The right hand sides of these OPEs will contain the composite charged or neutral higher spin operators.

The main work of this paper is to start with the charged and neutral higher spin currents [2] and construct their algebra explicitly as an extension of [5] in the above coset model (1.1).

What we have found newly in this paper is the higher spin-4 currents in (1.2). The remaining ones were found in [2] previously.

\section{Review with some new derivations}

The normalization of the generators $\left(t^{\alpha}, t^{a}, t^{u(1)}, t^{(\rho \bar{i})}, t^{(\bar{\sigma} j)}\right)$ in $\mathrm{SU}(N+M)$ of the coset (1.1) can be fixed by taking the following simple metric [2]

$$
\operatorname{Tr}\left(t^{\alpha} t^{\beta}\right)=\delta^{\alpha \beta}, \quad \operatorname{Tr}\left(t^{a} t^{b}\right)=\delta^{a b}, \quad \operatorname{Tr}\left(t^{u(1)} t^{u(1)}\right)=1, \quad \operatorname{Tr}\left(t^{(\rho \bar{i})} t^{(\bar{\sigma} j)}\right)=\delta^{\rho \bar{\sigma}} \delta^{j \bar{i}} .
$$

Under the decomposition of $\mathrm{SU}(N+M)$ into the $\mathrm{SU}(N) \times \mathrm{SU}(M)$, the adjoint representation of $\mathrm{SU}(N+M)$ breaks into

$$
(\mathbf{N}+\mathbf{M})^{2}-\mathbf{1} \longrightarrow\left(\mathbf{N}^{2}-\mathbf{1}, \mathbf{1}\right) \oplus\left(\mathbf{1}, \mathbf{M}^{2}-\mathbf{1}\right) \oplus(\mathbf{1}, \mathbf{1}) \oplus(\mathbf{N}, \overline{\mathbf{M}}) \oplus(\overline{\mathbf{N}}, \mathbf{M}) .
$$

The fundamental indices $\rho$ and $j$ among (2.2) run over $\rho=1,2, \cdots, N$ and $j=1,2, \cdots, M$, while the antifundamental indices $\bar{\sigma}$ and $\bar{i}$ run over $\bar{\sigma}=1,2, \cdots, N$ and $\bar{i}=1,2, \cdots, M$. Note that the barred index in (2.1) becomes the unbarred one when we raise or lower it and vice versa. For the $\alpha, a$ and $u(1)$ indices where the adjoint indices are given by $\alpha=1,2, \cdots,\left(N^{2}-1\right)$ and $a=1,2, \cdots,\left(M^{2}-1\right)$ respectively, we can raise or lower them without any change. ${ }^{3}$ We will use the metric in (2.1) all the time.

For the above given generators, the totally antisymmetric $f$ and totally symmetric $d$ symbols can be expressed as follows:

$$
\begin{array}{ll}
\operatorname{Tr}\left(\left[t^{\alpha}, t^{\beta}\right] t^{\gamma}\right)=i f^{\alpha \beta \gamma}, & \operatorname{Tr}\left(\left[t^{a}, t^{b}\right] t^{c}\right)=i f^{a b c}, \quad \operatorname{Tr}\left(\left\{t^{\alpha}, t^{\beta}\right\} t^{\gamma}\right)=d^{\alpha \beta \gamma}, \\
\operatorname{Tr}\left(\left\{t^{a}, t^{b}\right\} t^{c}\right)=d^{a b c}, & \cdots,
\end{array}
$$

\footnotetext{
${ }^{3}$ Sometimes we use the $\mathrm{SU}(M)$ indices $a, b, c, \cdots$ as superscripts.
} 
where the abbreviated parts can be written similarly. We use the following nontrivial $f$ symbols [2] which are totally antisymmetric

$$
\begin{aligned}
i f^{(\overline{\rho i})(\bar{\sigma} j) u(1)} & =\sqrt{\frac{M+N}{M N}} \delta^{j \bar{i}} \delta^{\rho \bar{\sigma}}, \quad i f^{(\overline{\rho i})(\bar{\sigma} j) \alpha}=\delta^{\rho \overline{\rho_{1}}} \delta^{\sigma_{1} \bar{\sigma}} \delta^{j \bar{i}} t_{\sigma_{1} \overline{\rho_{1}}}^{\alpha}, \\
i f^{(\bar{\rho} \bar{i})(\bar{\sigma} j) a} & =-\delta^{\rho \bar{\sigma}} \delta^{i_{1} \bar{i}} \delta^{j \overline{j_{1}}} t_{i_{1} \bar{j}_{1}}^{a} .
\end{aligned}
$$

Due to the traceless property of the generators, when the indices $\rho$ and $\bar{\sigma}$ are equal to each other in the second relation of (2.4), the corresponding $f$ symbols are zero. Similarly, for the equal $\bar{i}$ and $j$ in the third relation, the $f$ symbols vanish.

The nontrivial $\mathrm{SU}(N+M)$ currents satisfy the following OPEs [2]

$$
\begin{aligned}
& J^{\alpha}(z) J^{\beta}(w)=\frac{1}{(z-w)^{2}} k \delta^{\alpha \beta}+\frac{1}{(z-w)} i f_{\gamma}^{\alpha \beta} J^{\gamma}(w)+\cdots, \\
& J^{\alpha}(z) J^{(\rho \bar{i})}(w)=\frac{1}{(z-w)} i f_{(\sigma \bar{j})}^{\alpha(\overline{\bar{i}})} J^{(\sigma \bar{j})}(w)+\cdots, \\
& J^{\alpha}(z) J^{(\bar{\rho} i)}(w)=\frac{1}{(z-w)} i f_{(\bar{\sigma} j)}^{\alpha(\bar{\rho})} J^{(\bar{\sigma} j)}(w)+\cdots, \\
& J^{a}(z) J^{b}(w)=\frac{1}{(z-w)^{2}} k \delta^{a b}+\frac{1}{(z-w)} i f_{c}^{a b} J^{c}(w)+\cdots, \\
& J^{a}(z) J^{(\rho \bar{i})}(w)=\frac{1}{(z-w)} i f_{(\sigma \bar{j})}^{a(\overline{\bar{i}})} J^{(\sigma \bar{j})}(w)+\cdots, \\
& J^{a}(z) J^{(\bar{\rho} i)}(w)=\frac{1}{(z-w)} i f_{(\bar{\sigma} j)}^{a(\bar{\rho})} J^{(\bar{\sigma} j)}(w)+\cdots, \\
& J^{u(1)}(z) J^{u(1)}(w)=\frac{1}{(z-w)^{2}} k+\cdots, \\
& J^{u(1)}(z) J^{(\rho \bar{i})}(w)=\frac{1}{(z-w)} i f_{(\sigma \bar{j})}^{u(1)(\rho \bar{i})} J^{(\sigma \bar{j})}(w)+\cdots, \\
& J^{u(1)}(z) J^{(\bar{\rho} i)}(w)=\frac{1}{(z-w)} i f_{(\bar{\sigma} j)}^{u(1)(\bar{\rho} i)} J^{(\bar{\sigma} j)}(w)+\cdots, \\
& J^{(\rho \bar{i})}(z) J^{(\bar{\sigma} j)}(w)=\frac{1}{(z-w)^{2}} k \delta^{\rho \bar{\sigma}} \delta^{j \bar{i}} \\
& +\frac{1}{(z-w)}\left[i f_{u(1)}^{(\rho \bar{i})(\bar{\sigma} j)} J^{u(1)}+i f_{\alpha}^{(\rho \bar{i})(\bar{\sigma} j)} J^{\alpha}+i f_{a}^{(\bar{\rho} \bar{i})(\bar{\sigma} j)} J^{a}\right](w)+\cdots .
\end{aligned}
$$

The second order pole in (2.5) has the explicit $k$ dependence with weight 1 . From the nonzero $f$ symbols in (2.4), the spin-1 currents transforming as $(\mathbf{N}, \overline{\mathbf{M}})$ or $(\overline{\mathbf{N}}, \mathbf{M})$ appear in many places of (2.5). Due to the last OPE in (2.5), the contraction between the spin-1 current and its conjugated one in the OPEs later will provide the remaining three kinds of spin-1 currents in the right hand side.

Note that there are also the five regular OPEs besides the above ten OPEs

$$
\begin{aligned}
J^{\alpha}(z) J^{a}(w) & =0+\cdots, \quad J^{\alpha}(z) J^{u(1)}(w)=0+\cdots, \quad J^{a}(z) J^{u(1)}(w)=0+\cdots, \\
J^{(\rho \bar{i})}(z) J^{(\sigma \bar{j})}(w) & =0+\cdots, \quad J^{(\bar{\rho} i)}(z) J^{(\bar{\sigma} j)}(w)=0+\cdots .
\end{aligned}
$$


These come from the trivial results from both metric (2.1) and $f$ symbols in (2.3) and (2.4). In particular, the first and the third relations in (2.6) can be generalized to the spin-2,3,4 currents with the adjoint index $a$ according to the coset (1.1) we are considering.

We can express the stress energy tensor [2], by Sugawara construction,

$$
\begin{aligned}
T(z)= & \frac{1}{2(k+N+M)}\left[J^{\alpha} J^{\alpha}+J^{a} J^{a}+\delta_{\rho \bar{\sigma}} \delta_{j \bar{i}} J^{(\rho \bar{i})} J^{(\bar{\sigma} j)}+\delta_{\rho \bar{\sigma}} \delta_{j \bar{i}} J^{(\bar{\sigma} j)} J^{(\rho \bar{i})}+J^{u(1)} J^{u(1)}\right](z) \\
& -\frac{1}{2(k+N)} J^{\alpha} J^{\alpha}(z)-\frac{1}{2 k} J^{u(1)} J^{u(1)}(z) .
\end{aligned}
$$

The first five terms of (2.7) come from the $\mathrm{SU}(N+M)$ of the coset (1.1) while the remaining ones come from the $\mathrm{SU}(N) \times \mathrm{U}(1)$ of the coset. Note that we can move the $J^{(\rho \bar{i})}$ in the fourth term of (2.7) to the left and combine it with the third term together with a derivative term according to the relation $\delta_{\rho \bar{\sigma}} \delta_{j \bar{i}}\left[J^{(\bar{\sigma} j)}, J^{(\rho \bar{i})}\right]=-M N \sqrt{\frac{M+N}{M N}} \partial J^{u(1)}$ which will be used several times in this paper. Then we have the following OPE

$$
T(z) T(w)=\frac{1}{(z-w)^{4}} \frac{c}{2}+\frac{1}{(z-w)^{2}} 2 T(w)+\frac{1}{(z-w)} \partial T(w)+\cdots .
$$

It is rather nontrivial to check this OPE (2.8) explicitly by using the (2.5). Here the central charge in (2.8) is given by [2]

$$
\begin{aligned}
c & =\frac{k\left((N+M)^{2}-1\right)}{(k+M+N)}-\frac{k\left(N^{2}-1\right)}{(k+N)}-1 \\
& =\frac{\left(-k^{2}+k^{2} M^{2}-2 k N-M N+2 k^{2} M N+k M^{2} N-N^{2}+k M N^{2}\right)}{(k+N)(k+M+N)} .
\end{aligned}
$$

Furthermore, the spin-1 current is primary operator under the stress energy tensor (2.7)

$$
T(z) J^{a}(w)=\frac{1}{(z-w)^{2}} J^{a}(w)+\frac{1}{(z-w)} \partial J^{a}(w)+\cdots .
$$

Note that $T(z)$ is a singlet under the horizontal subalgebra $\mathrm{SU}(M)$ [21]. The OPEs between $T(z)$ and $J^{\alpha}(w)$ (and $J^{u(1)}(w)$ ) are regular. When we further divide the $\mathrm{SU}(M)$ piece in the coset (1.1) and subtract the corresponding stress energy tensor, $\frac{1}{2(k+M)} J^{a} J^{a}(w)$, from (2.7), then this modified stress energy tensor is no longer singular OPE with spin-1 current $J^{a}(w)$.

\subsection{A charged spin 2 current}

The next question is whether the spin-2 current transforming as adjoint representation of $\mathrm{SU}(M)$ exists or not. If there exists, then how do we construct explicitly? It is natural to require that it should transform as a primary operator under the stress energy tensor (2.7). The nontrivial requirement is the relation between the previous spin- 1 current and this spin-2 current. In general, the second order pole of this OPE contains the spin-1 current with two free adjoint indices while the first order pole contains the composite spin- 2 operators contracted with the appropriate indices. In the specific basis, the spin- 2 current 
can transform as the "primary" operator under the spin-1 current [22]. Furthermore, the spin-2 current should transform under the adjoint representation of the horizontal finite dimensional Lie algebra $\mathrm{SU}(M)[21]$.

Among five spin-1 currents, we can make the quadratic terms between them with derivative terms in order to have spin-2 operator. The nontrivial term is given by the $\mathrm{SU}(M)$ generator multiplied by the spin-1 current transforming as $(\mathbf{N}, \overline{\mathbf{M}})$ and its conjugated one. Moreover, the fundamental and antifundamental indices of $\mathrm{SU}(N)$ should be contracted each other. We expect that there should be the spin- 2 operator contracted by $d$ symbol $[23,24]$ from the adjoint spin-1 current $J^{a}(z)$. It turns out that a charged spin-2 current [2] is given by ${ }^{4}$

$$
\begin{aligned}
K^{a}(z)= & \delta_{\rho \bar{\sigma}} t_{j \bar{i}}^{a}\left(J^{(\overline{\bar{i}})} J^{(\bar{\sigma} j)}+J^{(\bar{\sigma} j)} J^{(\bar{\rho} \bar{i})}\right)(z)-\frac{N}{(M+2 k)} d^{a b c} J^{b} J^{c}(z) \\
& +\frac{2 N}{k} \sqrt{\frac{M+N}{M N}} J^{a} J^{u(1)}(z) .
\end{aligned}
$$

Note that the third term of $(2.11)$ occurs in $[23,24]$. Instead of introducing the arbitrary coefficients, we will check whether the above result is consistent with other conditions.

Now we can compute the OPE between $J^{u(1)}(z)$ and $K^{a}(w)$ and it turns out that the second order pole of this OPE coming from the first two and last terms of (2.11) has $J^{a}(w)$ term whose coefficient vanishes, similar to the third one of (2.6). Moreover, the OPE between $J^{\alpha}(z)$ and $K^{a}(w)$ can be obtained from the first two terms of (2.11) and this leads to the vanishing of this OPE, along the line of the first relation of (2.6), where the traceless conditions for the generators $t_{\rho \bar{\sigma}}^{\alpha}$ and $t_{i \bar{j}}^{a}$ are used. From the OPE between $J^{a}(z)$ and $K^{b}(w)$, the second order pole vanishes by using the identity that the triple product $d f f$ is proportional to $d$ symbol [23-25]. We also consider $\partial J^{a}(z)$ term in $(2.11)$ but the vanishing of third order pole of the OPE between $J^{a}(z)$ and $K^{b}(w)$ does not allow us to add this term. Finally, the first order pole of this OPE can be written in terms of $i f^{a b}{ }_{c} K^{c}(w)$.

Therefore, we summarize that the charged spin- 2 current has the following OPE

$$
J^{a}(z) K^{b}(w)=\frac{1}{(z-w)} i f_{c}^{a b} K^{c}(w)+\cdots
$$

We can compute the commutator $\left[J_{0}^{a}, K^{b}(w)\right]$ and this leads to $i f^{a b c} K^{c}(w)$ from the result of (2.12). In other words, the spin-2 current transforms under the adjoint representation of the horizontal finite dimensional Lie algebra $\mathrm{SU}(M)$ as mentioned before. Here $J_{0}^{a}$ is the Laurent zero mode of spin-1 current $J^{a}(z)$ [21]. Because the complete expression of this charged spin-2 current is given by (2.11), we can calculate the OPE with the stress energy tensor (2.7) and it is given by

$$
T(z) K^{a}(w)=\frac{1}{(z-w)^{2}} 2 K^{a}(w)+\frac{1}{(z-w)} \partial K^{a}(w)+\cdots
$$

\footnotetext{
${ }^{4}$ We have the relation $\delta_{\rho \bar{\sigma}} t_{j \bar{i}}^{a} J^{(\bar{\sigma} j)} J^{(\rho \bar{i})}(z)=\delta_{\rho \bar{\sigma}} t_{j \bar{i}}^{a} J^{(\rho \bar{i})} J^{(\bar{\sigma} j)}(z)+N \partial J^{a}(z)$ from the last OPE of (2.5) with the help of [23].
} 
where the relation (2.10) and other ones are used. So far, the currents are given by the stress energy tensor (2.7), the spin-1 current and the spin-2 current (2.11). Their OPEs are given by $(2.8),(2.10),(2.13)$, the fourth relation of $(2.5)$, and (2.12).

\section{$2.2 \quad$ A charged spin 3 current}

We would like to construct the charged spin-3 current as we did in previous subsection. This charged spin-3 current should be a primary operator under the stress energy tensor (2.7). We expect that the cubic term of $\mathrm{SU}(M)$ adjoint spin-1 current with the fourth order $d$ symbols $[24,26]$ as a nonderivative term can arise. For the OPE with the spin-1 current, we require the previous "primary" condition under the spin-1 current.

It turns out that the charged spin-3 current which was obtained by using the works of $[27-29]$ has the following terms ${ }^{5}$

$$
\begin{aligned}
P^{a}(z)= & a_{1} t_{\rho \bar{\sigma}}^{\alpha} t_{j \bar{i}}^{a} J^{\alpha} J^{(\overline{\rho i})} J^{(\bar{\sigma} j)}(z)+a_{2} J^{\alpha} J^{\alpha} J^{a}(z)+a_{3} J^{b} J^{b} J^{a}(z)+a_{4} J^{a} J^{u(1)} J^{u(1)}(z) \\
& +a_{5} d^{a b c} \delta_{\rho \bar{\rho}} t_{j \bar{i}}^{b} J^{c}\left(J^{(\rho \bar{i})} J^{(\bar{\rho} j)}+J^{(\bar{\rho} j)} J^{(\bar{\rho} \bar{i})}\right)(z)+a_{7} \delta_{\rho \bar{\sigma}} t_{j \bar{i}}^{a} J^{u(1)}\left(J^{(\rho \bar{i})} J^{(\bar{\sigma} j)}+J^{(\bar{\sigma} j)} J^{(\overline{\bar{i}})}\right)(z) \\
& +a_{8} \delta_{\rho \bar{\sigma}} \delta_{j \bar{i}} J^{a}\left(J^{(\rho \bar{i})} J^{(\bar{\sigma} j)}+J^{(\bar{\sigma} j)} J^{(\rho \bar{i})}\right)(z)+a_{9} d^{a b c} J^{b} J^{c} J^{u(1)}(z)+a_{11} i f^{a b c} \partial J^{b} J^{c}(z) \\
& +a_{12} \delta_{\rho \bar{\sigma}} t_{j \bar{i}}^{a} \partial J^{(\rho \bar{i})} J^{(\bar{\sigma} j)}(z)+a_{13} \delta_{\rho \bar{\sigma}} t_{j \bar{i}}^{a} \partial J^{(\bar{\sigma} j)} J^{(\rho \bar{i})}(z)+a_{16} \partial^{2} J^{a}(z) \\
& +a_{17} 6 \operatorname{Tr}\left(t^{a} t^{(b} t^{c} t^{d)}\right) J^{b} J^{c} J^{d}(z) .
\end{aligned}
$$

The $a_{3}, a_{11}, a_{16}$ and $a_{17}$ terms contain the spin- 1 current only. ${ }^{6}$ The $a_{17}$ term is related to the above cubic term with the fourth order $d$ symbols mentioned before. We understand the $a_{2}, a_{4}$ and $a_{9}$ terms because the indices except the free adjoint index $a$ are contracted properly. The nontrivial parts are given by the remaining six terms. The free index $a$ arises in the generator $t_{j \bar{i}}^{a}$, the spin-1 current $J^{a}$ and the $d^{a b c}$ symbols. They contain the spin-1 currents transforming as $(\mathbf{N}, \overline{\mathbf{M}})$ or $(\overline{\mathbf{N}}, \mathbf{M})$. For $a_{5}, a_{7}, a_{12}$ and $a_{13}$ terms, the Kronecker delta symbols are multiplied in order to contract with the fundamental and antifundamental indices of $\mathrm{SU}(N)$ each other. For $a_{8}$ term, there exists further Kronecker delta symbols associated with the fundamental and antifundamental indices of $\mathrm{SU}(M)$. Note that in the $a_{1}$ term, there is an additional generator $t_{\rho \bar{\sigma}}^{\alpha}$ contracted with three other indices. We do not get the $a_{1}$ term from the each term of charged spin- 2 current and other operators. Therefore, the $a_{1}$ term is crucial for the construction of an independent charged spin- 3 current.

Now we would like to construct the spin-3 current step by step explicitly by assuming the operator contents of [2]. We calculate the OPEs by hand without using the method given in [2] where they have obtained this charged spin-3 current for several fixed low $(N, M)$ values and extracted the $(N, M)$ dependence of relative coefficients as well as $k$ dependence. By requiring that we should have the condition $J^{\alpha}(z) P^{a}(w)=0$, along the line of the first relation of (2.6), where the corresponding terms in (2.14) are given by

\footnotetext{
${ }^{5}$ The coefficients $a_{6}, a_{10}, a_{14}$ and $a_{15}$ are vanishing where the corresponding terms are given by $a_{6} i f^{a b c} \delta_{\rho \bar{\rho}} t_{j \bar{i}}^{b} J^{c}\left(J^{(\rho \bar{i})} J^{(\bar{\rho} j)}++J^{(\bar{\rho} j)} J^{(\rho \bar{i})}\right)(z)+a_{10} d^{a b c} \partial J^{b} J^{c}(z)+a_{14} J^{a} \partial J^{u(1)}(z)+a_{15} \partial J^{a} J^{u(1)}(z)$. In order to check (2.14) we keep these terms also.

${ }^{6}$ The $a_{17}$ term can be written as $\frac{6}{M} J^{a} J^{b} J^{b}+\frac{3}{2}(i f+d)^{a b e} d^{e c d} J^{b} J^{c} J^{d}+\frac{3}{2}(i f+d)^{a d e} d^{e b c} i f^{b d f} \partial J^{f} J^{c}-$ $\frac{1}{4}(i f+d)^{a d e} f^{c d f} f^{b f g} \partial^{2} J^{g}+\frac{6}{M} i f^{b a c} \partial J^{c} J^{b}-\frac{1}{M} f^{b a c} f^{b c d} \partial^{2} J^{d}$.
} 
$a_{1}, a_{2}, a_{8}, a_{12}$ and $a_{13}$ terms, the second order pole provides the following equations

$$
\begin{array}{r}
{\left[(2 N+k) a_{1}+\left(a_{12}-a_{13}\right)\right] t_{\rho \bar{\sigma}}^{\alpha} t_{j \bar{i}}^{a} J^{(\rho \bar{i})} J^{(\bar{\sigma} j)}(w)=0} \\
{\left[a_{1}-2(N+k) a_{2}-2 M a_{8}\right] J^{\alpha} J^{a}(w)=0 .}
\end{array}
$$

Here the relation $\left[t^{\alpha}, t^{\beta}\right]=i f_{\gamma}^{\alpha \beta} t^{\gamma}$ is used in (2.15). Moreover, from (2.5), we have the following identity (See also [23])

$$
\left[J^{(\rho \bar{i})}, J^{(\bar{\sigma} j)}\right]=i f_{u(1)}^{(\rho \bar{i})(\bar{\sigma} j)} \partial J^{u(1)}+i f^{(\bar{\rho} \bar{i})(\bar{\sigma} j)} \partial J^{\alpha}+i f^{(\rho \bar{i})(\bar{\sigma} j)}{ }_{a} \partial J^{a} .
$$

Then the contribution from the second term of $a_{8}$ in (2.14) is the same as the one from the first term because the additional two delta symbols in $a_{8}$ term can act on (2.16) which leads to zero value.

Similarly, the regularity condition $J^{u(1)}(z) P^{a}(w)=0$, similar to the third relation of (2.6), gives the following equations we should have

$$
\begin{array}{r}
{\left[-N \sqrt{\frac{M+N}{M N}} a_{12}+2 k a_{14}-N \sqrt{\frac{M+N}{M N}} a_{13}\right] J^{a}(w)=0,} \\
{\left[2 k a_{4}-2 N \sqrt{\frac{M+N}{M N}} a_{7}+2(M+N) a_{8}\right] J^{a} J^{u(1)}(w)=0,} \\
{\left[-2 N \sqrt{\frac{M+N}{M N}} a_{5}+k a_{9}\right] d^{a b c} J^{b} J^{c}(w)=0,} \\
{\left[2 k a_{7}+\sqrt{\frac{M+N}{M N}} a_{12}-\sqrt{\frac{M+N}{M N}} a_{13}\right] \delta_{\rho \bar{\sigma}} t_{j \bar{i}}^{a} J^{(\rho \bar{i})} J^{(\bar{\sigma} j)}(w)=0,} \\
{\left[-2 M N \sqrt{\frac{M+N}{M N}} a_{6}+k N a_{7}-N \sqrt{\frac{M+N}{M N}} a_{13}+k a_{15}\right] \partial J^{a}(w)=0 .}
\end{array}
$$

Each term of the last four terms can be seen from the charged spin-2 current in (2.11). In the last relation of (2.17), the identity $f_{c}^{a b} J^{b} J^{c}(w)=i M \partial J^{a}(w)[23,24]$ is used. In the computation of $a_{7}$ term, there exists the relation $\delta_{\rho \bar{\sigma}} t_{j \bar{i}}^{a} J^{(\bar{\sigma} j)} J^{(\rho \bar{i})}(w)=\delta_{\rho \bar{\sigma}} t_{j \bar{i}}^{a} J^{(\rho \bar{i})} J^{(\bar{\sigma} j)}(w)+$ $N \partial J^{a}(w)$ which can be obtained from the relation (2.16).

Let us consider the OPE between $J^{a}(z)$ and $P^{b}(w)$. The fourth and third order poles of this OPE give us

$$
\begin{gathered}
{\left[2 M k a_{3}-2 M k a_{11}-k N a_{12}+k N a_{13}+6 k a_{16}\right] \delta^{a b}=0} \\
{\left[2(M-k) a_{3}+\left(M^{2}-4\right) \frac{N}{M} a_{5}+2 N a_{8}-(2 k+M) a_{11}-\frac{N}{2} a_{12}\right.} \\
\left.+\frac{N}{2} a_{13}+2 a_{16}+\left(M^{2}+6\right) a_{17}\right] i f_{c}^{a b} J^{c}(w)=0 \\
{\left[4 N M \sqrt{\frac{M+N}{M N}} a_{6}-N \sqrt{\frac{M+N}{M N}} a_{12}-N \sqrt{\frac{M+N}{M N}} a_{13}+2 k a_{15}\right] \delta^{a b} J^{u(1)}(w)=0} \\
{\left[-N M a_{6}+(2 k+M) a_{10}+\frac{N}{2} a_{12}+\frac{N}{2} a_{13}\right] d^{a b c} J^{c}(w)=0 .}
\end{gathered}
$$


In the calculation of the second relation of (2.18), we use the following relation

$$
\operatorname{Tr}\left(t^{i} t^{b} t^{c} t^{d}\right)=\frac{1}{M} \delta^{i b} \delta^{c d}+\frac{1}{4}(i f+d)^{i b e}(i f+d)^{e c d} .
$$

This can be obtained by recalling the fact that the product of two generators can be written in terms of Kronecker delta symbol with identity matrix, $f$ and $d$ symbols with generator and we can multiply further generators successively. By multiplying three $f$ symbols into (2.19), we obtain the intermediate result

$$
\begin{aligned}
& \operatorname{Tr}\left(t^{i} t^{b} t^{c} t^{d}\right) f^{a b f} f^{f c g} f^{g d h}= \\
& \quad-2 f^{a i h}+\frac{1}{4}\left[M^{2} f^{i a h}-i M^{2} d^{h a i}-i M^{2} d^{i a h}-\left(M^{2}-4\right) f^{i h a}\right],
\end{aligned}
$$

where the identities for the triple products $f f f$ and $d f f[23,24]$ are used in $(2.20)$. Then the remaining five similar terms can be obtained and by adding these we arrive at the final contribution $\left(M^{2}+6\right) a_{17} i f^{a b c} J^{c}(w)$ in (2.18).

Let us describe the second order pole which will be more complicated. We have the following result

$$
\begin{aligned}
& {\left[-a_{1}+k a_{2}\right] \delta^{a b} J^{\alpha} J^{\alpha}(w)=0, \quad\left[k a_{4}-2 N \sqrt{\frac{M+N}{M N}} a_{7}\right] \delta^{a b} J^{u(1)} J^{u(1)}(w)=0,} \\
& {\left[-2 N \sqrt{\frac{M+N}{M N}} a_{5}+N a_{7}+(2 k+M) a_{9}\right] d^{a b c} J^{c} J^{u(1)}(w)=0,} \\
& {\left[-2 N \sqrt{\frac{M+N}{M N}} a_{6}+a_{15}\right] i f^{a b c} J^{c} J^{u(1)}(w)=0,} \\
& {\left[k a_{14}-k N M \sqrt{\frac{M+N}{M N}} a_{8}-N \sqrt{\frac{M+N}{M N}} a_{13}\right] \delta^{a b} \partial J^{u(1)}(w)=0,} \\
& {\left[2(k+M) a_{5}-\frac{1}{2} a_{12}+\frac{1}{2} a_{13}\right] d^{a b c} \delta_{\rho \bar{\sigma}} t_{j \bar{i}}^{c} J^{(\rho \bar{i})} J^{(\bar{\sigma} j)}(w)=0,} \\
& {\left[-2(k+M) a_{6}+\frac{1}{2} a_{12}+\frac{1}{2} a_{13}\right] i f^{a b c} \delta_{\rho \bar{\sigma}} t_{j \bar{i}}^{c} J^{(\bar{\rho} \bar{i})} J^{(\bar{\sigma} j)}(w)=0,} \\
& {\left[2 k a_{8}-\frac{1}{M} a_{12}+\frac{1}{M} a_{13}\right] \delta^{a b} \delta_{\rho \bar{\sigma}} \delta_{j \bar{i}} J^{(\bar{\rho} \bar{i})} J^{(\bar{\sigma} j)}(w)=0,} \\
& {\left[-N a_{6}+a_{10}\right] i f^{a c e} d^{b c d} J^{e} J^{d}(w)=0,} \\
& {\left[k a_{10}+N(k+M) a_{5}+\frac{N}{2} a_{13}+M N a_{6}\right] d^{a b c} \partial J^{c}(w)=0,} \\
& {\left[-2 a_{3}-a_{11}+\frac{3}{2} k a_{17}\right] d^{a b e} d^{e c d} J^{c} J^{d}(w)=0,}
\end{aligned}
$$




$$
\begin{aligned}
& {\left[2(k+M) a_{3}+2 N a_{8}+\frac{4}{M}\left(2 a_{3}+a_{11}\right)+\left(\frac{12 k}{M}+18\right) a_{17}\right] J^{a} J^{b}(w)=0,} \\
& {\left[k a_{3}-\frac{4}{M}\left(2 a_{3}+a_{11}\right)+\left(\frac{6 k}{M}-6\right) a_{17}\right] \delta^{a b} J^{c} J^{c}(w)=0} \\
& {\left[N a_{5}+\left(2 a_{3}+a_{11}\right)+3(k+M) a_{17}\right] d^{a c d} d^{b c e} J^{d} J^{e}(w)=0} \\
& {\left[k a_{11}+2 a_{16}+\frac{N}{2} a_{13}-N(k+M) a_{6}-\frac{N}{M}\left(M^{2}-4\right) a_{5}-\frac{1}{M}\left(M^{2}-4\right)\left(2 a_{3}+a_{11}\right)\right.} \\
& \left.\quad+\left(-\frac{3}{2} k M+\left(-2 M^{2}-6\right)\right) a_{17}-2 N a_{8}-\frac{4}{M}\left(2 a_{3}+a_{11}\right)\right] i f^{a b c} \partial J^{c}(w)=0
\end{aligned}
$$

We rewrite the term $f^{a c d} f^{d b e} J^{c} J^{e}(w)$ in terms of Kronecker delta $\delta$ and $d$ symbols by using the corresponding identity [21, 24]. For the calculation of last five relations associated with $a_{17}$ term in (2.21), the identities containing the quartic products of $f f f f, f f f d$ and $f f d d[24,25]$ are used. Note that although there are also $f^{a e c} d^{b e d} J^{c} J^{d}(w)$ and $d^{a b c} \partial J^{c}(w)$ in general, those contributions from the coefficient $a_{17}$ become zero.

By solving the above equations (2.15), (2.17), (2.18) and (2.21), we obtain the coefficients appearing in the spin-3 current as follows:

$$
\begin{aligned}
& a_{2}=\frac{a_{1}}{k}, \\
& a_{3}=\frac{N(k+2 N)}{k(k+M)(3 k+2 M)} a_{1}, \quad a_{4}=\frac{(k+2 N)(M+N)}{k^{2} M} a_{1}, \\
& a_{5}=-\frac{(k+2 N)}{4(k+M)} a_{1}, \quad a_{7}=\frac{(k+2 N)}{2 k} \sqrt{\frac{M+N}{M N}} a_{1}, \quad a_{8}=-\frac{(k+2 N)}{2 k M} a_{1}, \\
& a_{9}=-\frac{(k+2 N) N}{2 k(k+M)} \sqrt{\frac{(M+N)}{M N}} a_{1}, \quad a_{11}=\frac{\left(k^{2}-8\right) N(k+2 N)}{4 k(k+M)(3 k+2 M)} a_{1}, \\
& a_{12}=-\frac{1}{2}(k+2 N) a_{1}, \quad a_{13}=\frac{1}{2}(k+2 N) a_{1}, \\
& a_{16}=-\frac{N\left(6 k^{3}+9 k^{2} M+4 k M^{2}+12 M\right)(k+2 N)}{12 k(k+M)(3 k+2 M)} a_{1}, \quad a_{17}=\frac{N(k+2 N)}{6(k+M)(3 k+2 M)} a_{1} \text {. }
\end{aligned}
$$

Except the coefficient $a_{2}$, all the coefficients contain the factor $(k+2 N)$. These are the same as the ones in [2]. As described in the footnote 5 , the four coefficients, $a_{6}, a_{10}, a_{14}$ and $a_{15}$ are vanishing.

Also we have the primary condition under the stress energy tensor mentioned before

$$
T(z) P^{a}(w)=\frac{1}{(z-w)^{2}} 3 P^{a}(w)+\frac{1}{(z-w)} \partial P^{a}(w)+\cdots
$$

In order to check this condition (2.23), the relations (2.10) can be used.

After using the vanishing of the fourth, third and second order poles we are left with the first order pole and can be written as

$$
J^{a}(z) P^{b}(w)=\frac{1}{(z-w)} i f^{a b c} P^{c}(w)+\cdots
$$


where the fundamental relations (2.5) can be used in (2.24). As explained in (2.12), the spin-3 current transforms under the adjoint representation of $\mathrm{SU}(M)$. Once again, the charged spin-3 current is primary operator via (2.23) and (2.24).

\subsection{An uncharged spin 3 current}

How do we construct the higher spin-3 current which is neutral under the spin- 1 current? We should write down the possible composite spin- 3 operators and determine the relative coefficients by imposing the basic conditions coming from the coset (1.1). As explained before, we should require that this spin-3 current transforms as the primary operator under the stress energy tensor (2.7).

It turns out that the uncharged spin-3 current [2] has the following independent terms ${ }^{7}$

$$
\begin{aligned}
W^{(3)}(z)= & b_{1} d^{\alpha \beta \gamma} J^{\alpha} J^{\beta} J^{\gamma}(z)+b_{2} d^{a b c} J^{a} J^{b} J^{c}(z)+b_{3} J^{u(1)} J^{u(1)} J^{u(1)}(z)+b_{4} J^{\alpha} J^{\alpha} J^{u(1)}(z) \\
& +b_{5} J^{a} J^{a} J^{u(1)}(z)+b_{6} t_{\rho \bar{\sigma}}^{\alpha} \delta_{j \bar{i}} J^{\alpha}\left(J^{(\rho \bar{i})} J^{(\bar{\sigma} j)}+J^{(\bar{\sigma} j)} J^{(\bar{\rho} \bar{i})}\right)(z) \\
& +b_{7} \delta_{\rho \bar{\sigma}} t_{j \bar{i}}^{a} J^{a}\left(J^{(\bar{\rho} \bar{i})} J^{(\bar{\sigma} j)}+J^{(\bar{\sigma} j)} J^{(\overline{\bar{i}})}\right)(z)+b_{8} \delta_{\rho \bar{\sigma}} \delta_{j \bar{i}} J^{u(1)}\left(J^{(\rho \bar{i})} J^{(\bar{\sigma} j)}+J^{(\bar{\sigma} j)} J^{(\bar{\rho} \bar{i})}\right)(z) \\
& +b_{12} \delta_{\rho \bar{\sigma}} \delta_{j \bar{i}} \partial J^{(\rho \bar{i})} J^{(\bar{\sigma} j)}(z)+b_{13} \delta_{\rho \bar{\sigma}} \delta_{j \bar{i}} \partial J^{(\bar{\sigma} j)} J^{(\rho \bar{i})}(z)+b_{14} \partial^{2} J^{u(1)}(z) .
\end{aligned}
$$

The second term can be seen from the work of [23]. The $b_{2}, b_{5}$ and $b_{7}$ terms can be seen from the terms of spin-2 current in (2.11). When we differentiate the stress energy tensor (2.7), then we observe the $b_{12}$ and $b_{13}$ terms. For the $b_{6}$ term, we have seen similar $a_{1}$ term in the charged spin-3 current.

The regularity condition $J^{\alpha}(z) W(w)=0$ implies the following relations coming from the third and second order poles

$$
\begin{array}{r}
{\left[2(k+N) b_{9}+M b_{12}+M b_{13}\right] J^{\alpha}(w)=0,} \\
{\left[3(k+N) b_{1}+M b_{6}\right] d^{\alpha \beta \gamma} J^{\beta} J^{\gamma}(w)=0,} \\
{\left[2(k+N) b_{4}+2 M b_{8}+2 M \sqrt{\frac{M+N}{M N}} b_{6}\right] J^{\alpha} J^{u(1)}(w)=0,} \\
{\left[(k+N) b_{9}+M b_{13}-(k M+2 M N) b_{6}\right] \partial J^{\alpha}(w)=0,} \\
{\left[2(k+2 N) b_{6}+b_{12}-b_{13}\right] \delta_{j \bar{i}} t_{\rho \bar{\sigma}}^{\alpha} J^{(\rho \bar{i})} J^{(\bar{\sigma} j)}(w)=0 .}
\end{array}
$$

In this calculation, we have the identities $f^{\alpha \beta \gamma} J^{\beta} J^{\gamma}(w)=i N \partial J^{\alpha}(w)$ and $\operatorname{Tr}\left(t^{\alpha} t^{\beta} t^{\gamma}\right)=$ $\frac{1}{2}(i f+d)^{\alpha \beta \gamma}$ as described before.

\footnotetext{
${ }^{7}$ The coefficients $b_{9}, b_{10}$ and $b_{11}$ are vanishing and the corresponding terms are given by $b_{9} \partial J^{\alpha} J^{\alpha}(z)+$ $b_{10} \partial J^{a} J^{a}(z)+b_{11} \partial J^{u(1)} J^{u(1)}(z)$.
} 
Similarly, from the OPE between $J^{u(1)}(z)$ and $W(w)$, we have the following relations from the fourth, the third and the second order poles

$$
\begin{aligned}
& {\left[k M N \sqrt{\frac{M+N}{M N}} b_{12}-k M N \sqrt{\frac{M+N}{M N}} b_{13}+6 k b_{14}\right]=0,} \\
& {\left[2 k b_{11}+(M+N) b_{12}+(M+N) b_{13}\right] J^{u(1)}(w)=0,} \\
& {\left[k b_{4}+2 M \sqrt{\frac{M+N}{M N}} b_{6}\right] J^{\alpha} J^{\alpha}(w)=0, \quad\left[k b_{5}-2 N \sqrt{\frac{M+N}{M N}} b_{7}\right] J^{a} J^{a}(w)=0,} \\
& {\left[2(M+N) b_{8}+3 k b_{3}\right] J^{u(1)} J^{u(1)}(w)=0,} \\
& {\left[2 k b_{8}+\sqrt{\frac{M+N}{M N}} b_{12}-\sqrt{\frac{M+N}{M N}} b_{13}\right] \delta_{\rho \bar{\sigma}} \delta_{j \bar{i}} J^{(\rho \bar{i})} J^{(\bar{\sigma} j)}(w)=0,} \\
& {\left[k b_{11}+k M N \sqrt{\frac{M+N}{M N}} b_{8}-(M+N) b_{13}\right] \partial J^{u(1)}(w)=0 .}
\end{aligned}
$$

The identity $\delta_{\rho \bar{\sigma}} \delta_{j \bar{i}}\left[J^{(\rho \bar{i})}, J^{(\bar{\sigma} j)}\right](w)=\sqrt{\frac{M+N}{M N}} M N \partial J^{u(1)}(w)$ coming from (2.16) is used in the calculation of last two equations of (2.27). If we use the relations (2.26) and (2.27) only, then the coefficients are not determined completely.

In order to calculate the OPE between $T(z)$ and $W(w)$, we should obtain the following nontrivial OPEs

$$
\begin{aligned}
T(z) J^{(\rho \bar{i})}(w)= & \frac{1}{(z-w)^{2}}\left[\frac{\left(-k-M+2 k^{2} M-N+k M N\right)}{2 k M(k+N)}\right] J^{(\rho \bar{i})}(w) \\
& +\frac{1}{(z-w)}\left[\frac{1}{(k+N)} i f_{(\sigma \bar{k})}^{((\bar{\rho}))} J^{\alpha} J^{(\sigma \bar{k})}+\frac{1}{k} i f_{(\sigma \bar{j})}^{(\bar{i}) u(1)} J^{u(1)} J^{(\sigma \bar{j})}\right. \\
& \left.+\partial J^{(\bar{\rho} \bar{i})}\right](w)+\cdots, \\
T(z) J^{(\bar{\rho} j)}(w)= & \frac{1}{(z-w)^{2}}\left[\frac{\left(-k-M+2 k^{2} M-N+k M N\right)}{2 k M(k+N)}\right] J_{(\bar{\rho} j)}^{(w)} \\
& +\frac{1}{(z-w)}\left[\frac{1}{(k+N)} i f_{(\bar{\sigma} k)}^{(\bar{\rho} j) \alpha} J^{\alpha} J^{(\bar{\sigma} k)}+\frac{1}{k} i f_{(\bar{\rho} j) u(1)}^{(\bar{\sigma} k)} J^{u(1)} J^{(\bar{\sigma} k)}\right. \\
& \left.+\partial J^{(\bar{\rho} j)}\right](w)+\cdots
\end{aligned}
$$

In the first order term of (2.28), there exist nontrivial nonlinear terms. Even the second order term has nontrivial coefficients which depend on $N, M$ and $k$ explicitly. In this calculation we use the following identity

$$
t_{\rho_{1} \bar{\sigma}_{1}}^{\alpha} t_{\rho_{2} \bar{\sigma}_{2}}^{\alpha}=\delta_{\rho_{1} \bar{\sigma}_{2}} \delta_{\rho_{2} \bar{\sigma}_{1}}-\frac{1}{N} \delta_{\rho_{1} \bar{\sigma}_{1}} \delta_{\rho_{2} \bar{\sigma}_{2}}, \quad t_{i \bar{j}}^{a} t_{k \bar{l}}^{a}=\delta_{i \bar{l}} \delta_{k \bar{j}}-\frac{1}{M} \delta_{i \bar{j}} \delta_{k \bar{l}}
$$


In (2.29), they satisfy for any four indices and similar relations for contracted indices can be obtained from these identities.

We summarize the fifth, fourth and third order poles in the OPE between $T(z)$ and $W^{(3)}(w)$ as follows:

$$
\begin{aligned}
& {\left[2 k\left(M^{2}-1\right) b_{10}+\frac{N\left(-k-M+2 k^{2} M-N+k M N\right)}{(k+N)}\left(b_{12}+b_{13}\right)\right]=0,} \\
& {\left[k\left(M^{2}-1\right) b_{5}-2 N\left(M^{2}-1\right) \sqrt{\frac{M+N}{M N}} b_{7}+\frac{N\left(-k-M+2 k^{2} M-N+k M N\right)}{(k+N)} b_{8}\right.} \\
& \left.\quad+\frac{\left(-k-M+2 k^{2} M-N+k M N\right)}{2 k(k+N)} N \sqrt{\frac{M+N}{M N}}\left(b_{12}-b_{13}\right)\right] J^{u(1)}(w)=0, \\
& 2 b_{10} J^{a} J^{a}(w)+\left(b_{12}+b_{13}\right)\left[2 \delta_{\rho \bar{\sigma}} \delta_{j \bar{i}} J^{(\rho \bar{i})} J^{(\bar{\sigma} j)}-\frac{(M+N)}{k} J^{u(1)} J^{u(1)}\right. \\
& \left.\quad-\frac{M}{(k+N)} J^{\alpha} J^{\alpha}-M N \sqrt{\frac{M+N}{M N}} \partial J^{u(1)}\right](w)=0 .
\end{aligned}
$$

It can be checked that the contribution from the coefficient $b_{6}$ term vanishes by using the various further contractions between the operators appearing in the contributions from the $b_{12}$ or $b_{13}$ term. We have the following primary condition under the stress energy tensor

$$
T(z) W^{(3)}(w)=\frac{1}{(z-w)^{2}} 3 W^{(3)}(w)+\frac{1}{(z-w)^{2}} \partial W^{(3)}(w)+\cdots .
$$

It will be rather complicated to check this by hand explicitly. If we identify some of the factors in the spin-3 current with the previous known currents, then the corresponding computations will be easier.

By solving (2.26), (2.27) and (2.30), we arrive at the following intermediate result for the coefficients

$$
\begin{aligned}
b_{3} & =\frac{2(k+N)(M+N)(k+2 N)}{k^{2} M} \sqrt{\frac{M+N}{M N}} b_{1}, & b_{4} & =\frac{6(k+N)}{k} \sqrt{\frac{M+N}{M N}} b_{1}, \\
b_{5} & =\frac{2 N}{k} \sqrt{\frac{M+N}{M N}} b_{7}, & b_{6} & =-\frac{3(k+N)}{M} b_{1}, \\
b_{8} & =-\frac{3(k+N)(k+2 N)}{k M} \sqrt{\frac{M+N}{M N}} b_{1}, & b_{12} & =\frac{3(k+N)(k+2 N)}{M} b_{1}, \\
b_{13} & =-\frac{3(k+N)(k+2 N)}{M} b_{1}, & b_{14} & =-N(k+N)(k+2 N) \sqrt{\frac{M+N}{M N}} b_{1} .
\end{aligned}
$$

The coefficients are written in terms of $b_{1}$ and $b_{7}$ and moreover the coefficient $b_{2}$ is not determined yet. Except the coefficients of $b_{4}$ and $b_{6}$, all the coefficients contain the factor $(k+2 N)$. We will analyze further in section 7 and determine the remaining coefficients completely. Therefore, we have checked that the expressions for the spin-3 current is correct for any $(N, M)$ and $k$. 


\section{The OPE between the charged higher spin-2 current and itself}

In this section, we would like to construct the OPE $K^{a}(z) K^{b}(w)$ which did not appear in [2] by using the explicit realization in (2.11) with the help of (2.5). What they have observed in [2] is that the above OPE is found by assuming that there exist the spin-1,2 currents as well as the stress energy tensor (2.7). Of course, they have constructed the uncharged spin-3 current which does not appear in the above OPE. Moreover, they have used the Jacobi identities between these currents and the relative coefficients appearing in this OPE depend on $(N, M)$ and $k$ explicitly by collecting some of the results for fixed $(N, M)$ values. Furthermore, their construction does not tell us any information on the coset model.

On the other hand, in our construction we use the explicit realization of coset and the currents are given by $(2.7),(2.11),(2.14)$ and (2.25). We will observe that there exists a charged spin-3 current described in (2.14) in the first order pole of the OPE.

It is useful to calculate the OPEs between $K^{a}(z)$ and other spin-1 operators. We have (2.12) and the OPE between $K^{a}(z)$ and $J^{\alpha}(w)$ and the OPE between $K^{a}(z)$ and $J^{u(1)}(w)$ have trivial results from the analysis of the subsection 2.1. Then the remaining nontrivial OPEs are given by

$$
\begin{aligned}
K^{a}(z) J^{(\rho \bar{i})}(w)= & \frac{1}{(z-w)^{2}}\left[\frac{2\left(k^{2}-1\right)(2 k+M+N)}{k(2 k+M)}\right] \delta^{k \bar{i}} t_{k \bar{j}}^{a} J^{(\rho \bar{j})}(w) \\
& +\frac{1}{(z-w)}\left[2(k+N) \delta_{k \bar{j}}\left(t^{a}\right)^{\bar{i} k} \partial J^{(\rho \bar{j})}-\frac{2(k+N)}{k} \sqrt{\frac{M+N}{M N}} \delta^{k \bar{i}} t_{k \bar{j}}^{a} J^{u(1)} J^{(\rho \bar{j})}\right. \\
& +\frac{2}{k M}(k+M+N) J^{a} J^{(\rho \bar{i})}-\left(i f-\frac{(2 k+M+2 N)}{(2 k+M)} d\right)^{a b c} \delta^{k \bar{i}} t_{k \bar{j}}^{c} J^{b} J^{(\rho \bar{j})} \\
& \left.-2 \delta^{k \bar{i}} \delta_{\sigma \overline{\sigma_{1}}}\left(t^{\alpha}\right)^{\overline{\sigma_{1}} \rho} t_{k \bar{j}}^{a} J^{\alpha} J^{(\sigma \bar{j})}\right](w)+\cdots, \\
K^{a}(z) J^{(\bar{\rho} j)}(w)= & \frac{1}{(z-w)^{2}}\left[\frac{\left.2\left(k^{2}-1\right)(2 k+M+N)\right]}{k(2 k+M)}\right] \delta^{j \bar{l}} t_{k \bar{l}}^{a} J^{(\bar{\rho} k)}(w) \\
& +\frac{1}{(z-w)}\left[2(k+N) \delta_{k \bar{k}_{1}}\left(t^{a}\right)^{\overline{k_{1} j}} \partial J^{(\bar{\rho} k)}+\frac{2(k+N)}{k} \sqrt{\frac{M+N}{M N}} \delta^{j \bar{l}} t_{k \bar{l}}^{a} J^{u(1)} J^{(\bar{\rho} k)}\right. \\
& -\frac{2}{k M}(k+M+N) J^{a} J^{(\bar{\rho} j)}-\left(i f+\frac{(2 k+M+2 N)}{(2 k+M)} d\right)^{a b c} \delta^{j \overline{j_{1}}} t_{k \overline{j_{1}}}^{c} J^{b} J^{(\bar{\rho} k)} \\
& \left.+2 \delta^{j \bar{l}} \delta_{\sigma \overline{\sigma_{1}}}\left(t^{\alpha}\right)^{\bar{\rho} \sigma} t_{k \bar{l}}^{a} J^{\alpha} J^{\left.\left(\overline{\sigma_{1}} k\right)\right]}\right](w)+\cdots
\end{aligned}
$$

These two OPEs look similar but they are different from each other. Based on these OPEs, we can calculate the OPEs between the charged spin- 2 current and the derivative of spin-1 currents by simply taking the derivative with respect to the argument $w$. We use the 
identity of two and triple products of generators

$$
\begin{aligned}
t^{a} t^{b} & =\frac{1}{M} \delta^{a b} \mathbf{1}_{M}+\frac{1}{2}(i f+d)^{a b c} t^{c} \\
t^{a} t^{b} t^{c} & =\frac{1}{M} \delta^{b c} t^{a}+\frac{1}{2 M} \delta^{a d}(i f+d)^{b c d} \mathbf{1}_{M}+\frac{1}{4}(i f+d)^{b c d}(i f+d)^{a d f} t^{f}
\end{aligned}
$$

where the first relation can be obtained from the $f$ and $d$ symbols in (2.3) together with the metric in (2.1) and the second relation can be determined by acting other generator on the first relation.

\subsection{The fourth, third and second order poles}

Then the fourth order pole can be determined by the OPE between the spin- 2 current and the first two terms of spin- 2 current. If we use the property of the footnote 4 , then the contribution from the second term of the spin- 2 current can be expressed as the contribution from the first term and the contribution from the OPE between the spin- 2 current and the derivative of spin- 1 current which can be easily obtained from the defining relation in (2.12).

It turns out that the fourth order pole of this OPE is given by

$$
\left.K^{a}(z) K^{b}(w)\right|_{\frac{1}{(z-w)^{4}}}=\frac{4\left(k^{2}-1\right) N(2 k+M+N)}{(2 k+M)} \delta^{a b},
$$

which is equal to $\frac{c_{1}}{2} \delta^{a b}$ in the notation of [2]. Then we can determine the coefficient

$$
c_{1}=\frac{8\left(k^{2}-1\right) N(2 k+M+N)}{(2 k+M)} .
$$

The free indices $a$ and $b$ arise in the form of invariant Kronecker delta symbols.

How do we obtain the third order pole? By using the trace of triple product of generators appearing in (3.2) leading to the second contribution because the first and last contributions provide zero due to the tracelessness of the generator, the final result can be expressed as a $f$ symbols with spin- 1 current. It turns out that the third order pole of this OPE is given by

$$
\left.K^{a}(z) K^{b}(w)\right|_{\frac{1}{(z-w)^{3}}}=\frac{4\left(k^{2}-1\right) N(2 k+M+N)}{k(2 k+M)} i f^{a b c} J^{c}(w),
$$

which is given by $c_{2} i f^{a b c} J^{c}(w)$ in the notation of [2]. Therefore, we have the coefficient

$$
c_{2}=\frac{4\left(k^{2}-1\right) N(2 k+M+N)}{k(2 k+M)} .
$$


Let us present the final result first. The second order pole can be written as

$$
\begin{aligned}
& K^{a}(z) K^{b}(w) \mid \begin{array}{l}
\frac{1}{(z-w)^{2}} \\
\end{array} \\
& -\frac{4(k+N)(M+N)}{k M} \delta^{a b} J^{u(1)} J^{u(1)}(w)-4 \delta^{a b} J^{\alpha} J^{\alpha}(w)-\frac{4 N}{k M}(k+M+N) J^{a} J^{b}(w) \\
& +2 \sqrt{\frac{M+N}{M N}}\left(-\frac{N^{2}}{k} i f+\frac{N\left(4 k^{2}+2 k M+4 k N+M N\right)}{k(2 k+M)} d\right)^{a b c} J^{c} J^{u(1)}(w) \\
& +N\left(i f-\frac{(k+M+2 N)}{(2 k+M)} d\right)^{a e c}(i f+d)^{d b c} J^{e} J^{d}(w)+\frac{8(k+N)}{M} \delta^{a b} \delta^{\rho \bar{\sigma}} \delta_{j \bar{l}} J^{(\rho \bar{l})} J^{(\bar{\sigma} j)}(w) \\
& -2 N i f^{a b c} \delta^{\rho \bar{\sigma}} t_{j \bar{l}}^{c} J^{(\rho \bar{l})} J^{(\bar{\sigma} j)}(w)+\frac{2\left(4 k^{2}+2 k M+4 k N+M N\right)}{(2 k+M)} d^{a b c} \delta^{\rho \bar{\sigma}} t_{j \bar{l}}^{c} J^{(\rho \bar{l})} J^{(\bar{\sigma} j)}(w) \\
& -4 N(k+N) \sqrt{\frac{M+N}{M N}} \delta^{a b} \partial J^{u(1)}(w)+2 k N i f^{a b c} \partial J^{c}(w) \\
& +\frac{2 k N(2 k+M+2 N)}{(2 k+M)} d^{a b c} \partial J^{c}(w)-\frac{M N}{(2 k+M)} d^{a b c} K^{c}(w)+N i f^{a b c} K^{c}(w) .
\end{aligned}
$$

The contribution from the third term of (2.11) is given by the second term of the last line of (3.7). The last term of (3.7) comes from the expression of the second term having a derivative term of $J^{b}(w)$ in the footnote 4 . Then the remaining expressions come from the first two terms in (2.11). Then the operator contents of (3.7) is the same as the ones in $(2.21)$ as expected.

The next question is how we can write down the above expression (3.7) in terms of previous known currents, spin-1,2 currents as well as the stress energy tensor? Of course, there should be a descendant term originating from the third order pole. This is a simple derivative term of spin-1 current with fixed known coefficient. Moreover, it is obvious that there are stress energy tensor and spin-2 current of spin-2. Now it is clear to simplify (3.7) by comparing it with (2.7) and (2.11).

It is easier to look at the terms of singlet operator without having any group indices first. By identifying $J^{u(1)} J^{u(1)}(w)$ term in both (3.7) and (2.7), we observe that the coefficient of $T(w)$ in the second order pole should be equal to

$$
\frac{8}{M}(k+N)(k+M+N)
$$

by focusing on the first term of (3.7). This is equivalent to $2 \frac{c_{1} a_{1, C H}}{c}$ of [2] with (2.9) and (3.4). Then we can extract the coefficient of $a_{1, C H}$ from (3.8) as follows:

$$
a_{1, C H}=\frac{(2 k+M)\left(-k^{2}+k^{2} M^{2}-2 k N-M N+2 k^{2} M N+k M^{2} N-N^{2}+k M N^{2}\right)}{2\left(k^{2}-1\right) M N(2 k+M+N)} .
$$

Then the structure constant (3.8) appearing in the stress energy tensor of the second order pole is determined. Of course, other terms of the stress energy tensor in the second order pole can be checked. 
Let us move to the other structure constant and the coefficient of $d^{a b c} K^{c}(w)$ is given by

$$
\frac{2 k(2 k+M+2 N)}{(2 k+M)},
$$

which is equal to $2 c_{6}$ in [2]. Note that the contribution (3.10) comes from the $d$ term of the second line of (3.7) and the second term in the last line of (3.7) by focusing on the singlet term of (2.11). Then the coefficient of $c_{6}$ of [2] from (3.10) is given by

$$
c_{6}=\frac{k(2 k+M+2 N)}{(2 k+M)} .
$$

Then the structure constant (3.10) appearing in the spin-2 current of the second order pole is determined.

After subtracting the descendant term, the stress energy tensor term and spin- 2 current term from the second order pole, there exists the sum of some nonzero composite operators which corresponds to a quasi primary operator. We can collect the following nonderivative quadratic $J^{a}$ dependent terms in (3.7)

$$
\begin{aligned}
& \frac{4 N}{M} \delta^{a b} J^{c} J^{c}+\frac{N\left(4 k^{2}+4 k M+M^{2}+M N\right)}{(2 k+M)^{2}} d^{a b e} d^{e c d} J^{c} J^{d} \\
& \quad-\frac{4 N(2 k+M+N)}{k M} J^{a} J^{b}-\frac{2 N(2 k+M+N)}{(2 k+M)} d^{a c e} d^{e b d} J^{c} J^{d} .
\end{aligned}
$$

From the expression of (3.7), it is easy to see that the above terms (3.12) come from the last term of the first line (entering into the third term of (3.12)), the first term of the third line, and the second term of the last line (contributing to the second term of (3.12)) of (3.7). Because we are looking at the particular composite operators, the other terms in (3.7) including the derivative terms should be checked explicitly.

On the other hand, the two invariant fourth order $d$ symbols are studied in [2] as well as the two product of Kronecker delta symbols. Then we can express the above quantities by writing down their invariant tensors in terms of $f$ and $d$ symbols via the first two relations in appendix (A.1). In other words, we have

$$
\begin{aligned}
& {\left[c_{31}+\frac{4}{M} c_{32}-\frac{4}{M} c_{33}+\frac{2 c_{1} a_{1}}{c} \frac{1}{2(k+M+N)}\right] \delta^{a b} J^{c} J^{c}} \\
& \quad+\left[\left(c_{32}-c_{33}\right)-2 c_{6} \frac{N}{(2 k+M)}\right] d^{a b e} d^{e c d} J^{c} J^{d}+\left[\frac{8}{M} c_{33}+c_{34}\right] J^{a} J^{b}+2 c_{33} d^{a c e} d^{e b d} J^{c} J^{d} .
\end{aligned}
$$

Note that these four independent operators appear in (2.21). For the $c_{33}$ term, as we can see in the second relation of appendix (A.1), the various identities can be used. After using the symmetric property of the free indices, then half of them can be rewritten as the other half. It turns out that $f d$ term and the derivative term with $d$ symbols are vanishing.

Then we obtain the following expressions, by using the two equations (3.12) and (3.13),

$$
\begin{aligned}
& c_{31}=-\frac{4\left(4 k^{3}+4 k^{2} M+k M^{2}+8 k^{2} N+6 k M N+M^{2} N+4 k N^{2}+M N^{2}\right)}{M(2 k+M)^{2}}, \\
& c_{32}=\frac{2 k N(2 k+M+N)}{(2 k+M)^{2}}, \quad c_{33}=-\frac{N(2 k+M+N)}{(2 k+M)}, \quad c_{34}=-\frac{4 N(2 k+M+N)}{k(2 k+M)} .
\end{aligned}
$$


Therefore, we have determined the second order pole with (3.9), (3.11) and (3.14) completely. As we emphasized before, the structure constants we have found here are different from the their (3.27) in [2].

\subsection{The first order pole and charged spin-3 current}

Now we can collect all the contributions entering into the first order pole and we arrive at the final results as follows:

$$
\begin{aligned}
& \left.K^{a}(z) K^{b}(w)\right|_{\frac{1}{(z-w)}}=i f^{a b c} N \partial K^{c}(w) \\
& -\frac{N}{(2 k+M)}\left(i f^{a c e} d^{b c d} K^{e} J^{d}+i f^{a d e} d^{b c d} J^{c} K^{e}\right)(w)+\frac{2 N}{k} \sqrt{\frac{M+N}{M N}} i f^{a b c} K^{c} J^{u(1)}(w) \\
& +\delta_{\rho \bar{\sigma}} t_{j \bar{i}}^{b}\left[4(k+N) \delta_{k \bar{l}}\left(t^{a}\right)^{\bar{i} k} \partial J^{(\rho \bar{l})} J^{(\bar{\sigma} j)}-\frac{4}{k}(k+N) \sqrt{\frac{M+N}{M N}} \delta^{k \bar{i}} t_{k \bar{l}}^{a}\left(\left(J^{u(1)} J^{(\rho \bar{l})}\right) J^{(\bar{\sigma} j)}\right)\right. \\
& +\frac{4}{k M}(k+M+N)\left(\left(J^{a} J^{(\rho \bar{i})}\right) J^{(\bar{\sigma} j)}\right) \\
& -2\left(i f-\frac{(2 k+M+2 N)}{(2 k+M)} d\right)^{a c d} \delta^{k \bar{i}} t_{k \bar{l}}^{d}\left(\left(J^{c} J^{(\rho \bar{l})}\right) J^{(\bar{\sigma} j)}\right) \\
& -4 \delta^{k \bar{i}} \delta_{\sigma_{1} \bar{\sigma}_{1}}\left(t^{\alpha}\right)^{\overline{\sigma_{1}} \rho} t_{k \bar{l}}^{a}\left(\left(J^{\alpha} J^{\left(\sigma_{1} \bar{l}\right)}\right) J^{(\bar{\sigma} j)}\right)+4(k+N) \delta_{k \bar{l}}\left(t^{a}\right)^{\bar{l} j} J^{(\rho \bar{i})} \partial J^{(\bar{\sigma} k)} \\
& +\frac{4}{k}(k+N) \sqrt{\frac{M+N}{M N}} \delta^{j \bar{l}} t_{k \bar{l}}^{a} J^{(\rho \bar{i})} J^{u(1)} J^{(\bar{\sigma} k)}-\frac{4}{k M}(k+M+N) J^{(\bar{\rho} \bar{i})} J^{a} J^{(\bar{\sigma} j)} \\
& -2\left(i f+\frac{(2 k+M+2 N)}{(2 k+M)} d\right)^{a c d} \delta^{j \overline{j_{1}}} t_{k \overline{j_{1}}}^{d} J^{(\rho \bar{i})} J^{c} J^{(\bar{\sigma} k)} \\
& \left.+4 \delta^{j \bar{l}} \delta_{\sigma_{1} \bar{\sigma}_{1}}\left(t^{\alpha}\right)^{\bar{\sigma} \sigma_{1}} t_{k \bar{l}}^{a} J^{(\rho \bar{i})} J^{\alpha} J^{\left(\overline{\sigma_{1}} k\right)}\right](w) .
\end{aligned}
$$

Compared to the previous second order pole, it is rather easy to obtain this first order pole because we do not have to consider the additional contractions between the operators. The first two terms in the second line of (3.15) are determined from the OPE between the spin-2 current and the third term of (2.11) while the last term in the second line of (3.15) comes from the OPE between the spin-2 current and the last term of (2.11).

According to the observation of [2], there exist five quasi primary operators including the spin-3 current after subtracting the various descendant operators properly. Let us look at the $J^{\alpha}$ term in (3.15). It appears in the sixth line and the last line. We can easily see that they have the product of two generators and this contains the $f$ symbols with numerical value $\frac{1}{2}$. Then the overall numerical factor will be 4 by adding the above two contributions. Because the operator contents are the same as the one of the first term of spin-3 current (2.14), by extracting the first term of $P^{c}(w)$ in the above first order term (3.15), we determine the structure constant, the coefficient of $P^{c}(w)$ in the right hand side of the OPE

$$
C_{K^{a} K^{b}}^{P^{c}}=\frac{4}{a_{1}} i f^{a b c}
$$


Of course, this is one of the terms among thirteen terms in (2.14). Further analysis on this direction can be done without any difficulty.

Note that the second term of spin-3 current contains only $J^{\alpha}$ and $J^{c}$ term. We can check that this term cannot be seen from (3.15). However, among the list of the five quasi primary operators we mentioned, we can find that term. This implies that we should have exact coefficient in the two places, in the quasi primary operator and the spin- 3 current with opposite signs. Then we can determine the coefficient $a_{3}^{C H}$ in [2] by focusing on the second term of $P^{c}(w)$

$$
i\left[\frac{1}{2(k+M+N)}-\frac{1}{2(k+N)}\right] c_{2} a_{3, C H}+i \frac{4}{a_{1}} a_{2}=0
$$

where (3.16) is used. Note that the two terms inside the bracket in (3.17) are coming from the explicit stress energy tensor in (2.7). From this (3.17) together with (3.6) and (2.22), we have determined the coefficient

$$
a_{3, C H}=\frac{2(2 k+M)(k+N)(k+M+N)}{\left(k^{2}-1\right) M N(2 k+M+N)} .
$$

Then the structure constant appearing in this quasi primary operator is given by the first term of (3.17) with (3.6) and (3.18).

Now we move to the other quasi primary operator. Let us determine the coefficient of $c_{73}$ appearing in the first order pole in [2] by looking at $f^{a b c} d^{c d e} J^{d} J^{e} J^{u(1)}(w)$. Then we have the following relation

$$
-i \frac{4 N^{2}}{k(2 k+M)} \sqrt{\frac{M+N}{M N}}-i \frac{4}{a_{1}} a_{9}-\frac{2 N}{k} \sqrt{\frac{M+N}{M N}} c_{73}=0,
$$

where the first term originates from the second, third and fourth terms of (3.15). In the $c_{73}$ term of (3.19), the relation of third line in appendix (A.1) is used. In the $a_{9}$ term, the relation (3.16) is used. By substituting the value of $a_{9}$ in (2.22) into (3.19), we obtain

$$
c_{73}=i \frac{k(2 k+M+2 N)}{(k+M)(2 k+M)} .
$$

For the $c_{72}$ term having $f^{\text {ace }} d^{\text {bed }}$ in [2], we should focus on the $a_{5}$ term of the spin-3 current $P^{c}(w)$. See also the relations in appendix (A.1). Then we have

$$
-2 i+i \frac{4}{a_{1}} 2 a_{5}+2 c_{73}-2 i c_{72}=0 .
$$

There are two contributions from (3.15) for the first term in (3.21). The corresponding terms are $f$ terms in the fifth and eighth line of (3.15). In the $a_{5}$ term here, the second term of $a_{5}$ appearing in (2.14) can be written in terms of the first term and derivative term. Then the number 2 exists in (3.21). We determine the coefficient $c_{72}$ from (3.21) by using (2.22) and (3.20) as follows:

$$
c_{72}=-\frac{(2 k+M+2 N)}{(2 k+M)} .
$$


Therefore, the structure constant associated with $c_{72}$ and $c_{73}$ terms is completely determined.

Now we consider the quasi primary operator which is cubic terms in the spin- 1 currents. For the coefficient $c_{53}$, we consider $d^{a c f} f^{f b g} d^{g d e} J^{c} J^{d} J^{e}(w)$ term. In this case, we have

$$
i \frac{2 N^{2}}{(2 k+M)^{2}}-3 i c_{53}+2 \frac{N}{(2 k+M)} i c_{72}=0 .
$$

It is rather nontrivial to extract the exact contribution from the $c_{53}$ term with corresponding $d_{52}^{a b c d e}$ tensor. The other contribution from $c_{72}$ can occur here. Therefore, from (3.22) and (3.23), we determine the coefficient $c_{53}$

$$
c_{53}=-\frac{2 N(2 k+M+N)}{3(2 k+M)^{2}} .
$$

By considering the $f^{a b f} d^{f c g} d^{g d e} J^{c} J^{d} J^{e}(w)$ term, we have

$$
i \frac{2 N^{2}}{(2 k+M)^{2}}-i \frac{3}{2} c_{52}-i \frac{3}{2} c_{53}+i \frac{N}{(2 k+M)} c_{72}-i \frac{4}{a_{1}} \frac{3}{2} a_{17}+\frac{N}{(2 k+M)} c_{73}=0 .
$$

Again the first term can be obtained from the first two terms in the second line of (3.15) with Jacobi identity. In this case also, the corresponding invariant tensors associated with $c_{52}$ and $c_{53}$ terms look complicated in appendix (A.1) but if we use the symmetric property of the indices between $c, d$ and $e$ we will obtain simpler expression and we can extract the exact coefficients we presented above. For the $c_{72}$ term, the Jacobi identity is used. It is easy to obtain the coefficient $c_{52}$ by substituting (2.22), (3.24), (3.22) and (3.20) into the above (3.25)

$$
c_{52}=\frac{2 k N(2 k+M+N)}{3(2 k+M)^{2}(3 k+2 M)} .
$$

For the $f^{a b c} J^{c} J^{d} J^{d}(w)$ term, we have

$$
i \frac{4}{a_{1}} a_{3}+i \frac{4}{a_{1}} \frac{6}{M} a_{17}+\frac{1}{2(k+M+N)} c_{2} a_{3, C H}+c_{51}+i \frac{6}{M} c_{52}-i \frac{6}{M} c_{53}=0 .
$$

We can observe the first term with previous structure constant (3.16) in the spin-3 current. It is obvious to see the $a_{51}$ term and we obtain the $c_{52}$ and $c_{53}$ terms with above coefficients. Again, from (2.22), (3.6), (3.18), (3.26) and (3.24), we determine the coefficient $c_{51}$ from $(3.27)$

$$
c_{51}=-i \frac{4\left(6 k^{3}+7 k^{2} M+2 k M^{2}+12 k^{2} N+10 k M N+2 M^{2} N+6 k N^{2}+2 M N^{2}\right)}{k M(2 k+M)(3 k+2 M)} .
$$

We also realize that $a_{2, C H}$ can be obtained from $a_{3, C H}$ in (3.18)

$$
\begin{aligned}
a_{2, C H}= & \frac{1}{6}\left(1-3 a_{3, C H}\right) \\
= & \frac{1}{6\left(k^{2}-1\right) M N(2 k+M+N)}\left(-12 k^{3}-18 k^{2} M-6 k M^{2}-24 k^{2} N-26 k M N\right. \\
& \left.+2 k^{3} M N-7 M^{2} N+k^{2} M^{2} N-12 k N^{2}-7 M N^{2}+k^{2} M N^{2}\right) .
\end{aligned}
$$


Note that this (3.29) is not an independent structure constant because this can be obtained from $a_{3, C H}$. Therefore, we have determined the structure constants with $c_{51}, c_{52}$ and $c_{53}$ terms appearing in the cubic spin- 1 current terms.

We are left with one final quasi primary operator of spin-3 which contains the derivative terms. This is the most nontrivial parts to extract the correct structure constants because the derivative terms appear all over the places. Let us determine the remaining two coefficients, $c_{41}$ and $c_{43}$. For the former, by looking at the $f^{a b c} f^{c d e} \partial J^{d} J^{e}(w)$, we eventually have

$$
\begin{aligned}
& \frac{4}{a_{1}}\left(-2 a_{3}-a_{11}-\frac{3 M}{2} a_{17}\right)+2 c_{41}+i c_{51}-\frac{3 M}{2}\left(c_{52}+c_{53}\right) \\
& \quad+\left(\frac{12}{M}-3 \frac{8-M^{2}}{2 M}\right) c_{53}+\frac{N^{2}}{(2 k+M)}=0 .
\end{aligned}
$$

It is not difficult to check the coefficient for the $c_{51}$ term because it contains already one of the $f$ symbols. For the $c_{52}$ term, we should move the spin- 1 currents to the left in order to obtain the above derivative term with some identity including the $f$ or $d$ symbols. For the $c_{53}$ term, the identity for $f f d d[24,25]$ is used. The last term of $(3.30)$ comes from the fifth line of (3.15) which should be simplified further. Then this will give us the final expression as above. The above (3.30) leads to

$$
\begin{aligned}
c_{41}= & \frac{1}{k M(2 k+M)^{2}(3 k+2 M)}\left(-24 k^{4}-40 k^{3} M-22 k^{2} M^{2}-4 k M^{3}-48 k^{3} N-64 k^{2} M N\right. \\
& +2 k^{4} M N-28 k M^{2} N+3 k^{3} M^{2} N-4 M^{3} N+k^{2} M^{3} N-24 k^{2} N^{2}-20 k M N^{2}+k^{3} M N^{2} \\
& \left.-4 M^{2} N^{2}+k^{2} M^{2} N^{2}\right),
\end{aligned}
$$

where the previous results (2.22), (3.28), (3.26) and (3.24) are used in (3.30).

Now we would like to determine the final undetermined coefficient. For the $c_{43}$ coefficient, we consider the expression of $\left.f^{a b i} K^{a}(z) K^{b}(w)\right|_{\frac{1}{(z-w)}}$. Then we have the relation

$$
\begin{aligned}
- & N \frac{4}{k M}(k+M+N)+N M-N \frac{(2 k+M+2 N)}{(2 k+M)} \frac{\left(M^{2}-4\right)}{M} \\
& -\frac{4}{a_{1}} i 2 M\left[2 i a_{3}+i a_{11}+i\left(\frac{4}{M}+\frac{2}{M}+\frac{\left(M^{2}-4\right)}{M}+\frac{\left(M^{2}-4\right)}{2 M}\right) a_{17}\right] \\
& -2\left(2 M c_{41}+c_{43}\right)-2 M i c_{51}-\left(-2 M \frac{6}{M}-\frac{3}{2} 2 M \frac{\left(M^{2}-4\right)}{M}\right)\left(c_{52}+c_{53}\right) \\
& -\left(2 M \frac{12}{M}+3 M \frac{\left(M^{2}-4\right)}{M}\right) c_{53}=0 .
\end{aligned}
$$

The fourth line of (3.15) contributes the first term of (3.32) if we further simplify nonstandard normal ordering product in the composite operator. Again the fifth line of (3.15) can be simplified and we can check the contribution from this will be the remaining two terms 
in the first line of (3.32). Now we can move to the next line. For the $a_{3}$ term, we obtain the above factor by moving the spin- 1 current to the left. For the $a_{11}$ term, we will have $f f$ term which is proportional to $2 M$. We collect all the contributions from the $a_{17}$ term. From the above (3.32) by substituting (2.22), (3.31), (3.28), (3.26) and (3.24), we arrive at

$$
c_{43}=-\frac{2 M N(2 k+M+N)}{k(2 k+M)^{2}} .
$$

Therefore, we have determined all the structure constants associated with $c_{41}$ and $c_{43}$ appearing in the first order pole. ${ }^{8}$

\subsection{The final OPE}

After collecting the previous results (3.3), (3.5), (3.7) and (3.15), we summarize the OPE, in the notation of [2], between the charged spin-2 current and itself as follows:

$$
\begin{aligned}
K^{a}(z) K^{b}(w)= & \frac{1}{(z-w)^{4}} \frac{c_{1}}{2}+\frac{1}{(z-w)^{3}} i c_{2} f^{a b c} J^{c}(w) \\
& +\frac{1}{(z-w)^{2}}\left[\frac{1}{2} i c_{2} f^{a b c} \partial J^{c}+\frac{2 a_{1, C H} c_{1}}{c} \delta^{a b} T+2 c_{6} d^{a b c} K^{c}\right. \\
& \left.+\left(c_{31} \delta^{a b} \delta^{c d}+c_{32} d_{4 S S 1}^{a b c d}+c_{33} d_{4 S S 2}^{a b c d}+c_{34} \delta^{a c} \delta^{b d}\right) \frac{1}{2}\left(J^{c} J^{d}+J^{d} J^{c}\right)\right](w) \\
& +\frac{1}{(z-w)}\left[\frac{1}{6} i c_{2} f^{a b c} \partial^{2} J^{c}+\frac{1}{2} \partial\left(\frac{2 a_{1, C H} c_{1}}{c} \delta^{a b} T+2 c_{6} d^{a b c} K^{c}\right.\right. \\
& \left.+\left(c_{31} \delta^{a b} \delta^{c d}+c_{32} d_{4 S S 1}^{a b c d}+c_{33} d_{4 S S 2}^{a b c d}+c_{34} \delta^{a c} \delta^{b d}\right) \frac{1}{2}\left(J^{c} J^{d}+J^{d} J^{c}\right)\right) \\
& +i c_{2} a_{3, C H} f^{a b c}\left(T J^{c}-\frac{1}{2} \partial^{2} J^{c}\right) \\
& +\left(c_{41} d_{4 A A 1}^{a b c d}+c_{43} \delta^{a c} \delta^{b d}\right)\left(\partial J^{c} J^{d}-\partial J^{d} J^{c}-\frac{1}{3} i f^{c d e} \partial^{2} J^{e}\right) \\
& +\left(c_{51} f^{a b c} \delta^{d e}+c_{52} d_{51}^{a b c d e}+c_{53} d_{52}^{a b c d e}\right) \\
& \times \frac{1}{6}\left(J^{c} J^{d} J^{e}+J^{c} J^{e} J^{d}+J^{e} J^{c} J^{d}+J^{d} J^{c} J^{e}+J^{d} J^{e} J^{c}+J^{e} J^{d} J^{c}\right) \\
& \left.+\left(c_{72} d_{4 A A 2}^{a b c d}+c_{73} d_{4 S A}^{c d a b}\right) J^{c} K^{d}+i \frac{4}{a_{1}} f^{a b c} P^{c}\right](w)+\cdots,
\end{aligned}
$$

\footnotetext{
${ }^{8}$ We have checked that all the structure constants are consistent with each other when we consider the $(N, M)=(6,5)$ case and the $(N, M)=(7,6)$ case.
} 
where the structure constants are given by (3.4), (3.6), (3.9), (2.9), (3.11), (3.14), (3.18), (3.31), (3.33), (3.28), (3.26), (3.24), (3.22), and (3.20) and we present them here

$$
\begin{aligned}
& c_{1}=\frac{8\left(k^{2}-1\right) N(2 k+M+N)}{(2 k+M)}, \quad c_{2}=\frac{4\left(k^{2}-1\right) N(2 k+M+N)}{k(2 k+M)}, \\
& a_{1, C H}=\frac{(2 k+M)\left(-k^{2}+k^{2} M^{2}-2 k N-M N+2 k^{2} M N+k M^{2} N-N^{2}+k M N^{2}\right)}{2\left(k^{2}-1\right) M N(2 k+M+N)}, \\
& c=\frac{\left(-k^{2}+k^{2} M^{2}-2 k N-M N+2 k^{2} M N+k M^{2} N-N^{2}+k M N^{2}\right)}{(k+N)(k+M+N)}, \\
& c_{6}=\frac{k(2 k+M+2 N)}{(2 k+M)} \text {, } \\
& c_{31}=-\frac{4\left(4 k^{3}+4 k^{2} M+k M^{2}+8 k^{2} N+6 k M N+M^{2} N+4 k N^{2}+M N^{2}\right)}{M(2 k+M)^{2}}, \\
& c_{32}=\frac{2 k N(2 k+M+N)}{(2 k+M)^{2}}, \quad c_{33}=-\frac{N(2 k+M+N)}{(2 k+M)}, \\
& c_{34}=-\frac{4 N(2 k+M+N)}{k(2 k+M)}, \quad \quad a_{3, C H}=\frac{2(2 k+M)(k+N)(k+M+N)}{\left(k^{2}-1\right) M N(2 k+M+N)} \text {, } \\
& c_{41}=\frac{1}{k M(2 k+M)^{2}(3 k+2 M)}\left(-24 k^{4}-40 k^{3} M-22 k^{2} M^{2}-4 k M^{3}-48 k^{3} N\right. \\
& -64 k^{2} M N+2 k^{4} M N-28 k M^{2} N+3 k^{3} M^{2} N-4 M^{3} N+k^{2} M^{3} N-24 k^{2} N^{2} \\
& \left.-20 k M N^{2}+k^{3} M N^{2}-4 M^{2} N^{2}+k^{2} M^{2} N^{2}\right) \text {, } \\
& c_{43}=-\frac{2 M N(2 k+M+N)}{k(2 k+M)^{2}} \text {, } \\
& c_{51}=-i \frac{4\left(6 k^{3}+7 k^{2} M+2 k M^{2}+12 k^{2} N+10 k M N+2 M^{2} N+6 k N^{2}+2 M N^{2}\right)}{k M(2 k+M)(3 k+2 M)}, \\
& c_{52}=\frac{2 k N(2 k+M+N)}{3(2 k+M)^{2}(3 k+2 M)}, \quad c_{53}=-\frac{2 N(2 k+M+N)}{3(2 k+M)^{2}}, \\
& c_{72}=-\frac{(2 k+M+2 N)}{(2 k+M)}, \quad c_{73}=i \frac{k(2 k+M+2 N)}{(k+M)(2 k+M)} \text {. }
\end{aligned}
$$

In the last line of the second order pole in (3.34), there exists a quasi primary spin-2 operator. In the first two lines of the first order pole there are descendants for the spin-1 and spin-2 operators. In the next five lines, there are quasi primary spin-3 operators. More precisely, the last one is a primary spin- 3 current where the coefficient $a_{1}$ is the overall factor in (2.14). In general, the quasi primary spin-3 operator in the last line is given by $\left(J^{c} K^{d}-\frac{1}{4} i f^{c d e} \partial K^{e}\right)(w)$. However, the derivative term vanishes when we multiply the tensors of $c_{72}$ and $c_{73}$ terms. $^{9}$

Let us emphasize here that although the operator contents appearing in the right hand side of (3.34) except the spin-3 current are the same as the ones in [2], the structure

\footnotetext{
${ }^{9}$ Due to the symmetric or antisymmetric properties of the right hand side of this OPE, we can obtain the quantities by multiplying the antisymmetric $f$ symbols, the symmetric $d$ symbols, or symmetric Kronecker delta symbols. The $c_{31}-c_{34}$ terms are symmetric, the $c_{41}-c_{43}$ terms are antisymmetric, the $c_{51}-c_{53}$ terms are antisymmetric and the $c_{72}-c_{73}$ terms are antisymmetric under the exchange of the indices $a$ and $b$.
} 
constants are completely different from theirs. We can check that the difference between our results and theirs will provide the factor $(k+2 N)$.

When we take the infinity limit of $k$ after substituting $N=\frac{(1-\lambda)}{\lambda} k$ into the various structure constants (3.35) we have determined, we obtain the corresponding values in terms of $\lambda, k$ and $M$. We present them in appendix C. Although we do not compare here the exact values for the structure constants with the ones in [30], we can check the $k$ dependence as well as $M$ dependence. We observe that their (4.40) - (4.42) are consistent with our results with $\lambda=2$ in appendix $\mathrm{C}$ by focusing on the $k$ dependence. Moreover, our coefficients $c_{31}$ and $c_{51}$ do depend on the factor $\frac{1}{M}$ which can be seen from [30] also. ${ }^{10}$

\section{The OPE between the charged higher spin-2 current and the charged higher spin-3 current}

\subsection{The fifth, fourth and third order poles}

First of all, we can calculate the fifth order pole of the OPE $K^{a}(z) P^{b}(w)$ for the fixed $(N, M)=(5,4)$. It turns out that the nonzero contribution appears when the indices $a$ and $b$ are the same. The coefficients contain $a_{6}, a_{12}$ and $a_{13}$ from $P^{b}(w)$ and moreover the common factor appears in the sum of $a_{12}$ and $a_{13}$. Then this contribution becomes zero from the footnote 5 and (2.22).

For the fourth order pole of the OPE $K^{a}(z) P^{b}(w)$ for the fixed $(N, M)=(5,4)$, the contribution appears in the coefficients, $a_{5}, a_{6}, a_{7}, a_{12}$ and $a_{13}$ of $P^{b}(w)$ and the relevant fields are given by $J^{u(1)}$ and $J^{c}$. Again by substituting the values of (2.22), all these terms are vanishing.

Now we move on the third order pole of $K^{a}(z) P^{b}(w)$ where the nonzero results appear explicitly. The relevant coefficients are given by $a_{3}, a_{5}, a_{7}, a_{8}, a_{11}, a_{12}, a_{13}, a_{16}$, and $a_{17}$. For the calculation of $a_{5}$ terms in (2.14), it is better to rewrite them by using the charged spin-2 current in (2.11) because the first two terms of (2.11), which are equal to the factor of $a_{5}$ terms, can be written in terms of the remaining three quantities. That is,

$$
\begin{aligned}
\delta_{\rho \bar{\sigma}} t_{j \bar{i}}^{c}\left(J^{(\rho \bar{i})} J^{(\bar{\sigma} j)}+J^{(\bar{\sigma} j)} J^{(\rho \bar{i})}\right)(w)= & K^{c}(w)+\frac{N}{(M+2 k)} d^{c d e} J^{d} J^{e}(w) \\
& -\frac{2 N}{k} \sqrt{\frac{M+N}{M N}} J^{c} J^{u(1)}(w) .
\end{aligned}
$$

Then the $a_{5}$ term contains $d^{b c d} J^{d}$ multiplied by the above expression to the right. The nontrivial calculation comes from the OPE between $K^{a}(z)$ and $d^{b c d} J^{d} K^{c}(w)$. Due to the fact that there is a relation in (2.12), the contribution of the third order pole in the above OPE can be obtained from the second order pole of the OPE $K^{e}(z) K^{c}(w)$ and the third order pole of the OPE $K^{a}(z) K^{c}(w)$ we have determined in previous section.

\footnotetext{
${ }^{10}$ We regard $d_{4 S S 2}^{a b c d}$ as $\frac{4}{M} \delta^{a d} \delta^{b c}-f^{a c e} f^{e b d}+i f^{a c e} d^{e b d}+i d^{a c e} f^{e b d}+d^{a c e} d^{e b d}$ by using the symmetric property in the indices of $c$ and $d$ in (3.34) from the general definition in appendix A. Similarly, $d_{51}^{a b c d e}$ is given by $i f^{a b f}\left(\frac{6}{M} \delta^{f c} \delta^{d e}+\frac{3}{2}(i f+d)^{f c g} d^{g d e}\right)$ by imposing the symmetric property between the indices $c, d$ and $e$. We also have $d_{52}^{a b c d e}=d_{51}^{a b c d e}+\left(\frac{12}{M} i f^{c b a} \delta^{d e}+\frac{3}{2} i f^{c b f} d^{a f g} d^{g d e}-\frac{3}{2} i f^{c a f} d^{b f g} d^{g d e}\right)$. Finally, we have $d_{4 A A 2}^{a b c d}=i\left(d^{e a c} f^{b d e}+f^{a c e} d^{e b d}\right)$.
} 
It is also nontrivial to calculate the $a_{8}$ term of (2.14). Then we should calculate the second order pole of the OPE between $K^{c}(z)$ and $\delta_{\rho \bar{\sigma}} \delta_{j \bar{i}}\left(J^{(\rho \bar{i})} J^{(\bar{\sigma} j)}+J^{(\bar{\sigma} j)} J^{(\rho \bar{i})}\right)(w)$ and the third order pole of similar OPE with different index we have obtained in previous section.

Because the $a_{12}$ and $a_{13}$ terms of (2.14) cannot be written in terms of other known quantities, it is rather complicated to extract the corresponding third order poles. Let us consider the OPE between the current $K^{a}(z)$ and the composite operator $\delta_{\rho \bar{\sigma}} t_{j \bar{i}}^{b} J^{(\rho \bar{i})} \partial J^{(\bar{\sigma} j)}(w)$ which is not exactly the $a_{13}$ term because there exists $-\frac{1}{2} N \partial^{2} J^{b}(w)$ from the normal ordering in the above composite operator. That is, the commutator $\delta_{\rho \bar{\sigma}} t_{j \bar{i}}^{b}\left[\partial J^{(\bar{\sigma} j)}, J^{(\rho \bar{i})}\right]$ provides the above second derivative term although there are other two terms and the OPEs with $K^{a}(z)$ do not contribute to the final result.

For the coefficient $a_{12}$ term, we have the following relation

$$
\begin{aligned}
\delta_{\rho \bar{\sigma}} t_{j \bar{i}}^{b} \partial J^{(\rho \bar{i})} J^{(\bar{\sigma} j)}(w)= & \frac{1}{2} \partial K^{b}(w)-\delta_{\rho \bar{\sigma}} t_{j \bar{i}}^{b} J^{(\rho \bar{i})} \partial J^{(\bar{\sigma} j)}(w)-\frac{N}{2} \partial^{2} J^{b}(w) \\
& +\frac{N}{2(2 k+M)} d^{b c d} \partial\left(J^{c} J^{d}\right)(w)-\frac{N}{k} \sqrt{\frac{M+N}{M N}} \partial\left(J^{b} J^{u(1)}\right)(w) .
\end{aligned}
$$

For the second term of (4.2), we have analyzed them in the context of $a_{13}$ term in previous paragraph. It is easy to observe that the third order pole from the OPE between $K^{a}(z)$ and $\partial K^{b}(w)$ is given by $\left(\partial\left(K^{a} K^{b}\right)_{\text {pole-3 }}+2\left(K^{a} K^{b}\right)_{\text {pole-2 }}\right)(w)$ from the previous section.

For the $a_{17}$ term of (2.14), in general, there are quintic products in the $f$ and $d$ symbols. After collecting the three products here correctly we are left with $f$ or $d$ symbols and we can further use the identities between the triple products by combining these single $f$ or $d$ symbols with the remaining quadratic products between them.

It turns out that the third order pole, by collecting the above results, is summarized by

$$
\begin{aligned}
K^{a}(z) & \left.P^{b}(w)\right|_{\frac{1}{(z-w)^{3}}}=2 M a_{3} i f^{a b c} K^{c}(w) \\
& +a_{5}\left[-\frac{1}{k M(2 k+M)} 2 N\left(-8 k^{2}+4 k M-4 k^{3} M+4 M^{2}-2 k^{2} M^{2}-8 k N+4 M N\right.\right. \\
& \left.-2 k^{2} M N+k M^{2} N\right) i d^{a c e} f^{e b d} J^{c} J^{d}-\frac{1}{k(2 k+M)} N\left(8 k^{2}-4 k M+4 k^{3} M-4 M^{2}\right. \\
& \left.+2 k^{2} M^{2}+8 k N-4 M N+2 k^{2} M N-k M^{2} N\right) d^{a b c} \partial J^{c} \\
& +\frac{\left(M^{2}-4\right)\left(4 k^{2}+2 k M+4 k N+M N\right)}{M(2 k+M)}\left(N i f^{a b c} \partial J^{c}+\frac{2 N}{k} \sqrt{\frac{M+N}{M N}} i f^{a b c} J^{c} J^{u(1)}\right. \\
& \left.\left.+2 i f^{a b c} \delta_{\rho \bar{\sigma}} t_{j \bar{i}}^{c} J^{(\bar{\rho} \bar{i})} J^{(\bar{\sigma} j)}\right)\right](w)+\frac{4\left(k^{2}-1\right) N(2 k+M+N)}{k(2 k+M)} a_{7} i f^{a b c} J^{c} J^{u(1)}(w) \\
& +a_{8}\left[\frac{4 N}{k} \sqrt{\frac{M+N}{M N}}(2 k+M+2 N) i f^{a b c} J^{u(1)} J^{c}-2 N \frac{(2 k+M+2 N)}{(2 k+M)} i f^{a b c} d^{c d e} J^{d} J^{e}\right. \\
& \left.+4(2 k+M+2 N) i f^{a b c} \delta_{\rho \bar{\sigma}} t_{\bar{j} \bar{i}}^{c} J^{(\rho \bar{i})} J^{(\bar{\sigma} j)}+2 N(2 k+M+2 N) i f^{a b c} \partial J^{c}\right](w)
\end{aligned}
$$




$$
\begin{aligned}
& +\left[-M a_{11}+2 a_{16}+\left(M^{2}+6\right) a_{17}\right] i f^{a b c} K^{c}(w) \\
& +\left(a_{13}-a_{12}\right)\left[-2 \sqrt{\frac{M+N}{M N}}\left(k N+3 N^{2}-50\right) \delta^{a b} \partial J^{u(1)}\right. \\
& +\frac{N\left(6 k^{3}+3 k^{2} M+2 k^{2} N-4 k-2 M-2 N\right)}{k(2 k+M)} i f^{a b c} \partial J^{c}+\frac{k N(2 k+M+2 N)}{(2 k+M)} d^{a b c} \partial J^{c} \\
& +\frac{\left(4 k^{3}+2 k^{2} M-k M N-4 k-2 M-2 N\right)}{k(2 k+M)} i f^{a b c} \delta_{\rho \bar{\sigma}} t_{j \bar{i}}^{c} J^{(\rho \bar{\imath})} J^{(\bar{\sigma} j)} \\
& +\frac{\left(4 k^{2}+2 k M+4 k N+M N\right)}{2 k+M} d^{a b c} \delta_{\rho \bar{\sigma}} t_{j \bar{i}}^{c} J^{(\rho \bar{i})} J^{(\bar{\sigma} j)}+\frac{4(k+N)}{M} \delta^{a b} \delta_{\rho \bar{\sigma}} \delta_{j \bar{i}} J^{(\rho \bar{i})} J^{(\bar{\sigma} j)} \\
& -\frac{2(k+N)(M+N)}{k M} \delta^{a b} J^{u(1)} J^{u(1)}-\frac{N^{2}}{k} \sqrt{\frac{M+N}{M N}} i f^{a b c} J^{u(1)} J^{c} \\
& +\sqrt{\frac{M+N}{M N} \frac{N\left(4 k^{2}+2 k M+4 k N+M N\right)}{k(2 k+M)} d^{a b c} J^{u(1)} J^{c}-\frac{2 N(k+M+N)}{k M} J^{a} J^{b}} \\
& \left.+\frac{N}{2}\left(i f-\frac{2 k+M+2 N}{2 k+M} d\right)(i f+d)^{d b c} J^{e} J^{d}-2 \delta^{a b} J^{\alpha} J^{\alpha}+N i f^{a b c} K^{c}\right](w) \\
& +a_{12}\left[\frac{1}{2} \partial\left(K^{a} K^{b}\right)_{p o l e-3}+\left(K^{a} K^{b}\right)_{p o l e-2}+\frac{M N}{(2 k+M)} d^{a b c} K^{c}\right](w) .
\end{aligned}
$$

We expect that the spin of third order pole is given by 2 and it is natural to consider $K^{c}(w)$ term. Let us focus on the term $i f^{a b c} J^{c} J^{u(1)}(w)$ in (4.3) by remembering the explicit form in (2.11). We obtain the following result

$$
\begin{aligned}
& \frac{4 M N}{k} \sqrt{\frac{M+N}{M N}} a_{3}+\frac{2\left(M^{2}-4\right) N\left(4 k^{2}+2 k M+4 k N+M N\right)}{M k(2 k+M)} \sqrt{\frac{M+N}{M N}} a_{5} \\
& +\frac{4\left(k^{2}-1\right) N(2 k+M+N)}{k(2 k+M)} a_{7}+\frac{4 N(2 k+M+2 N)}{k} \sqrt{\frac{M+N}{M N}} a_{8} \\
& -\frac{2 M N}{k} \sqrt{\frac{(M+N)}{M N}} a_{11}-\frac{N^{2}}{k} \sqrt{\frac{M+N}{M N}} a_{12}+\frac{N^{2}}{k} \sqrt{\frac{M+N}{M N}} a_{13} \\
& +\frac{4 N}{k} \sqrt{\frac{M+N}{M N}} a_{16}+\frac{2\left(M^{2}+6\right) N}{k} \sqrt{\frac{(M+N)}{M N}} a_{17} .
\end{aligned}
$$

Note that the $K^{c}(w)$ term in (4.3) can participate in the expression of (4.4). By substituting the coefficients in (2.22) into the above (4.4), we obtain the final coefficient of $K^{c}(w)$ in the third order pole.

Therefore, finally we determine the third order pole of the OPE $K^{a}(z) P^{b}(w)$ as follows:

$$
\left(K^{a} P^{b}\right)_{\text {pole-3 }}=\frac{\left(k^{2}-4\right)(2 k+M)(k+2 N)(3 k+2 M+2 N)}{2 k(k+M)(3 k+2 M)} a_{1} i f^{a b c} K^{c}(w) .
$$

Because the factor $(k+2 N)$ appears in all the coefficients except $a_{1}$ and $a_{2}$ in the spin-3 current, it is obvious to see that this factor appears in (4.5). 


\subsection{The second order pole}

\subsubsection{Complete second order pole in the coset realization}

For $a_{1}$ term in the spin-3 current, we should calculate the OPEs between the first order poles of the first OPE in (3.1) and $J^{(\bar{\sigma} j)}$. Compared to the OPE $K^{a}(z) W^{(3)}(w)$ associated with $b_{7}$ term, the $a_{1}$ term of (2.14) contains the generator $t_{\rho \bar{\sigma}}^{\alpha}$ rather than $\delta_{\rho \bar{\sigma}}$. In this case, we have similar relations to (3.2) where the indices $a, b, c, \cdots$ are replaced by $\alpha, \beta, \gamma, \cdots$ and $M$ is replaced by $N$. The identities involving $f$ or $d$ symbols for $\mathrm{SU}(N)$ are used.

For $a_{5}$ term, from the previous relation in (4.1), we need to calculate the first and second order poles of the OPE between the charged spin- 2 current. For the former, due to the additional quadratic product of $f$ and $d$ symbols, the identities involving $f f f d, f d f d$, $f f d d$ and $f d d d$ can be used [24, 25].

For $a_{7}$ term, by using the previous relation in (4.1) where the index $c$ is replaced by $b$, we can calculate the OPEs between $K^{a}$ and the right hand sides of (4.1). Then as before, the second order pole of the OPE between $K^{a}(z)$ and $K^{b}(w)$ can be used.

For $a_{8}$ term, as an alternative method, we can use the stress energy tensor and the second and third terms of (2.7) can be written as

$$
\begin{aligned}
& \frac{1}{2(k+N+M)}\left(\delta_{\rho \bar{\sigma}} \delta_{j \bar{i}} J^{(\rho \bar{i})} J^{(\bar{\sigma} j)}+\delta_{\rho \bar{\sigma}} \delta_{j \bar{i}} J^{(\bar{\sigma} j)} J^{(\bar{\rho} \bar{i})}\right)= \\
& T-\frac{1}{2(k+N+M)}\left[J^{\alpha} J^{\alpha}+J^{a} J^{a}+J^{u(1)} J^{u(1)}\right]-\frac{1}{2(k+N)} J^{\alpha} J^{\alpha}-\frac{1}{2 k} J^{u(1)} J^{u(1)} .
\end{aligned}
$$

We can regard the $a_{8}$ term as the product of $J^{b}$ with the right hand side of (4.6). Then the nonzero contributions of the OPE with $K^{a}$ can be calculated from the $T$ term and $J^{c} J^{c}$ terms in (4.6) by using (2.12) and (2.13) because the OPEs between $K^{a}$ and both $J^{\alpha}$ and $J^{u(1)}$ do not have any singular terms.

For $a_{12}$ term, due to the relation in (4.2), the second order pole from the OPE between $K^{a}(z)$ and $\partial K^{b}(w)$ is given by $\frac{1}{2}\left(\partial\left(K^{a} K^{b}\right)_{\text {pole-2 }}+\left(K^{a} K^{b}\right)_{\text {pole- } 1}\right)(w)$ from the previous section.

For $a_{13}$ term, the second order pole of the first OPE in (3.1) can combine with $\partial J^{(\bar{\sigma} j)}$ and similarly the operator $J^{(\rho \bar{i})}$ can be multiplied by the second order pole of the OPE $K^{a}(z)$ and $\partial J^{(\bar{\sigma} j)}(w)$. Moreover, there are also contributions from the second order pole between the first order pole of the first OPE in (3.1) and $\partial J^{(\bar{\sigma} j)}$ and contributions from the second order pole between $K^{a}$ and $\partial J^{(\bar{\sigma} j)}$.

For $a_{17}$ term, the identities for the quartic products in the $f$ and $d$ symbols are used $[24,25]$.

We present the complete second order pole in appendix D.

\subsubsection{How to rearrange the second order pole}

At first sight, because the spin is given by 3 in this particular pole, we do not expect that there should be other independent spin-3 current. It is natural to consider the possibility of spin-3 currents, $P^{c}(w)$ and $W^{(3)}(w)$ with an appropriate additional $\mathrm{SU}(M)$ invariant tensors because the right hand side of the OPE $K^{a}(z) P^{b}(w)$ should contain the free indices 
$a$ and $b$. Of course, the descendant of (4.5) with fixed known coefficient should also appear in the right hand side

$$
\frac{1}{4} \frac{\left(k^{2}-4\right)(2 k+M)(k+2 N)(3 k+2 M+2 N)}{2 k(k+M)(3 k+2 M)} a_{1} i f^{a b c} \partial K^{c} .
$$

The nontrivial things to check explicitly is to write down the remaining composite operators in terms of the known currents for generic $N, M$ and $k$.

The simplest term we can consider is the $b_{3}$ term of $W^{(3)}(w)$ in (2.25). From the $a_{7}$ term in the second order pole in the OPE $K^{a}(z) P^{b}(w)$, the corresponding cubic term in $J^{u(1)}, \delta^{a b} J^{u(1)} J^{u(1)} J^{u(1)}$, is given by $J^{u(1)}\left(K^{a} K^{b}\right)_{\text {pole-2 }}(w)$ and the coefficient is

$$
-\frac{4(k+N)(M+N)}{k M} a_{7} .
$$

By substituting the $a_{7}$ in (2.22) into (4.8), then this leads to $-\frac{b_{1}}{a_{1}} b_{3}$ with (2.32). This implies that there should be

$$
-\delta^{a b} \frac{a_{1}}{b_{1}} W^{(3)}(w)
$$

in the second order pole of the OPE we are considering.

We can check also other simple term. For example, the $a_{2}$ term of (2.14), $d^{a b c} J^{\alpha} J^{\alpha} J^{c}(w)$, can be seen from both $a_{1}$ and $a_{5}$ terms in the second order pole. They are given by

$$
\frac{2(2 k+M+N)}{(2 k+M)} a_{1}-4 a_{5}
$$

By substituting the $a_{5}$ value in (2.22) into (4.10), this can be written as

$$
\frac{k(3 k+2 M)(2 k+M+2 N)}{(k+M)(2 k+M)} a_{2}
$$

where the relation (2.22) is used. Then the second order pole should contain, from (4.11),

$$
\frac{k(3 k+2 M)(2 k+M+2 N)}{(k+M)(2 k+M)} d^{a b c} P^{c}(w) .
$$

After subtracting (4.7), (4.9), and (4.12) from the second order pole, we have checked that we are left with the following seven terms for fixed $(N, M)=(5,4)$

$$
\begin{aligned}
& d_{30} J^{a} K^{b}(w)+d_{32} J^{b} K^{a}(w)+d_{33} f^{\text {ace }} f^{b d e} J^{c} K^{d}(w) \\
& \quad+d_{38} d^{a c e} d^{b d e} J^{d} K^{c}(w)+d_{39} d^{a c e} d^{b d e} J^{c} K^{d}(w)+d_{41} \delta^{a b} J^{c} K^{c}(w)+d_{42} i f^{a b c} \partial K^{c}(w),
\end{aligned}
$$

where the ordering in the coefficients is not important. Of course, these coefficients are known for the above fixed values of $(N, M)$. We have obtained (4.13) by assuming the possible terms with arbitrary coefficients in the right hand side of the second order in the OPE. Note that the above terms (4.13) also arise in the coefficient of $a_{17}$ term of the second order pole. This implies that the second order pole can be written in terms of the known currents we mentioned before. 
Then the next thing we should consider is to determine the above seven undetermined coefficients in terms of $N, M$ and $k$. Let us consider the $d_{33}$ term in (4.13). Recall that there exists a relation we mentioned several times before

$$
f^{a b c} f^{c d e}=-\frac{4}{M}\left(\delta^{a e} \delta^{b d}-\delta^{a d} \delta^{b e}\right)-\left(d^{b d c} d^{c a e}-d^{a d c} d^{c b e}\right) .
$$

When we meet the $f f$ terms, we should always use this identity in order to collect the independent terms. Then by remembering the spin-2 current, the $d_{33}$ term has $d^{\text {abe }} d^{\text {ecd }} J^{c} K^{d}(w)$, where we can see $\frac{2 N}{k} \sqrt{\frac{M+N}{M N}} d^{a b e} d^{e c d} J^{c} J^{d} J^{u(1)}(w)$. We collect the corresponding terms in the second order pole as follows:

$$
-\frac{4 N}{k} \sqrt{\frac{M+N}{M N}} a_{3}-\frac{M N}{(2 k+M)} a_{9}-\frac{2 N}{k} \sqrt{\frac{M+N}{M N}} a_{11}+N a_{7} .
$$

Note that there are also contributions from $a_{17}$ term we do not write down here but they are cancelled each other. It turns out that the $f f$ term with above cubic operators in $J^{u(1)}\left(K^{a} K^{b}\right)_{\text {pole-2 }}$ provides the final contribution with the help of (4.14). This should be equal to

$$
\frac{k(3 k+2 M)(2 k+M+2 N)}{(k+M)(2 k+M)} a_{9}+\frac{2 N}{k} \sqrt{\frac{M+N}{M N}} d_{33},
$$

where the first term comes from (4.12). Therefore, we determine the coefficient $d_{33}$, by using (4.15) and (4.16) together with (2.22), as follows:

$$
d_{33}=\frac{(2 k+M)^{2}(k+2 N)(3 k+2 M+2 N)}{4(k+M)^{2}(3 k+2 M)} a_{1},
$$

which can be substituted into (4.13).

We can move on the term $\delta^{a b} J^{c} K^{c}$ where there exists $\delta^{a b} J^{c} J^{c} J^{u(1)}$ with an appropriate coefficient concerning on the coefficient $d_{41}$. From the second order pole, we have

$$
-\frac{16 N}{k M} \sqrt{\frac{M+N}{M N}} a_{3}+\frac{4 N}{M} a_{7}-\frac{8 N}{k M} \sqrt{\frac{M+N}{M N}} a_{11}-\frac{12 N}{k} \sqrt{\frac{M+N}{M N}} a_{17},
$$

which (there are two contributions from the $a_{17}$ term with (4.14) and the final result by summing over them is given as above) is equal to

$$
-\frac{a_{1}}{b_{1}} b_{5}+\frac{8 N}{k M} \sqrt{\frac{M+N}{M N}} d_{33}+\frac{2 N}{k} \sqrt{\frac{M+N}{M N}} d_{41} .
$$

The first term is obtained from (4.9). By equating these two (4.18) and (4.19) together with (4.17), we have determined the corresponding coefficient as follows:

$$
d_{41}=-\frac{k(2 k+M)(k+2 N)(3 k+2 M+2 N)}{(k+M)^{2}(k+2 M)(3 k+2 M)} a_{1} .
$$

Now this can be substituted into the (4.13) again. 
We consider the $d_{30}$ term where there exists the derivative term $J^{a} \partial J^{b}(w)$. Recall that there is a relation from the footnote 4 . On the one hand, we have the following result

$$
N\left[-6 a_{17}+\frac{4}{M} a_{11}+\frac{8}{M} a_{8}-\frac{4\left(4-M^{2}\right)}{M^{2}} a_{5}-\frac{2(k+M+N)}{k M} a_{13}\right] .
$$

There are two contributions from $a_{17}$ term as before. For the contributions from $a_{8}$ and $a_{11}$, the previous relation (4.14) is used. Note that by combining the contributions in the coefficient $\left(a_{13}-a_{12}\right)$ and the coefficient $a_{12}$, the final contribution from $a_{12}$ term becomes zero. Then we do not have any contributions from $a_{12}$ term in (4.21). On the other hand, this should be equal to

$$
N d_{30} \text {. }
$$

Note that in (4.21), the relation of (4.14) is used in the second, third and fourth terms of (4.21). Then from (4.21) and (4.22), the coefficient can be determined

$$
d_{30}=-\frac{\left(k^{2}+3 k M+M^{2}+4\right)(k+2 N)(3 k+2 M+2 N)}{k M(k+M)(3 k+2 M)} a_{1},
$$

which can be substituted into the (4.13).

Let us look at the $d_{32}$ term where we have the derivative term $J^{b} \partial J^{a}(w)$ with the footnote 4 . We can collect the possible terms as follows:

$$
\begin{gathered}
N\left[\frac{2\left(M^{2}+4\right)}{M} a_{3}-\frac{4\left(M^{2}-4\right)}{M^{2}} a_{5}+\frac{2\left(2 k M+M^{2}+2 M N-4\right)}{M} a_{8}\right. \\
\left.-\frac{2(k+M+N)}{k M} a_{12}-\frac{2(2 k+M+N)}{k M} a_{13}+24 a_{17}\right] .
\end{gathered}
$$

There are two contributions from both $a_{3}$ term and $a_{8}$ term and the final result can be written as above. The contribution from $a_{5}$ term also appears in $i f^{a d e} d^{b c d}\left(K^{e} K^{c}\right)_{\text {pole- } 1}(w)$. Note that the additional contribution from $a_{12}$ term can be found in $\frac{1}{2}\left(K^{a} K^{b}\right)_{\text {pole- } 1}(w)$. From the three places of $a_{17}$ term, the final result for this coefficient is given above. On the other hand, there exists

$$
N\left(-d_{32}+\frac{4}{M} d_{33}\right)
$$

Then we arrive at the following result, by using (4.24) and (4.25) which are equal to each other,

$$
d_{32}=\frac{\left(k^{3}-2 k^{2} M-3 k M^{2}+4 k-M^{3}+4 M\right)(k+2 N)(3 k+2 M+2 N)}{k M(k+M)^{2}(3 k+2 M)} a_{1} .
$$

Then this coefficient can be substituted in (4.13).

For the $d_{39}$ term, we have the derivative term $d^{a c e} d^{e b d} J^{c} \partial J^{d}(w)$. We can collect the possible terms as follows:

$$
N\left[-\frac{\left(8 k+M^{2} N+4 M\right)}{M(2 k+M)} a_{5}+2 a_{8}-\frac{(2 k+M+2 N)}{2(2 k+M)} a_{13}+a_{11}\right] .
$$


The two contributions from $a_{12}$ are cancelled each other. Similarly, those from $a_{17}$ can be also cancelled. After simplifying the contributions from the $a_{5}$ term, the net result comes from $i f^{a d e} d^{b c d}\left(K^{e} K^{c}\right)_{\text {pole-1 }}$ as above. This should be equal to

$$
N d_{39}
$$

Then from (4.27) and (4.28) by taking them to be equal to each other, we obtain

$$
d_{39}=-\frac{\left(k^{2}+k M+4\right)(k+2 N)(3 k+2 M+2 N)}{4 k(k+M)(3 k+2 M)} a_{1},
$$

which can be substituted into the (4.13).

Similarly, the corresponding terms for the $d_{42}$ term which involves various different kind of coefficients by considering the term $f^{a b c} J^{c} \partial J^{u(1)}(w)$ can be obtained

$$
\begin{aligned}
N \sqrt{\frac{M+N}{M N}}[ & \frac{4 M}{k} a_{3}+\frac{2\left(M^{2}-4\right)(k+N)}{k M} a_{5}+\frac{4(k+N)}{k} a_{8} \\
& \left.\quad-\frac{2 M}{k} a_{11}-\frac{(k+N)}{k} a_{12}+\frac{N}{k} a_{13}+\frac{4}{k} a_{16}+\frac{2\left(M^{2}+6\right)}{k} a_{17}\right] .
\end{aligned}
$$

The $a_{3}$ term and $a_{11}$ term can be obtained by changing the ordering of the two operators. After we simplify all the contributions from the $a_{5}$ term, the final result comes from $i f^{a d e} d^{b c d}\left(K^{e} K^{c}\right)_{\text {pole-1 }}$ as above. Note that there are contributions from various places corresponding to the $a_{12}$ and $a_{13}$ terms. Then the above should be equal to

$$
\frac{2 N}{k} \sqrt{\frac{M+N}{M N}}\left[\frac{1}{4} \frac{\left(k^{2}-4\right)(2 k+M)(k+2 N)(3 k+2 M+2 N)}{2 k(k+M)(3 k+2 M)} a_{1}+d_{42}\right] .
$$

Then it is easy to obtain the following result from (4.30) and (4.31)

$$
d_{42}=-\frac{(k-2)(k+2) M(k+2 N)(3 k+2 M+2 N)}{8 k(k+M)(3 k+2 M)} a_{1} .
$$

This can be substituted in (4.13).

For the final coefficient, we use a little different method. It is straightforward to calculate the OPEs between $T(z)$ and each term of (4.13) respectively. The third order pole of these (except the $d_{41}$ term) has the form $i f^{a b c} K^{c}(w)$. By requiring that the expression (4.13) should be a quasi primary operator, there exists for the vanishing of the third order pole

$$
d_{30}-d_{32}+M d_{33}-\frac{\left(M^{2}-4\right)}{M} d_{38}+\frac{\left(M^{2}-4\right)}{M} d_{39}+4 d_{42}=0
$$

This (4.33) implies that we obtain the coefficient $d_{38}$ by using (4.23), (4.26), (4.17), (4.29), and (4.32) as follows:

$$
d_{38}=-\frac{\left(k^{2}+k M+4\right)(k+2 N)(3 k+2 M+2 N)}{4 k(k+M)(3 k+2 M)} a_{1},
$$


which can be substituted in (4.13). We can also check the above result by following previous method after extracting the corresponding terms from the second order pole.

Therefore, by substituting (4.23), (4.26), (4.17), (4.34), (4.29), (4.20), and (4.32) into the previous expression (4.13) the known quasi spin-3 operator can be written as

$$
\begin{aligned}
Q^{a b}(w) \equiv & \frac{N(k+2 N)(3 k+2 M+2 N)}{6(k+M)(3 k+2 M)} a_{1}\left[-\frac{6\left(4+k^{2}+3 k M+M^{2}\right)}{k M N} J^{a} K^{b}\right. \\
& +\frac{6\left(4 k+k^{3}+4 M-2 k^{2} M-3 k M^{2}-M^{3}\right)}{k(k+M) M N} J^{b} K^{a} \\
& +\frac{3(2 k+M)^{2}}{2(k+M) N} f^{a c e} f^{b d e} J^{c} K^{d}+\frac{3\left(4 k+k^{3}+4 M\right)}{2 k(k+M) N} d^{a c e} d^{b d e} J^{d} K^{c} \\
& -\frac{3\left(4+k^{2}+k M\right)}{2 k N} d^{a c e} d^{b d e} J^{c} K^{d} \\
& \left.-\frac{6 k(2 k+M)}{(k+M)(k+2 M) N} \delta^{a b} J^{c} K^{c}-\frac{3\left(k^{2}-4\right) M}{4 k N} i f^{a b c} \partial K^{c}\right](w) .
\end{aligned}
$$

By multiplying $f$ or $d$ symbols into (4.35), we obtain the primary operator having a single index. ${ }^{11}$ We observe that the OPEs between the operator $(4.35)$ and $J^{u(1)}(w)$ (or $\left.J^{\alpha}(w)\right)$ are regular because this operator consists of the spin- 1,2 currents. We can check the primary condition for the spin-3 operator having the two indices

$$
T(z) Q^{a b}(w)=\frac{1}{(z-w)^{2}} 3 Q^{a b}(w)+\frac{1}{(z-w)} \partial Q^{a b}(w)+\cdots .
$$

Therefore, the second order pole can be described as

$$
\begin{aligned}
\left(K^{a} P^{b}\right)_{\text {pole-2 }}(w)= & \frac{1}{4} \frac{\left(k^{2}-4\right)(2 k+M)(k+2 N)(3 k+2 M+2 N)}{2 k(k+M)(3 k+2 M)} a_{1} i f^{a b c} \partial K^{c}(w) \\
& -\delta^{a b} \frac{a_{1}}{b_{1}} W^{(3)}(w)+\frac{k(3 k+2 M)(2 k+M+2 N)}{(k+M)(2 k+M)} d^{a b c} P^{c}(w)+Q^{a b}(w),
\end{aligned}
$$

where the spin-3 primary (4.36) operator $Q^{a b}(w)$ is given by (4.35). Compared to the third order pole in (4.3) where there exists the term $f^{a b c} K^{c}(w)$, the second order pole in (4.37) has both $d^{a b c} P^{c}(w)$ and $\delta^{a b} W(w)$ terms which are symmetric under the interchange between the index $a$ and $b$ as well as some descendant. We expect that this alternating feature will appear through the whole singular terms in the given OPEs.

\subsection{The first order pole and charged quasi primary spin-4 current}

Compared to other singular terms described in previous subsections, the first order pole can be obtained by simple contraction between the operators. We present this in appendix E. We expect that there exists a new quasi spin- 4 current in this singular term. In the third

\footnotetext{
${ }^{11}$ Under the large $k$ limit, the coefficients in (4.35) become $\frac{k\left(\lambda^{2}-4\right)}{3 \lambda^{2} M} a_{1},-\frac{k\left(\lambda^{2}-4\right)}{3 \lambda^{2} M} a_{1},-\frac{k\left(\lambda^{2}-4\right)}{3 \lambda^{2}} a_{1}$, $-\frac{k\left(\lambda^{2}-4\right)}{12 \lambda^{2}} a_{1}, \frac{k\left(\lambda^{2}-4\right)}{12 \lambda^{2}} a_{1}, \frac{2\left(\lambda^{2}-4\right)}{3 \lambda^{2}} a_{1}$, and $\frac{k\left(\lambda^{2}-4\right) M}{24 \lambda^{2}} a_{1}$ respectively.
} 
order pole, the field content is given by $i f^{a b c} K^{c}(w)$. Along the line of this behavior, by introducing the following quantity

$$
i f^{a b c}\left(K^{a} P^{b}\right)_{\text {pole- }-1}(w) \equiv R^{c}(w),
$$

and subtracting the corresponding quantity from the descendants with a multiplication of $i f^{a b c}$, a new quasi spin- 4 current is given by ${ }^{12}$

$$
\begin{aligned}
\hat{R}^{c}(w) \equiv & R^{c}(w)-\frac{1}{3} i f^{a b c} \partial Q^{a b}(w) \\
& +\frac{M\left(k^{2}-4\right)(2 k+M)(k+2 N)(3 k+2 M+2 N)}{20 k(k+M)(3 k+2 M)} a_{1} \partial^{2} K^{c}(w),
\end{aligned}
$$

where $Q^{a b}(w)$ is given by (4.35). When the $f$ symbols meet the Kronecker delta or $d$ symbols by two index contractions, we get zero. Let us emphasize that this new quasi spin-4 current is completely determined via the left hand side of (4.38) from appendix E and the two terms of the right hand side of (4.39).

Then the first order pole is given by

$$
\begin{aligned}
\left(K^{a} P^{b}\right)_{\text {pole- } 1}(w)= & \frac{1}{20} \frac{\left(k^{2}-4\right)(2 k+M)(k+2 N)(3 k+2 M+2 N)}{2 k(k+M)(3 k+2 M)} a_{1} i f^{a b c} \partial^{2} K^{c}(w) \\
& -\frac{1}{3} \delta^{a b} \frac{a_{1}}{b_{1}} \partial W^{(3)}(w)+\frac{1}{3} \frac{k(3 k+2 M)(2 k+M+2 N)}{(k+M)(2 k+M)} d^{a b c} \partial P^{c}(w) \\
& +\frac{1}{3} \partial Q^{a b}(w)+R^{a b}(w),
\end{aligned}
$$

where we introduce the operator $R^{a b}(w)$ which is given by the first order pole subtracted by the descendant terms. Then how we can connect this with the above quasi spin- 4 current? From the relation

$$
\left(K^{a} P^{b}\right)_{\text {pole- } 1}(z)=-\frac{1}{2 M} i f^{a b c} R^{c}(w)+S^{a b}(w),
$$

and by equating (4.40) and (4.41) each other, we can write down the above $R^{a b}(w)$ in terms of $R^{c}(w), S^{a b}(w)$ and other known operators. Note that the above behavior can be seen from the first order pole of the OPE between the spin-2 current and itself (3.34). This (4.41) can be seen from the fixed $(N, M)=(5,4)$ case. In other words,

$$
R^{a b}=-\frac{1}{2 M} i f^{a b c} \hat{R}^{c}(w)+\text { other terms. }
$$

Therefore, the $R^{a b}(w)$ contains the previous quasi spin-4 current in (4.39) and can be treated as similar quasi spin- 4 current with two indices. We can easily see that the overall factor $\frac{1}{2 M}$ in (4.42) can be checked by multiplying $i f^{a b d}$ into (4.41) and using (4.38)

\footnotetext{
${ }^{12}$ We have the nontrivial fourth order pole in the OPE between $T(z)$ and $\hat{R}^{a}(w)$ which is given by $-\frac{288(k-2)(k+2)^{2}(k+6)(k+10)}{5 k(k+4)(3 k+8)} a_{1} K^{a}(w)$ for $(N, M)=(5,4)$. By adding $i f^{a b c}\left(J^{b} \partial K^{c}-2 \partial J^{b} K^{c}-\right.$ $\left.\frac{i}{10} f^{b c d} \partial^{2} K^{d}\right)(w)$ into this new quasi primary spin-4 current and removing the fourth order pole above, we can make a primary spin-4 current at least for $(N, M)=(5,4)$. For generic $(N, M)$ case, we should find out the above fourth order pole for the general case. Then we can easily fix the above relative coefficient we want to add above. See also the footnote 13.
} 
and (4.39). When we substitute (4.42) into (4.40) and use (4.39), then the above "other terms" can be read off explicitly. Note that the left hand side of (4.40) is given by appendix $\mathrm{E}$ in terms of coset realization.

Although the expression of $S^{a b}(w)$ can be determined for general $N$ and $M$ by following the procedure we have described in the construction of $Q^{a b}(w)$ in (4.35), it will be rather nontrivial and complicated due to the fact that there are tensorial structures having five indices. Instead we present them for fixed $(N, M)=(5,4)$ as follows:

$$
\begin{aligned}
&\left.S^{a b}(w)\right|_{N=5, M=4} \equiv-\frac{1}{3} \frac{a_{1}}{b_{1}} \delta^{a b} \partial W^{(3)}(w)+\frac{k(k+7)}{(k+4)} d^{a b c} \partial P^{c}(w) \\
&+\frac{(k+7)}{2(k+2)(k+4)}\left[-(3 k+8) d_{4 A A 2}^{d c b a}+i k\left(d_{4 S A}^{c a d b}+d_{4 S A}^{c b d a}\right)\right] J^{c} P^{d}(w) \\
&+\frac{(k+6)(k+10)}{(k+4)^{2}(3 k+8)} a_{1}\left[-\frac{3(7 k-4)}{40 k} d_{51}^{d b e c a}-\frac{3(7 k-4)}{40 k} d_{51}^{e a d c b}\right. \\
&+\frac{3\left(5 k^{2}+33 k+4\right)}{80 k} d_{51}^{e d c b a}-\frac{3\left(20 k^{2}+59 k+12\right)}{40 k} d_{52}^{d b e c a} \\
&-\frac{3\left(10 k^{2}+21 k-12\right)}{40 k} d_{52}^{e b d c a}-\frac{3\left(15 k^{2}+23 k-36\right)}{80 k} d_{52}^{e d c b a} \\
&\left.+\frac{3 i(k+2)}{4} f^{a b c} \delta^{d e}+\frac{3 i(k+4)}{8} f^{a b e} \delta^{d c}+\frac{3 i(k+4)}{k} f^{a e d} \delta^{b c}\right] J^{c} J^{d} K^{e}(w) \\
&+ \frac{(k+6)(k+10)}{(k+4)^{2}(3 k+8)} a_{1}\left[-\frac{3\left(10 k^{3}+75 k^{2}+346 k+568\right)}{40 k} \partial J^{a} K^{b}\right. \\
&- \frac{3(k+4)^{2}}{8} J^{a} \partial K^{b}+\frac{3\left(10 k^{3}-20 k^{2}-149 k-132\right)}{40 k} \partial J^{b} K^{a}+\frac{3\left(k^{2}-4 k-16\right)}{8} J^{b} \partial K^{a} \\
&+ \frac{3\left(20 k^{3}+165 k^{2}+277 k-44\right)}{40 k} f^{a c e} f^{b d e} \partial J^{c} K^{d}+\frac{3 k(k+2)}{4} f^{a c e} f^{b d e} J^{c} \partial K^{d} \\
&+ \frac{3\left(10 k^{3}+5 k^{2}-78 k-104\right)}{40 k} d^{a c e} d^{e b d} \partial J^{d} K^{c}+\frac{3 k^{2}}{8} d^{a c e} d^{e b d} J^{d} \partial K^{c} \\
&- \frac{3\left(2 k^{3}+6 k^{2}-9 k-20\right)}{8 k} d^{a c e} d^{e b d} \partial J^{c} K^{d}-\frac{3 k(k+4)}{8} d^{a c e} d^{e b d} J^{c} \partial K^{d} \\
&-\left.\frac{3\left(45 k^{3}+233 k^{2}+268 k+32\right)}{40 k(k+8)} \delta^{a b} \partial J^{c} K^{c}-\frac{3 k^{2}}{2(k+8)} \delta^{a b} J^{c} \partial K^{c}\right](w) .
\end{aligned}
$$

It is rather nontrivial to extract this expression without any unwanted terms like as $J^{u(1)}(w), J^{\alpha}(w), J^{(\rho \bar{i})}(w)$, or $J^{(\bar{\sigma} j)}(w)$ explicitly. The other unwanted spin-1 currents can be absorbed in the new quasi primary current in (4.39). Note that the operator contents in (4.43) consist of the spin-1,2,3 currents and some of the derivative terms can be seen from the derivative of $Q^{a b}(w)$ in (4.35). This implies that the right hand side of (4.43) depends on the adjoint operators (or singlet operator) living in $\mathrm{SU}(M) .{ }^{13}$

\footnotetext{
${ }^{13}$ We can check the following properties. The $J^{a} W^{(3)}(w)$ is a primary spin-4 operator and the $J^{a} P^{b}(w)-$ $\frac{1}{6} i f^{a b c} \partial P^{c}(w)$ is a primary operator. On the other hand, $J^{a} \partial K^{b}(w)-2 \partial J^{a} K^{b}(w)-\frac{i}{10} f^{a b c} \partial^{2} K^{c}(w)$ is quasi primary spin- 4 operator and the fourth order pole in the OPE between the stress energy tensor and this operator is given by $-\frac{21}{5} i f^{a b c} K^{c}(w)$.
} 
Moreover, the OPE between the spin- 1 current and the above quasi primary spin- 4 current for fixed $(N, M)=(5,4)$ is described by

$$
\begin{aligned}
J^{a}(z) \hat{R}^{b}(w)= & -\frac{1}{(z-w)^{3}}\left[\frac{28(k-2)(k+2)^{2}(k+6)(k+10)}{5 k(k+4)(3 k+8)} a_{1}\right] i f^{a b c} K^{c}(w) \\
& +\frac{1}{(z-w)^{2}}\left[\frac { ( k + 2 ) ( k + 6 ) ( k + 1 0 ) } { ( k + 4 ) ^ { 2 } ( 3 k + 8 ) } \left(-\frac{(k+4)(7 k+4)}{k} a_{1} J^{a} K^{b}\right.\right. \\
& -\frac{\left(11 k^{2}+16 k-16\right)}{k} a_{1} J^{b} K^{a}+\frac{6(k-4)(k+2)}{k} a_{1} f^{a c e} f^{b d e} J^{c} K^{d} \\
& -\frac{\left(5 k^{2}-8 k-16\right)}{k} a_{1} d^{a c e} d^{e b d} J^{d} K^{c} \\
& -\frac{(k+4)^{2}}{k} a_{1} d^{a c e} d^{e b d} J^{c} K^{d}-\frac{6 k^{2}}{(k+8)} a_{1} \delta^{a b} J^{c} K^{c} \\
& \left.+\frac{(k-2)(k+4)(7 k+24)}{5 k} a_{1} i f^{a b c} \partial K^{c}\right) \\
& \left.-\frac{4 k(k+7)(3 k+8)}{(k+2)(k+4)} d^{a b c} P^{c}\right](w)+\frac{1}{(z-w)} i f^{a b c} \hat{R}^{c}(w)+\cdots
\end{aligned}
$$

We observe that there exists a factor $(k+10)$ which comes from the factor $(k+2 N)$. The first order pole is what we have expected. Contrary to the spin-2,3 currents, there are more singular terms in addition to the first order pole in (4.44). In principle, the above calculation can be done for any $N$ and $M$ but it will take time to complete this computation.

\subsection{The final OPE}

In summary, we present the OPE between the charged spin- 2 current and the charged spin-3 current as follows:

$$
\begin{aligned}
K^{a}(z) P^{b}(w)= & \frac{1}{(z-w)^{3}}\left[\frac{\left(k^{2}-4\right)(2 k+M)(k+2 N)(3 k+2 M+2 N)}{2 k(k+M)(3 k+2 M)}\right] a_{1} i f^{a b c} K^{c}(w) \\
& +\frac{1}{(z-w)^{2}}\left[\frac{1}{4} \frac{\left(k^{2}-4\right)(2 k+M)(k+2 N)(3 k+2 M+2 N)}{2 k(k+M)(3 k+2 M)} a_{1} i f^{a b c} \partial K^{c}\right. \\
& \left.+Q^{a b}-\delta^{a b} \frac{a_{1}}{b_{1}} W^{(3)}+\frac{k(3 k+2 M)(2 k+M+2 N)}{(k+M)(2 k+M)} d^{a b c} P^{c}\right](w) \\
& +\frac{1}{(z-w)}\left[\frac{1}{20} \frac{\left(k^{2}-4\right)(2 k+M)(k+2 N)(3 k+2 M+2 N)}{2 k(k+M)(3 k+2 M)} a_{1} i f^{a b c} \partial^{2} K^{c}\right. \\
& +\frac{1}{3} \partial Q^{a b}-\frac{1}{3} \delta^{a b} \frac{a_{1}}{b_{1}} \partial W^{(3)}+\frac{1}{3} \frac{k(3 k+2 M)(2 k+M+2 N)}{(k+M)(2 k+M)} d^{a b c} \partial P^{c} \\
& \left.+R^{a b}\right](w)+\cdots .
\end{aligned}
$$


In the right hand side of (4.45), there exists an overall factor $a_{1} \cdot{ }^{14}$ Although the explicit form for the quasi primary spin- 4 current $\hat{R}^{c}(w)$ is determined via the known currents and coset operators, the explicit form for the $R^{a b}$ for general $(N, M)$ is not known but it is known for $(N, M)=(5,4)$ because we do not know how appendix (E.1) can be written in terms of the known currents. Their operator contents are known but the relative coefficients are known for $(N, M)=(5,4)$. Or if we interpret $R^{a b}$ itself as the whole new charged quasi primary spin- 4 current (without splitting the quasi primary spin- 4 current with a single free index and others), we do not worry about the fact that this is written in terms of coset realization. ${ }^{15}$

\section{The OPE between the charged spin-3 current and itself with $(N, M)=(5,4)$}

\subsection{The sixth, fifth, fourth order poles}

For the sixth order pole we expect to have the Kronecker delta $\delta^{a b}$ term. For the fifth order pole, there exists $i f^{a b c} J^{c}(w)$ term. For the fourth order pole, there are symmetric $\delta^{a b}$ and $d^{a b c}$ tensor terms and other symmetric tensor terms in (3.34) as well as the descendant term.

\subsection{The third order pole}

We can take the operator contents in the first order pole of (3.34) at the third order pole of this OPE. In other words, in addition to the descendant terms, there are $i f^{a b c} P^{c}(w)$ and the quasi primary spin-3 operator including the stress energy tensor. Compared to (3.34), the other two kinds of quasi primary spin-3 operators do not appear in this OPE.

\subsection{The second and first order poles}

Then we obtain the OPE as follows:

$$
\begin{aligned}
& P^{a}(z) P^{b}(w)=\frac{1}{(z-w)^{6}}\left[\frac{15\left(k^{2}-4\right)\left(k^{2}-1\right)(k+6)(k+10)(2 k+9)}{2 k(k+4)(3 k+8)}\right] a_{1}^{2} \delta^{a b} \\
& +\frac{1}{(z-w)^{5}}\left[\frac{15\left(k^{2}-4\right)\left(k^{2}-1\right)(k+6)(k+10)(2 k+9)}{2 k^{2}(k+4)(3 k+8)}\right] a_{1}^{2} i f^{a b c} J^{c}(w) \\
& +\frac{1}{(z-w)^{4}}\left[\frac{3\left(k^{2}-4\right)(k+5)(k+6)(k+9)(k+10)}{4 k(k+4)} a_{1}^{2} \delta^{a b} T\right. \\
& +\frac{3\left(k^{2}-4\right)(k+6)(k+7)(k+10)}{4(k+4)^{2}} a_{1}^{2} d^{a b c} K^{c} \\
& +\frac{1}{2} \frac{15\left(k^{2}-4\right)\left(k^{2}-1\right)(k+6)(k+10)(2 k+9)}{2 k^{2}(k+4)(3 k+8)} a_{1}^{2} i f^{a b c} \partial J^{c}
\end{aligned}
$$

\footnotetext{
${ }^{14}$ The structure constant in the third order becomes $-\frac{k^{2}\left(\lambda^{2}-4\right)}{3 \lambda^{2}} a_{1}$ when we take the infinity limit of $k$. The one appearing in charged spin- 3 current of the second order pole becomes $\frac{3 k}{\lambda}$.

${ }^{15}$ For the first order pole of the OPE in (3.34), we can treat the sum of four quasi primary spin-3 operators (after subtracting the descendant terms) and a single primary spin-3 current as the whole single quasi primary spin-3 current having two free indices. Then we do not need to specify the above four quasi primary spin-3 currents in terms of multiple products between the known currents.
} 


$$
\begin{aligned}
& +\frac{(k+6)(k+10)}{(k+4)(3 k+8)}\left(\frac{15(k-2)(2 k+9)}{8} a_{1}^{2} d_{4 S S 1}^{a b c d}-\frac{15\left(k^{2}-4\right)(2 k+9)}{8 k} a_{1}^{2} d_{4 S S 2}^{a b c d}\right. \\
& \left.-\frac{3(k-2)\left(180+191 k+44 k^{2}+3 k^{3}\right)}{8 k} a_{1}^{2} \delta^{a b} \delta^{c d}-\frac{15\left(k^{2}-4\right)(2 k+9)}{2 k^{2}} a_{1}^{2} \delta^{a c} \delta^{b d}\right) \\
& \left.\times \frac{1}{2}\left(J^{c} J^{d}+J^{d} J^{c}\right)\right](w) \\
& +\frac{1}{(z-w)^{3}}\left[\frac{1}{2} \frac{3\left(k^{2}-4\right)(k+5)(k+6)(k+9)(k+10)}{4 k(k+4)} a_{1}^{2} \delta^{a b} \partial T\right. \\
& +\frac{1}{2} \frac{3\left(k^{2}-4\right)(k+6)(k+7)(k+10)}{4(k+4)^{2}} a_{1}^{2} d^{a b c} \partial K^{c} \\
& +\frac{1}{6} \frac{15\left(k^{2}-4\right)\left(k^{2}-1\right)(k+6)(k+10)(2 k+9)}{2 k^{2}(k+4)(3 k+8)} a_{1}^{2} i f^{a b c} \partial^{2} J^{c} \\
& +\frac{1}{2} \frac{(k+6)(k+10)}{(k+4)(3 k+8)}\left(\frac{15(k-2)(2 k+9)}{8} a_{1}^{2} d_{4 S S 1}^{a b c d}-\frac{15\left(k^{2}-4\right)(2 k+9)}{8 k} a_{1}^{2} d_{4 S S 2}^{a b c d}\right. \\
& \left.-\frac{3(k-2)\left(180+191 k+44 k^{2}+3 k^{3}\right)}{8 k} a_{1}^{2} \delta^{a b} \delta^{c d}-\frac{15\left(k^{2}-4\right)(2 k+9)}{2 k^{2}} a_{1}^{2} \delta^{a c} \delta^{b d}\right) \\
& \times \frac{1}{2} \partial\left(J^{c} J^{d}+J^{d} J^{c}\right) \\
& +\frac{\left(15 k^{6}+278 k^{5}+1648 k^{4}+2208 k^{3}-10480 k^{2}-37088 k-34560\right)}{2 k(k+4)^{2}(3 k+8)} a_{1} i f^{a b c} P^{c} \\
& \left.+\frac{3\left(k^{2}-4\right)(k+5)(k+6)(k+9)(k+10)}{4 k^{2}(k+4)} a_{1}^{2}\left(T J^{a}-\frac{1}{2} \partial^{2} J^{a}\right)\right](w) \\
& +\mathcal{O}\left(\frac{1}{(z-w)^{2}}\right)+\cdots
\end{aligned}
$$

In the second order pole, there exist spin- 4 quasi primary operators in addition to the descendant terms as usual. We expect that there will be symmetric terms, $\delta^{a b} W^{(4)}(w)$ where the neutral primary spin- 4 current will be presented in next section and $d^{a b c} \hat{R}^{c}(w)$ by recalling the second order pole of (4.45). We observe that in the second order pole there exists a term $J^{\alpha=1} J^{\alpha=1} J^{\alpha=22} J^{\alpha=23}(w)$ which is one of the terms in the neutral primary spin-4 current $W^{(4)}(w)$ for the equal indices $a=b$. So far we do not obtain the explicit form for the second order pole due to the fact that there are two many candidates with various tensorial structures at this singular terms. We expect that there is a new primary field of spin-5 in the first order pole.

\section{The OPE between the uncharged higher spin-3 current and itself}

\subsection{For fixed $(N, M)=(5,4)$ case}

\subsubsection{The sixth, fifth, fourth and third order poles}

We expect that the highest order pole contains the central term. We observe that this contains the factor $(k+10)$ which is given by $(k+2 N)$ for general $N$. There will be no fifth order 
pole because we are considering the OPE between the spin-3 current and itself. The fourth order pole should contain the spin-2 current as usual. It turns out that there is also the quadratic term $J^{a} J^{a}(w)$. Then the third order pole should contain the descendant terms.

\subsubsection{The second and first order poles with the presence of uncharged primary spin-4 current}

The second order pole can provide us to have a new primary current of spin-4. It turns out that the final OPE for fixed $(N, M)=(5,4)$ is given by

$$
\begin{aligned}
W^{(3)}(z) W^{(3)}(w)= & \frac{1}{(z-w)^{6}} \frac{\hat{c}}{3}+\frac{1}{(z-w)^{4}} 2 \hat{T}(w)+\frac{1}{(z-w)^{3}} \partial \hat{T}(w) \\
& +\frac{1}{(z-w)^{2}}\left[\frac{3}{10} \partial^{2} \hat{T}+\frac{16(k+4)(k+5)(k+9)}{3\left(37 k^{3}+216 k^{2}+337 k+510\right)}\left(\hat{T} \hat{T}-\frac{3}{10} \partial^{2} \hat{T}\right)\right. \\
& \left.+W^{(4)}\right](w) \\
& +\frac{1}{(z-w)}\left[\frac{1}{15} \partial^{3} \hat{T}+\frac{1}{2} \frac{16(k+4)(k+5)(k+9)}{3\left(37 k^{3}+216 k^{2}+337 k+510\right)} \partial\left(\hat{T} \hat{T}-\frac{3}{10} \partial^{2} \hat{T}\right)\right. \\
& \left.+\frac{1}{2} \partial W^{(4)}\right](w)+\cdots,
\end{aligned}
$$

where the $b_{1}^{2}$ of the overall constant in the neutral primary spin- 3 current $W^{(3)}$ is fixed as follows:

$$
b_{1}^{2}=\frac{8 k(k+8)}{27\left(k^{2}-4\right)(k+5)^{2}(k+6)(k+9)^{2}(k+10)} .
$$

In (6.2), the requirement we impose is that the central term of (6.1) should be equal to $\frac{\hat{c}}{3}$ with (6.3). As described before, there exists a $J^{a} J^{a}(w)$ term in the fourth order pole. Note that the modified central charge and stress energy tensor are given by

$$
\begin{aligned}
& \hat{c} \equiv c-\frac{15 k}{(k+4)}=\frac{20\left(k^{2}-1\right)(2 k+9)}{(k+4)(k+5)(k+9)}, \\
& \hat{T} \equiv T-\frac{1}{2(k+4)} J^{a} J^{a} .
\end{aligned}
$$

We can easily see that the OPE between the spin- 1 current and the modified stress energy tensor is regular

$$
J^{a}(z) \hat{T}(w)=0+\cdots .
$$

We can calculate the OPE between the stress energy tensor and the $J^{a} J^{a}(w)$ term and this leads to the central charge $\frac{15 k}{(k+4)}$. Due to the fact that there is a relation in (6.4), we obtain the modified central charge is given by (6.3). Therefore, the spin- 1 current is decoupled from the modified stress energy tensor according to (6.4). See also [31] where the $\mathrm{U}(1)$ spin- 1 current is decoupled from the stress energy tensor, spin-3, 4 currents in the specific model. 
We have explicit form for the primary spin- 4 current $^{16}$ as follows:

$$
\begin{aligned}
W^{(4)}(w)= & -\frac{9(k+2)(k+5)(k+9)\left(53 k^{4}+800 k^{3}+3409 k^{2}+3078 k-3600\right)}{2 k(k+8)\left(37 k^{3}+216 k^{2}+337 k+510\right)} b_{1}^{2} \\
& \times J^{\alpha=1} J^{\alpha=1} J^{\alpha=1} J^{\alpha=1}(w)+\text { other } 9330 \text { terms },
\end{aligned}
$$

together with (6.2). We can also check that the regularity between the spin-1 current and this spin-4 current (6.5)

$$
J^{a}(z) W^{(4)}(w)=0+\cdots
$$

This implies that the spin- 1 current is decoupled. We will observe in next section that the regularity between the spin- 1 current and this spin-3 current

$$
J^{a}(z) W^{(3)}(w)=0+\cdots
$$

This fact can be seen from the closure of the OPE between $K^{a}(z)$ and $W^{(3)}(w)$.

Therefore, we have the spin-2,3,4 currents $\hat{T}, W^{(3)}$ and $W^{(4)}$, having the regularity behavior in (6.4), (6.6) and (6.7). The OPE between the spin-2 current and itself takes the standard form with modified central charge. The (quasi)primary condition under the stress energy tensor is preserved when we modify the stress energy tensor because the $J^{a} J^{a}(z)$ term does not spoil the spin-3, 4 currents according to the regularity.

\subsection{For general $(N, M)$ case}

The coefficient appearing in the quasi spin-4 operator $\left(\hat{T} \hat{T}-\frac{3}{10} \partial^{2} \hat{T}\right)$ is fractional function of $k$ and both numerator and denominator are polynomials of $k$. The highest power is given by three. We can express this coefficient in terms of the central charge. It turns out that this is equal to the well known quantity $\frac{32}{(5 \hat{c}+22)}$ for fixed $N$ and $M$ with (6.3). Because the structure constant depends only on the central charge, we expect that when we change the different values of $N$ and $M$, the OPE of (6.1) still satisfies together with the corresponding central charge. The structure constants do not change and are given by function of central charge as above. Therefore, we obtain the general OPE for arbitrary $N$ and $M$ by realizing modified central charge written in terms of $N, M$ and $k$.

We claim that the OPE between the neutral spin- 3 current and itself is described as

$$
\begin{aligned}
W^{(3)}(z) W^{(3)}(w)= & \frac{1}{(z-w)^{6}} \frac{\hat{c}}{3}+\frac{1}{(z-w)^{4}} 2 \hat{T}(w)+\frac{1}{(z-w)^{3}} \partial \hat{T}(w) \\
& +\frac{1}{(z-w)^{2}}\left[\frac{3}{10} \partial^{2} \hat{T}+\frac{32}{(5 \hat{c}+22)}\left(\hat{T} \hat{T}-\frac{3}{10} \partial^{2} \hat{T}\right)+W^{(4)}\right](w) \\
& +\frac{1}{(z-w)}\left[\frac{1}{15} \partial^{3} \hat{T}+\frac{1}{2} \frac{32}{(5 \hat{c}+22)} \partial\left(\hat{T} \hat{T}-\frac{3}{10} \partial^{2} \hat{T}\right)+\frac{1}{2} \partial W^{(4)}\right](w) \\
& +\cdots,
\end{aligned}
$$

\footnotetext{
${ }^{16}$ Then we have $T(z) W^{(4)}(w)=\frac{1}{(z-w)^{2}} 4 W^{(4)}(w)+\frac{1}{(z-w)} \partial W^{(4)}(w)+\cdots$.
} 
where the modified central charge and modified stress energy tensor for generic $(N, M)$ are given by

$$
\begin{aligned}
\hat{c} & =c-\frac{k\left(M^{2}-1\right)}{(k+M)}=\frac{\left(k^{2}-1\right) M N(2 k+M+N)}{(k+M)(k+N)(k+M+N)}, \\
\hat{T}(w) & =T(w)-\frac{1}{2(k+M)} J^{a} J^{a}(w) .
\end{aligned}
$$

The central charge in (6.8) is fixed by manipulating the overall constant $b_{1}^{2}$ in the spin-3 current (2.25). Once we fix the structure constant in the fourth order pole as two, then the corresponding descendant terms with known coefficients are determined automatically. Moreover, the first order pole can be determined from the information of the second order pole. Because the additional term in the modified central charge in (6.9) under the infinity limit of $k$ contributes to $-\left(M^{2}-1\right)$ which can be ignored, the modified central charge behaves as $\hat{c} \rightarrow M\left(1-\lambda^{2}\right) k$ where $\lambda \equiv \frac{k}{(k+N)}$ [2]. The coefficient of the quadratic $J^{a} J^{a}(w)$ in (6.9) becomes $-\frac{1}{2 k}$ under the infinity limit of $k$. We expect from the experience of $[24,32]$ that the neutral primary spin- 4 current contains $d^{a b e} d^{c d e} J^{a} J^{b} J^{c} J^{d}(z)$ as well as other terms.

\section{The OPE between the charged (higher) spin currents and the uncharged higher spin-3 current}

\subsection{The OPE $J^{a}(z) W^{(3)}(w)$}

We can calculate the OPE between $J^{a}(z)$ and $W^{(3)}(w)$. The third order pole is given by $\left[2(k+M) a_{10}+N\left(a_{12}+a_{13}\right)\right] J^{a}(w)$ which vanishes by imposing the condition (2.32). Furthermore it turns out, under the condition of (2.32), that the nontrivial second order pole is

$$
\begin{aligned}
J^{a}(z) W^{(3)}(w)= & \frac{1}{(z-w)^{2}}\left[\frac{N}{2(2 k+M)}\left(-3(k+N)(k+2 N) b_{1}+M(k+2 M) b_{7}\right)\right. \\
& \left.+\frac{M}{2}\left(3(k+M) b_{2}+N b_{7}\right)\right] d^{a b c} J^{b} J^{c}(w)+\cdots
\end{aligned}
$$

According to the discussion of next subsection, the coefficients $b_{2}$ and $b_{7}$ can be determined completely in terms of $b_{1}$ and leads to the vanishing of second order pole in (7.1). Therefore, the spin- 1 current is decoupled. In other words,

$$
J^{a}(z) W^{(3)}(w)=0+\cdots
$$

In addition to the modified stress energy tensor (6.9) with (6.4) and the spin-4 current (6.5) with (6.6), this neutral spin-3 current with (7.2) belongs to the generators of $W$ algebra. 


\subsection{The OPE $K^{a}(z) W^{(3)}(w)$}

For the calculation of $b_{7}$ term in the second order pole of the $\operatorname{OPE} K^{a}(z) W^{(3)}(w)$, the following identity

$$
\operatorname{Tr}\left(t^{a} t^{b} t^{c} t^{d}\right)=\frac{1}{M} \delta^{a b} \delta^{c d}+\frac{1}{4}(i f+d)^{a b e}(i f+d)^{e c d}
$$

is used. It is rather nontrivial to calculate the OPEs between the composite operators (evaluated at $z$ ) appearing in the first order pole in the first equation of (3.1) and $\delta_{\rho \bar{\sigma}} t_{j \bar{i}}^{b} J^{(\bar{\sigma} j)}(w)$.

We focus on the particular singular terms in the second order pole. It is rather nontrivial to calculate the contributions from $b_{7}$ and $b_{8}$ terms. We can collect $J^{a} J^{u(1)} J^{u(1)}(w)$ term coming from $b_{5}, b_{7}$ and $b_{8}$ terms as follows:

$$
\begin{aligned}
& 2 M \\
& \qquad \frac{2 N}{k} \sqrt{\frac{M+N}{M N}} b_{5}-\frac{4}{k M}(k+N)(M+N) b_{7}+\frac{4}{k} N(2 k+M+2 N) \sqrt{\frac{M+N}{M N}} b_{8} \\
& =C_{K^{a} W}^{P^{a}} a_{4},
\end{aligned}
$$

where the corresponding coefficient of $P^{a}(w)$ is given by $a_{4}$ and the coefficient of $P^{a}(w)$ is denoted by $C_{K^{a} W}^{P^{a}}$ we should determine. For the $d^{a b c} J^{b} J^{c} J^{u(1)}(w)$ term coming from $b_{2}, b_{5}, b_{7}$ and $b_{8}$ terms we have

$$
\begin{aligned}
& 3 M \frac{2 N}{k} \sqrt{\frac{M+N}{M N}} b_{2}-2 M \frac{N}{(2 k+M)} b_{5}+\frac{2 N\left(4 k^{2}+2 k M+4 k N+M N\right)}{k(2 k+M)} \sqrt{\frac{M+N}{M N}} b_{7} \\
& -2 N \frac{(2 k+M+2 N)}{(2 k+M)} b_{8}=C_{K^{a} W}^{P^{a}} a_{9},
\end{aligned}
$$

where the corresponding coefficient of $P^{a}(w)$ is given by $a_{9}$ in the right hand side. Moreover, the $d^{a b c} d^{c d e} J^{b} J^{d} J^{e}(w)$ term leads to the following relation

$$
-3 M \frac{N}{(2 k+M)} b_{2}-N \frac{(2 k+M+2 N)}{(2 k+M)} b_{7}=C_{K^{a} W}^{P^{a}} \frac{3}{2} a_{17}
$$

where the corresponding coefficient of $P^{a}(w)$ is $\frac{3}{2} a_{17}$. By solving the equations (7.4), (7.5) and (7.6) together with (2.22) and (2.32), we obtain

$$
\begin{aligned}
b_{2} & =-\frac{N(k+N)(k+2 N)}{M(k+M)(k+2 M)} b_{1}, \quad b_{7}=\frac{3(k+N)(k+2 N)}{M(k+2 M)} b_{1}, \\
C_{K^{a} W}^{P^{a}} & =-\frac{12(3 k+2 M)(k+N)(k+M+N)}{M(k+2 M)} \frac{b_{1}}{a_{1}} .
\end{aligned}
$$

Then all the coefficients in the neutral spin-3 current are completely fixed. See appendix F.

From the term of $i f^{a b c} \delta_{\rho \bar{\sigma}} t_{j \bar{i}}^{c} J^{b} J^{u(1)} J^{(\rho \bar{i})} J^{\bar{\sigma} j}(w)$ associated with $a_{7}$ term of (2.14), the following relation satisfies

$$
2 a_{7} C_{K^{a} W}^{J^{b} P^{c}}=4\left[b_{5}+\sqrt{\frac{M+N}{M N}} b_{7}-b_{8}\right] .
$$


Then by substituting (2.22), (2.32) and (7.7) into (7.8), we obtain the structure constant

$$
C_{K^{a} W}^{J^{b} P^{c}}=\frac{24(k+N)(k+M+N)}{M(k+2 M)} \frac{b_{1}}{a_{1}}
$$

Also other terms can be checked. See appendix G.

Therefore, we have the following OPE between the charged spin- 2 current and the uncharged spin-3 current

$$
\begin{aligned}
K^{a}(z) W^{(3)}(w)= & -\frac{1}{(z-w)^{2}}\left[\frac{12(3 k+2 M)(k+N)(k+M+N)}{M(k+2 M)}\right] \frac{b_{1}}{a_{1}} P^{a}(w) \\
& +\frac{1}{(z-w)}\left[-\frac{1}{3} \frac{12(3 k+2 M)(k+N)(k+M+N)}{M(k+2 M)} \frac{b_{1}}{a_{1}} \partial P^{a}\right. \\
& \left.+\frac{24(k+N)(k+M+N)}{M(k+2 M)} \frac{b_{1}}{a_{1}}\left(i f^{a b c} J^{b} P^{c}+\frac{M}{3} \partial P^{a}\right)\right](w) \\
& +\cdots,
\end{aligned}
$$

where the relation (7.9) is used. Note that the last line in (7.10) is a primary operator written in terms of the known spin-1 and spin-3 currents. Compared to the one in (4.45), the OPE structure is rather simple because in this case, there exists only one free index. Under the large $k$ limit, the structure constant in the second order pole becomes $\frac{36 k^{2}}{\lambda^{2} M}$ and the one in the last line of (7.10) leads to $\frac{24 k}{\lambda^{2} M}$.

\subsection{The OPE $P^{a}(z) W^{(3)}(w)$ with $(N, M)=(5,4)$}

\subsubsection{The sixth, fifth, fourth and third order poles}

Because the free index of this OPE is given by the index $a$, there will no singular terms in sixth and fifth order poles. The nonzero singular terms appear in the fourth order pole. The natural candidate is given by the charged spin- 2 current $K^{a}(w)$. In the third order pole, there will be a quasi charged spin-3 operator in addition to the descendant term. It turns out that there exists a $i f^{a b c} J^{b} K^{c}(w)$ term with derivative term which is a primary.

\subsubsection{The second and first order poles}

The quasi charged spin- 4 current can also arise and the composite operators between the spin-1 operator and the spin-3 current with appropriate tensor structures occur. We can consider the derivative terms with free index $a$ without any difficulty. 
We summarize the OPE as follows:

$$
\begin{aligned}
& P^{a}(z) W^{(3)}(w)= \\
& \quad-\frac{1}{(z-w)^{4}}\left[\frac{9(k-2)(k+2)^{2}(k+5)(k+6)(k+9)(k+10)}{4 k(k+4)(k+8)}\right] a_{1} b_{1} K^{a}(w) \\
& +\frac{1}{(z-w)^{3}}\left[-\frac{1}{2} \frac{9(k-2)(k+2)^{2}(k+5)(k+6)(k+9)(k+10)}{4 k(k+4)(k+8)} a_{1} b_{1} \partial K^{a}\right. \\
& +\frac{9(k-2)(k+2)(k+5)(k+6)(k+9)(k+10)}{8 k(k+4)(k+8)} a_{1} b_{1} \\
& \left.\quad \times\left(i f^{a b c} J^{b} K^{c}+\frac{M}{2} \partial K^{a}\right)\right](w) \\
& +\frac{1}{(z-w)^{2}}\left[-\frac{3}{20} \frac{9(k-2)(k+2)^{2}(k+5)(k+6)(k+9)(k+10)}{4 k(k+4)(k+8)} a_{1} b_{1} \partial^{2} K^{a}\right. \\
& +\frac{1}{2} \frac{9(k-2)(k+2)(k+5)(k+6)(k+9)(k+10)}{8 k(k+4)(k+8)} a_{1} b_{1} \\
& \left.\quad \times \partial\left(i f^{a b c} J^{b} K^{c}+\frac{M}{2} \partial K^{a}\right)+R_{2}^{a}\right](w)+\mathcal{O}\left(\frac{1}{(z-w)}\right)+\cdots
\end{aligned}
$$

Note that there is a $(k+10)$ factor. A quasi primary spin-4 takes the form

$$
\begin{aligned}
R_{2}^{a}(w) \equiv & \frac{3(k+5)(k+9)}{(k+8)} b_{1}\left[\frac{(k+4)}{8} R^{a}(w)-\frac{(k+4)}{k} \frac{a_{1}}{b_{1}} J^{a} W^{(3)}(w)\right. \\
& \left.+\frac{k(k+7)(3 k+8)}{2(k+2)(k+4)} d^{a b c} J^{b} P^{c}(w)\right] \\
& +\frac{9(k+2)(k+5)(k+6)(k+9)(k+10)}{(k+4)(k+8)(3 k+8)} a_{1} b_{1}\left[\frac{k}{8(k+4)} d_{4 S S 2}^{b c d a} J^{b} J^{c} K^{d}\right. \\
& +\frac{\left(k^{2}+16\right)}{16 k(k+4)} d_{4 A A 1}^{b a c d} J^{b} J^{c} K^{d}+\frac{\left(k^{2}+6 k+16\right)}{4(k+4)(k+8)} \delta^{b a} \delta^{c d} J^{b} J^{c} K^{d} \\
& +\frac{1}{8} \delta^{b c} \delta^{d a} J^{b} J^{c} K^{d}+\frac{(k-2)\left(k^{2}+3 k-8\right)}{20 k} \partial^{2} K^{a} \\
& \left.-\frac{(k-2)(k+8)}{16 k} i f^{a b c} J^{b} \partial K^{c}-\frac{\left(k^{2}+k-16\right)}{8 k} i f^{a b c} \partial J^{b} K^{c}\right](w),
\end{aligned}
$$

where $R^{a}(w)$ is given by (4.38). Again by using the relation (4.39), we can rewrite the above in terms of quasi primary spin- 4 current. Compared to the previous OPE between the spin-3 current and itself (5.1), the OPE structure is rather simple. We expect that in the first order pole there will be no new (quasi)primary field. Although the construction in (4.45) provides the information on the quasi primary spin-4 current, due to the presence of free two indices, we should multiply the $f$ symbols into the first order pole. On the other hands, the construction in (7.11) is rather complicated because the spins of the left hand side are given by three and three. Nonetheless, due to the one single free index, 
once we have determined the second order pole, then the quasi primary spin- 4 current is determined without manipulating further. After subtracting the descendant terms, we are left with the quasi primary spin-4 current.

\section{Conclusions and outlook}

In particular, we have constructed i) the OPE between the charged spin- 2 current and itself in (3.34) with (3.35), ii) the OPE between the charged spin-2 current and the charged spin-3 current in (4.45) where the first order pole is known for $(N, M)=(5,4)$ case by rearranging it in terms of the known operators, iii) the OPE between the neutral spin-3 current and itself (6.8) where the neutral primary spin-4 current is known for $(N, M)=(5,4)$ and iv) the OPE between the charged spin-2 current and the neutral spin-3 current in (7.10).

In doing this, we have determined the charged quasi primary spin-4 current in (4.39) together with (4.38) and appendix (E.1) in terms of coset realization completely. In the OPE between the charged spin- 3 current and the neutral spin-3 current for fixed $(N, M)=$ $(5,4)$ values, we have checked that the above charged quasi primary spin- 4 current $(4.39)$ occurs at the second order pole of this OPE. We have some evidence for the presence of the above neutral primary spin- 4 current in the second order pole in the OPE between the charged spin-3 current and itself for fixed $(N, M)=(5,4)$ by focusing on the particular nontrivial term.

Under the presence of the charged higher spin currents, the algebra obtained from the whole charged and neutral higher spin currents leads to the one in an extension of [5]. The algebra coming from the neutral ones is closed. Its extension is closed and the right hand side contains the whole charged and neutral higher spin currents in general.

We list the possible open problems along the line of this paper as follows:

\section{- More OPEs}

So far, the charged spin-2,3,4 currents and the neutral spin-3 current are known in terms of coset realization. It is an open problem to determine the neutral spin- 4 current in terms of coset realization for generic $(N, M)$. Moreover, some of the OPEs we have presented in this paper do not have their complete expressions. In doing this, the new quasi primary spin-5 current will be determined. In the bulk theory side, it is an open problem to construct an extension of the higher spin algebra studied in $[33,34]$ for general $M$ by adding the $\mathrm{SO}(2 N M)$ factor in the numerator of the coset (1.1). It is better to oberve how the case $M=2$ and the case $M=4$, where the nontrivial $\mathrm{SU}(M)$ invariant tensors can occur, appear explicitly.

- Three point functions

Because the charged spin-2,3,4 currents and the neutral spin-3 current are known explicitly, it is natural to ask what are the three-point functions by evaluating the zero mode eigenvalue equations of these currents in the large $N$ limit. The relevant primary states in the coset $(1.1)$ are given by $\left(\Lambda_{N+M} ; \Lambda_{N}, m\right)$ where $\Lambda_{L}$ represents the highest weight of $\mathrm{SU}(L)$ and $m$ is the $\mathrm{U}(1)$ charge [35]. Recall that the previous 
relevant works are given in $[24,32,36-38]$ and we will keep track of the nonsinglet parts of the construction. The nontrivial part is to identify the $\mathrm{SU}(M)$ adjoint indices in the three point functions explicitly.

- Orthogonal group

So far we have considered the special unitary group in the coset model. We can apply the present results for the unitary group to the orthogonal group [39] where they decompose the $\mathrm{SU}(M)$ generators into $\frac{M(M-1)}{2}$ antisymmetric matrices and $\left(M^{2}-1\right)-\frac{M(M-1)}{2}$ traceless symmetric matrices. For the former, we do have spin1 current and for the latter, we can associate with the spin- 2 current. Then the nontrivial OPE between the spin-1 current and the spin-2 current will give us the nontrivial structure constant whose indices are mixed together at the first order pole. This will be an extension of $[40,41]$. We need to classify the various invariant tensors in this context correctly.

- Supersymmetric case

By the additional $\mathrm{SO}(2 N M)$ factor, which leads to $N M$ complex fermions, in the numerator of (1.1), the $\mathcal{N}=2$ supersymmetric model is studied in [35] where the spin contents are given by one $\mathrm{U}(1)$ spin- 1 current, two $\left(M^{2}-1\right)$ spin- 1 currents, $2 M^{2}$ spin- $s$ currents $(s=2,3, \cdots, n), M^{2}$ spin- $(n+1)$ currents and $2 M^{2}$ spin- $\left(s-\frac{1}{2}\right)$ currents $(s=2,3, \cdots,(n+1))$. Note that the standard $\mathrm{U}(1)$ spin-1 current, two spin- $\frac{3}{2}$ currents and spin- 2 stress energy tensor of $\mathcal{N}=2$ superconformal algebra can be seen from the above spin contents. It is natural to observe how the previous works in $[42,43]$ can be generalized in this enlarged model. Furthermore, for the particular level $k=N$ or $k=N+M$ [44], we expect to have the supersymmetric models and it is an open problem to observe how an extension of [45, 46] arises. See also the relevant work in [47] for different supersymmetry and there are some partial lists on the supersymmetric cases in [48-57]. Due to the complex fermions, the (higher) spin currents will contain the bosonic currents as well as these complex fermions. Moreover, it is known that under the superalgebra description on [23], we have similar coset construction. Then it is an open problem to consider the coset construction [35] where the numerator is given by the superalgebra.

\section{Acknowledgments}

We would like to thank C. Peng for the general discussion on the higher spin square [58-60] and Y. Hikida for the discussions on his papers [1, 2, 35, 44]. This work was supported by the National Research Foundation of Korea (NRF) grant funded by the Korea government (MSIT) (No. 2020R1F1A1066893). CA acknowledges warm hospitality from the School of Liberal Arts (and Institute of Convergence Fundamental Studies), Seoul National University of Science and Technology. 


\section{A An $\mathrm{SU}(M)$ invariant tensors in terms of Kronecker delta, $f$ and $d$ symbols}

Let us present the various $\mathrm{SU}(M)$ invariant tensors in terms of $f$ and $d$ symbols of rank 3

$$
\begin{aligned}
& d_{4 S S 1}^{a b c d}=\frac{4}{M} \delta^{a b} \delta^{c d}+d^{a b e} d^{e c d}, \\
& d_{4 S S 2}^{a b c d}=\frac{2}{M} \delta^{a d} \delta^{b c}+\frac{2}{M} \delta^{a c} \delta^{b d}-\frac{1}{2} f^{a c e} f^{e b d}+\frac{i}{2} f^{a c e} d^{e b d}+\frac{i}{2} d^{a c e} f^{e b d}+\frac{1}{2} d^{a c e} d^{e b d} \\
& -\frac{1}{2} f^{b c e} f^{e a d}+\frac{i}{2} f^{b c e} d^{e a d}+\frac{i}{2} d^{b c e} f^{e a d}+\frac{1}{2} d^{b c e} d^{e a d}, \\
& d_{4 S A}^{a b c d}=d^{a b e} f^{e c d}, \quad d_{4 A A 1}^{a b c d}=f^{a b e} f^{e c d}, \\
& d_{4 A A 2}^{a b c d}=\frac{2}{M} \delta^{a c} \delta^{b d}-\frac{2}{M} \delta^{a d} \delta^{b c}-\frac{1}{2} f^{a c e} f^{e b d}+\frac{1}{2} f^{a d e} f^{e b c}+\frac{i}{2} f^{a c e} d^{e b d} \\
& -\frac{i}{2} f^{a d e} d^{e b c}+\frac{i}{2} d^{a c e} f^{e b d}-\frac{i}{2} d^{a d e} f^{e b c}+\frac{1}{2} d^{a c e} d^{e b d}-\frac{1}{2} d^{a d e} d^{e b c}, \\
& d_{51}^{a b c d e}=\frac{2}{M} \delta^{f c} \delta^{d e}+\frac{2}{M} \delta^{f e} \delta^{c d}+\frac{2}{M} \delta^{f d} \delta^{c e}+\frac{i}{2} f^{f c g} d^{g d e}+\frac{1}{2} d^{f c g} d^{g d e} \\
& +\frac{i}{2} f^{f e g} d^{g c d}+\frac{1}{2} d^{f e g} d^{g c d}+\frac{i}{2} f^{f d g} d^{g c e}+\frac{1}{2} d^{f d g} d^{g c e}, \\
& d_{52}^{a b c d e}=d_{51}^{a b c d e}+i f^{c b f}\left(\frac{1}{M} \delta^{a f} \delta^{d e}+\frac{1}{4}(i f+d)^{a f g}(i f+d)^{g d e}\right) \\
& -i f^{c a f}\left(\frac{1}{M} \delta^{b f} \delta^{d e}+\frac{1}{4}(i f+d)^{b f g}(i f+d)^{g d e}\right) \\
& +i f^{c b f}\left(\frac{1}{M} \delta^{a f} \delta^{e d}+\frac{1}{4}(i f+d)^{a f g}(i f+d)^{g e d}\right) \\
& -i f^{c a f}\left(\frac{1}{M} \delta^{b f} \delta^{e d}+\frac{1}{4}(i f+d)^{b f g}(i f+d)^{g e d}\right) \\
& +i f^{e b f}\left(\frac{1}{M} \delta^{a f} \delta^{c d}+\frac{1}{4}(i f+d)^{a f g}(i f+d)^{g c d}\right) \\
& -i f^{e a f}\left(\frac{1}{M} \delta^{b f} \delta^{c d}+\frac{1}{4}(i f+d)^{b f g}(i f+d)^{g c d}\right) \\
& +i f^{d b f}\left(\frac{1}{M} \delta^{a f} \delta^{c e}+\frac{1}{4}(i f+d)^{a f g}(i f+d)^{g c e}\right) \\
& -i f^{d a f}\left(\frac{1}{M} \delta^{b f} \delta^{c e}+\frac{1}{4}(i f+d)^{b f g}(i f+d)^{g c e}\right) \\
& +i f^{d b f}\left(\frac{1}{M} \delta^{a f} \delta^{e c}+\frac{1}{4}(i f+d)^{a f g}(i f+d)^{g e c}\right) \\
& -i f^{d a f}\left(\frac{1}{M} \delta^{b f} \delta^{e c}+\frac{1}{4}(i f+d)^{b f g}(i f+d)^{g e c}\right) \\
& +i f^{e b f}\left(\frac{1}{M} \delta^{a f} \delta^{d c}+\frac{1}{4}(i f+d)^{a f g}(i f+d)^{g d c}\right) \\
& -i f^{e a f}\left(\frac{1}{M} \delta^{b f} \delta^{d c}+\frac{1}{4}(i f+d)^{b f g}(i f+d)^{g d c}\right) .
\end{aligned}
$$

We can further simplify these relations when they are multiplied by some composite operators having symmetric or antisymmetric properties in the indices. 


\section{B The first order pole in the OPE between the charged spin-2 current and itself}

\section{B.1 The substitution of charged spin-2 current}

In this appendix, we simplify (3.15) by rearranging the operators and substituting the spin-2 current (2.11) and obtain

$$
\begin{aligned}
& i f^{a b c} N \partial K^{c}(w)=i f^{a b c} N \partial\left[\delta_{\rho \bar{\sigma}} t_{j \bar{i}}^{c}\left(J^{(\rho \bar{i})} J^{(\bar{\sigma} j)}+J^{(\bar{\sigma} j)} J^{(\bar{\rho} \bar{i})}\right)-\frac{N}{(M+2 k)} d^{c f g} J^{f} J^{g}\right. \\
& \left.+\frac{2 N}{k} \sqrt{\frac{M+N}{M N}} J^{c} J^{u(1)}\right](w) \\
& i f^{a c e} d^{b c d} K^{e} J^{d}(w)=i f^{a c e} d^{b c d} J^{d}\left[\delta_{\rho \bar{\sigma}} t_{j \bar{i}}^{e}\left(J^{(\rho \bar{i})} J^{(\bar{\sigma} j)}+J^{(\bar{\sigma} j)} J^{(\bar{\rho} \bar{i})}\right)-\frac{N}{(M+2 k)} d^{e f g} J^{f} J^{g}\right. \\
& \left.+\frac{2 N}{k} \sqrt{\frac{M+N}{M N}} J^{e} J^{u(1)}\right](w)+M d^{a b c} \partial\left[\delta_{\rho \bar{\sigma}} t_{j \bar{i}}^{c}\left(J^{(\bar{\rho} \bar{i})} J^{(\bar{\sigma} j)}+J^{(\bar{\sigma} j)} J^{(\rho \bar{i})}\right)\right. \\
& \left.-\frac{N}{(M+2 k)} d^{c f g} J^{f} J^{g}+\frac{2 N}{k} \sqrt{\frac{M+N}{M N}} J^{c} J^{u(1)}\right](w), \\
& i f^{a d e} d^{b c d} J^{c} K^{e}=i f^{a d e} d^{b c d} J^{c}\left[\delta_{\rho \bar{\sigma}} t_{j \bar{i}}^{e}\left(J^{(\rho \bar{i})} J^{(\bar{\sigma} j)}+J^{(\bar{\sigma} j)} J^{(\bar{\rho} \bar{i})}\right)-\frac{N}{(M+2 k)} d^{e f g} J^{f} J^{g}\right. \\
& \left.+\frac{2 N}{k} \sqrt{\frac{M+N}{M N}} J^{e} J^{u(1)}\right](w) \\
& i f^{a b c} K^{c} J^{u(1)}(w)=i f^{a b c} J^{u(1)}\left[\delta_{\rho \bar{\sigma}} t_{j \bar{i}}^{c}\left(J^{(\rho \bar{i})} J^{(\bar{\sigma} j)}+J^{(\bar{\sigma} j)} J^{(\rho \bar{i})}\right)\right. \\
& \left.-\frac{N}{(M+2 k)} d^{c f g} J^{f} J^{g}+\frac{2 N}{k} \sqrt{\frac{M+N}{M N}} J^{c} J^{u(1)}\right](w), \\
& \delta_{\rho \bar{\sigma}} t_{j \bar{i}}^{b} \delta^{k \bar{i}} t_{k \bar{l}}^{a}\left(\left(J^{u(1)} J^{(\rho \bar{l})}\right) J^{(\bar{\sigma} j)}\right)(w)=\left[\frac{k N}{2} \delta^{a b} \partial^{2} J^{u(1)}\right. \\
& -\sqrt{\frac{M+N}{M N}}\left(\frac{1}{M} \delta^{a b} \delta_{j \bar{i}}+\frac{1}{2}(i f+d)^{b a c} t_{j \bar{i}}^{c}\right) \delta_{\rho \bar{\sigma}} \partial J^{(\rho \bar{i})} J^{(\bar{\sigma} j)} \\
& +N \sqrt{\frac{M+N}{M N}} \partial J^{u(1)} J^{u(1)}+\frac{i}{2} N f^{a b c} J^{c} \partial J^{u(1)}-\frac{N}{2} d^{a b c} J^{c} \partial J^{u(1)} \\
& \left.+\delta_{\rho \bar{\sigma}}\left(\frac{1}{M} \delta^{b a} \delta_{j \bar{i}}+\frac{1}{2}(i f+d)^{b a c} t_{j \bar{i}}^{c}\right) J^{u(1)} J^{(\rho \bar{i})} J^{(\bar{\sigma} j)}\right](w), \\
& \delta_{\rho \bar{\sigma}} t_{j \bar{i}}^{b}\left(\left(J^{a} J^{(\rho \bar{i})}\right) J^{(\bar{\sigma} j)}\right)(w)=\left(\frac{1}{M} \delta^{a b} \delta_{j \bar{i}}+\frac{1}{2}(i f+d)^{a b c} t_{j \bar{i}}^{c}\right) \delta_{\rho \bar{\sigma}} \partial J^{(\rho \bar{i})} J^{(\bar{\sigma} j)} \\
& \left.-N \partial J^{a} J^{b}+\delta_{\rho \bar{\sigma}} t_{j \bar{i}}^{b} J^{a} J^{(\rho \bar{i})} J^{(\bar{\sigma} j)}\right](w),
\end{aligned}
$$




$$
\begin{aligned}
& \delta_{\rho \bar{\sigma}} t_{j \bar{i}}^{b}\left(i f-\frac{(2 k+M+2 N)}{(2 k+M)} d\right)^{a c d} \delta^{k \bar{k}} t_{k \bar{l}}^{d}\left(\left(J^{c} J^{(\rho \bar{l})}\right) J^{(\bar{\sigma} j)}\right)(w)=\left[-\frac{1}{2} k N i f^{a b c} \partial^{2} J^{c}\right. \\
& -\frac{k N(2 k+M+2 N)}{2(2 k+M)} d^{a b c} \partial^{2} J^{c}-\frac{2\left(-4 k-2 M-4 N+M^{2} N\right)}{M^{2}(2 k+M)} \delta^{a b} \delta_{\rho \bar{\sigma}} \delta_{j \bar{i}} \partial J^{(\rho \bar{i})} J^{(\bar{\sigma} j)} \\
& +\frac{(2 N+M+2 k)}{(2 k+M)} \frac{2}{M} d^{a b c} \delta_{\rho \bar{\sigma}} t_{j \bar{i}}^{c} \partial J^{(\bar{\rho} \bar{i})} J^{(\bar{\sigma} j)}-\sqrt{\frac{M+N}{M N}} N f^{a b c} \partial J^{c} J^{u(1)} \\
& -\frac{(2 N+M+2 k)}{(2 k+M)} \sqrt{\frac{M+N}{M N}} N d^{a b c} \partial J^{c} J^{u(1)} \\
& -\frac{N}{2}\left(i f-\frac{(2 k+M+2 N)}{(2 k+M)} d\right)^{a c d}(i f+d)^{e b d} \partial J^{c} J^{e} \\
& \left.+\left(i f-\frac{(2 k+M+2 N)}{(2 k+M)} d\right)^{a c d}\left(\frac{1}{M} \delta^{b d} \delta_{j \bar{i}}+\frac{1}{2}(i f+d)^{b d e} t_{j \bar{j}}^{e}\right) \delta_{\rho \bar{\sigma}} J^{c} J^{(\rho \bar{i})} J^{(\bar{\sigma} j)}\right](w), \\
& \delta_{\rho \bar{\sigma}} t_{j \bar{i}}^{b} \bar{i}^{k \bar{i}} \delta_{\sigma_{1} \overline{\sigma_{1}}}\left(t^{\alpha}\right)^{\overline{\sigma_{1}} \rho} t_{k \bar{l}}^{a}\left(\left(J^{\alpha} J^{\left(\sigma_{1} \bar{l}\right)}\right) J^{(\bar{\sigma} j)}\right)(w)= \\
& {\left[\left(t^{\alpha}\right)^{\sigma \bar{\rho}} J^{\alpha} J^{(\sigma \bar{i})} J^{(\bar{\rho} j)}\left(\frac{1}{M} \delta^{a b} \delta_{j \bar{i}}+\frac{1}{2}(i f+d)^{a b c} t_{j \bar{i}}^{c}\right)\right.} \\
& \left.-\frac{\left(N^{2}-1\right)}{N}\left(\frac{1}{M} \delta^{a b} \delta_{j \bar{i}}+\frac{1}{2}(i f+d)^{b a c} t_{j \bar{i}}^{c}\right) \delta_{\rho \bar{\sigma}} \partial J^{(\rho \bar{i})} J^{(\bar{\sigma} j)}+\delta^{a b} \partial J^{\alpha} J^{\alpha}\right](w), \\
& \delta_{\rho \bar{\sigma}} t_{j \bar{i}}^{b} \delta_{k \bar{l}}\left(t^{a}\right)^{\bar{l} j} J^{(\bar{\rho} \bar{i})} \partial J^{(\bar{\sigma} k)}(w)=\delta_{\rho \bar{\sigma}}\left(\frac{1}{M} \delta^{a b} \delta_{j \bar{i}}+\frac{1}{2}(i f+d)^{a b c} t_{j \bar{i}}^{c}\right) J^{(\rho \bar{i})} \partial J^{(\bar{\sigma} i)}(w), \\
& \delta_{\rho \bar{\sigma}} t_{j \bar{i}}^{b} \delta^{j \bar{l}} t_{k \bar{l}}^{a} J^{(\rho \bar{i})} J^{u(1)} J^{(\bar{\sigma} k)}(w)=\left[\delta_{\rho \bar{\sigma}}\left(\frac{1}{M} \delta^{a b} \delta_{j \bar{i}}+\frac{1}{2}(i f+d)^{a b c} t_{j \bar{i}}^{c}\right) J^{u(1)} J^{(\rho \bar{i})} J^{(\bar{\sigma} j)}\right. \\
& \left.-\sqrt{\frac{M+N}{M N}} \delta_{\rho \bar{\sigma}}\left(\frac{1}{M} \delta^{a b} \delta_{j \bar{i}}+\frac{1}{2}(i f+d)^{a b c} t_{j \bar{i}}^{c}\right) \partial J^{(\rho \bar{i})} J^{(\bar{\sigma} j)}\right](w),
\end{aligned}
$$

$\delta_{\rho \bar{\sigma}} t_{j \bar{i}}^{b} J^{(\rho \bar{i})} J^{a} J^{(\bar{\sigma} j)}(w)=\left[\delta_{\rho \bar{\sigma}} t_{j \bar{i}}^{b} J^{a} J^{(\rho \bar{i})} J^{(\bar{\sigma} j)}\right.$

$$
\begin{gathered}
\left.+\delta_{\rho \bar{\sigma}}\left(\frac{1}{M} \delta^{a b} \delta_{j \bar{i}}+\frac{1}{2}(i f+d)^{b a c} t_{j \bar{i}}^{c}\right) \partial J^{(\bar{\rho} \bar{i})} J^{(\bar{\sigma} j)}\right](w), \\
\delta_{\rho \bar{\sigma}} t_{j \bar{i}}^{b}\left(i f+\frac{(2 k+M+2 N)}{(2 k+M)} d\right)^{a c d} \delta^{j \bar{j}_{1}} t_{k \overline{j_{1}}}^{d} J^{(\bar{\rho} \bar{i})} J^{c} J^{(\bar{\sigma} k)}(w)=
\end{gathered}
$$$$
\left[\left(i f+\frac{(2 k+M+2 N)}{(2 k+M)} d\right)^{a c d} \delta_{\rho \bar{\sigma}}\left(\frac{1}{M} \delta^{d b} \delta_{j \bar{i}}+\frac{1}{2}(i f+d)^{d b e} t_{j \bar{i}}^{e}\right) J^{c} J^{(\rho \bar{i})} J^{(\bar{\sigma} i)}\right.
$$$$
+\left(i f+\frac{(2 k+M+2 N)}{(2 k+M)} d\right)^{a c d} \delta_{\rho \bar{\sigma}}
$$$$
\left.\times\left(\frac{1}{M} \delta^{b c} t_{j \bar{i}}^{d}+\frac{1}{2 M} \delta^{d e}(i f+d)^{b c e} \delta_{j \bar{i}}+\frac{1}{4}(i f+d)^{b c e}(i f+d)^{d e f} t_{j \bar{i}}^{f}\right) \partial J^{(\rho \bar{i})} J^{(\bar{\sigma} j)}\right](w),
$$ 


$$
\begin{aligned}
& \delta_{\rho \bar{\sigma}} t_{j \bar{i}}^{b} \delta^{j \bar{l}} \delta_{\sigma_{1} \bar{\sigma}_{1}}\left(t^{\alpha}\right)^{\bar{\sigma} \sigma_{1}} t_{k \bar{l}}^{a} J^{(\rho \bar{i})} J^{\alpha} J^{\left(\overline{\sigma_{1}} k\right)}(w)= \\
& {\left[\left(t^{\alpha}\right)^{\rho \bar{\sigma}}\left(\frac{1}{M} \delta^{a b} \delta_{j \bar{i}}+\frac{1}{2}(i f+d)^{a b c} t_{j \bar{i}}^{c}\right) J^{\alpha} J^{(\rho \bar{i})} J^{(\bar{\sigma} k)}\right.} \\
&\left.-\left(N-\frac{1}{N}\right)\left(\frac{1}{M} \delta^{a b} \delta_{j \bar{i}}+\frac{1}{2}(i f+d)^{a b c} t_{j \bar{i}}^{c}\right) \delta_{\rho \bar{\sigma}} \delta_{j \bar{i}} \partial J^{(\rho \bar{i})} J^{(\bar{\sigma} j)}\right](w) .
\end{aligned}
$$

The quadratic terms in the Kronecker delta, $f$ or $d$ symbols appearing in the second relation from the last in appendix (B.1) can be simplified further.

\section{B.2 The adjoint spin-1 dependent terms in the first order pole}

Now we collect the adjoint spin-1 dependent terms only from appendix (B.1) and (3.15) in the first order pole as follows:

$$
\begin{aligned}
i f^{a b c} N \partial\left[N \partial J^{c}-\frac{N}{(M+2 k)} d^{c f g} J^{f} J^{g}\right](w) \\
-\frac{N}{(M+2 k)}\left(i f^{a c e} d^{b c d} J^{d}\left[N \partial J^{e}-\frac{N}{(M+2 k)} d^{e f g} J^{f} J^{g}\right]\right. \\
\left.+M d^{a b c} \partial\left[N \partial J^{c}-\frac{N}{(M+2 k)} d^{c f g} J^{f} J^{g}\right]\right)(w) \\
-\frac{N}{(M+2 k)}\left(i f^{a d e} d^{b c d} J^{c}\left[N \partial J^{e}-\frac{N}{(M+2 k)} d^{e f g} J^{f} J^{g}\right]\right)(w) \\
+\frac{4}{k M}(k+M+N)(-N) \partial J^{a} J^{b}(w)-2\left[-\frac{1}{2} k N i f^{a b c} \partial^{2} J^{c}\right. \\
-\frac{k N(2 k+M+2 N)}{2(2 k+M)} d^{a b c} \partial^{2} J^{c} \\
\left.-\frac{N}{2}\left(i f-\frac{(2 k+M+2 N)}{(2 k+M)} d\right)^{a c d}(i f+d)^{e b d} \partial J^{c} J^{e}\right](w),
\end{aligned}
$$

which should be equal to the terms of the first order pole in (3.34) by putting all the other spin-1 currents to zero. The cubic terms in appendix (B.2) will participate in the various places in the first order pole of (3.34). In the calculations of (3.23), (3.25) and (3.27), the above relation appendix (B.2) is used. 


\section{B.3 The first order pole}

Eventually we obtain the first order pole as follows:

$$
\begin{aligned}
& \left.K^{a}(z) K^{b}(w)\right|_{\frac{1}{(z-w)}}=-\frac{2 N}{(M+2 k)} i f^{a c e} d^{b c d} J^{d} K^{e}(w)-\frac{M N}{(M+2 k)} d^{a b c} \partial K^{c}(w) \\
& +N i f^{a b c} \partial K^{c}(w)+\frac{2 N}{k} \sqrt{\frac{M+N}{M N}} i f^{a b c} J^{u(1)} K^{c}(w)+4(k+N) \delta_{\rho \bar{\sigma}}\left(\frac{1}{M} \delta^{a b} \delta_{j \bar{i}}\right. \\
& \left.+\frac{1}{2}(i f+d)^{a b c} t_{j \bar{i}}^{c}\right) J^{(\rho \bar{i})} \partial J^{(\bar{\sigma} j)}(w) \\
& -2(k+N) N \sqrt{\frac{M+N}{M N}} \delta^{a b} \partial^{2} J^{u(1)}(w)+\frac{4(k+N)}{M} \delta^{a b} \delta_{\rho \bar{\sigma}} \delta_{j \bar{i}} \partial J^{(\rho \bar{i})} J^{(\bar{\sigma} j)}(w) \\
& -2(k+3 N) i f^{a b c} t_{j \bar{i}}^{c} \delta_{\rho \bar{\sigma}} \partial J^{(\bar{\rho} \bar{i})} J^{(\bar{\sigma} j)}(w)+2(k+N) d^{a b c} t_{j \bar{i}}^{c} \delta_{\rho \bar{\sigma}} \partial J^{(\rho \bar{i})} J^{(\bar{\sigma} j)}(w) \\
& -\frac{4(k+N)}{k} \frac{M+N}{M} \delta^{a b} \partial J^{u(1)} J^{u(1)}(w)-\frac{2 N(k+N)}{k} \sqrt{\frac{M+N}{M N}} i f^{a b c} \partial J^{u(1)} J^{c}(w) \\
& +\frac{2 N(k+N)}{k} \sqrt{\frac{M+N}{M N}} d^{a b c} \partial J^{u(1)} J^{c}(w) \\
& +\frac{4(k+N)}{k} \sqrt{\frac{M+N}{M N}} i f^{a b c} t_{j \bar{i}}^{c} \delta_{\rho \bar{\sigma}} J^{u(1)} J^{(\bar{\rho} \overline{)})} J^{(\bar{\sigma} j)}(w) \\
& -\frac{4 N(k+M+N)}{k M} \partial J^{a} J^{b}(w)+k N i f^{a b c} \partial^{2} J^{c}(w)+2 N \sqrt{\frac{M+N}{M N}} i f^{a b c} J^{u(1)} \partial J^{c}(w) \\
& +\frac{2 N(2 k+M+2 N)}{2 k+M} \sqrt{\frac{M+N}{M N}} d^{a b c} J^{u(1)} \partial J^{c}(w)+4 i f^{a b c} t_{\rho \bar{\sigma}}^{\alpha} t_{j \bar{i}}^{c} J^{\alpha} J^{(\rho \bar{i})} J^{(\bar{\sigma} j)}(w) \\
& -4 \delta^{a b} \partial J^{\alpha} J^{\alpha}(w)+\frac{k N(2 k+M+2 N)}{(2 k+M)} d^{a b c} \partial^{2} J^{c}(w) \\
& -4\left(\frac{i}{M} f^{a c b} \delta_{j \bar{i}}+\frac{i}{2} f^{a c d} d^{b d e} t_{j \bar{i}}^{e}-\frac{(2 k+M+2 N)}{2(2 k+M)} d^{a c d} i f^{b d e} t_{j \bar{i}}^{e}\right) \delta_{\rho \bar{\sigma}} J^{c} J^{(\bar{\rho} \bar{i})} J^{(\bar{\sigma} j)}(w) \\
& +N\left(i f-\frac{(2 k+M+2 N)}{(2 k+M)} d\right)^{a c d}(i f+d)^{e b d} \partial J^{c} J^{e}(w) \text {. }
\end{aligned}
$$

It is rather nontrivial to rewrite this in terms of the known currents as well as the charged spin-3 current. This can be written in terms of various quasi primary operators and the charged spin-3 current as well as the descendant terms in (3.34). The expression in appendix (B.3) will be used in the second order pole of the OPE between the charged spin-2 current and the charged spin-3 current. 


\section{The structure constants in the infinity limit of $k$ of section 3}

The structure constants appearing in (3.34) under the infinity limit of $k$ become

$$
\begin{aligned}
c_{1} & \rightarrow \frac{4\left(1-\lambda^{2}\right)}{\lambda^{2}} k^{3}, & c_{2} & \rightarrow \frac{2\left(1-\lambda^{2}\right)}{\lambda^{2}} k^{2}, & \frac{a_{1, C H} c_{1}}{c} & \rightarrow \frac{4}{\lambda^{2} M} k^{2}, \\
c_{6} & \rightarrow \frac{1}{\lambda} k, & c_{31} & \rightarrow-\frac{4}{\lambda^{2} M} k, & c_{32} & \rightarrow \frac{\left(1-\lambda^{2}\right)}{2 \lambda^{2}} k, \\
c_{33} & \rightarrow \frac{\left(1-\lambda^{2}\right)}{2 \lambda^{2}} k, & c_{34} & \rightarrow-\frac{2\left(1-\lambda^{2}\right)}{\lambda^{2}}, & c_{2} a_{3, C H} & \rightarrow \frac{8}{\lambda^{2} M} k, \\
c_{41} & \rightarrow \frac{\left(1-\lambda^{2}\right)}{12 \lambda^{2}} k, & c_{43} & \rightarrow-\frac{\left(1-\lambda^{2}\right) M}{2 \lambda^{2}} \frac{1}{k}, & c_{51} & \rightarrow-\frac{4 i}{\lambda^{2} M}, \\
c_{52} & \rightarrow \frac{\left(1-\lambda^{2}\right)}{18 \lambda^{2}}, & c_{53} & \rightarrow-\frac{\left(1-\lambda^{2}\right)}{6 \lambda^{2}}, & c_{72} & \rightarrow-\frac{1}{\lambda},
\end{aligned}
$$

together with

$$
a_{1, C H} \rightarrow 1, \quad a_{2, C H} \rightarrow \frac{1}{6}, \quad a_{3, C H} \rightarrow \frac{4}{\left(1-\lambda^{2}\right) M} \frac{1}{k}, \quad c \rightarrow\left(1-\lambda^{2}\right) M k .
$$

It is not clear whether there are some relations between these structure constants appendix (C.1) and appendix (C.2) and the ones in the free field realization given in appendix $\mathrm{H}$. Because the structure constants are given by the three parameters, we can take any limits among these. For example, the infinity limit of $N$ can be taken for fixed $\lambda$ and $M$.

\section{The second order pole in the OPE $K^{a}(z) P^{b}(w)$}

The second order pole of the OPE between the charged spin- 2 current and the charged spin-3 current can be described as

$$
\begin{aligned}
& \left.K^{a}(z) P^{b}(w)\right|_{\frac{1}{(z-w)^{2}}}=\left[\frac{2}{M}(2 k+N) \delta^{a b} \delta_{j \bar{i}} t_{\rho \bar{\sigma}}^{\alpha} J^{\alpha} J^{(\rho \bar{i})} J^{(\bar{\sigma} j)}-2 k \delta^{a b} J^{\alpha} \partial J^{\alpha}\right. \\
& +\frac{2 k(2 k+M+N)}{(2 k+M)} d^{a b c} t_{j \bar{i}}^{c} t_{\rho \bar{\sigma}}^{\alpha} J^{\alpha} J^{(\rho \bar{i})} J^{(\bar{\sigma} j)} \\
& -\frac{2}{k}(2 k+N) \sqrt{\frac{M+N}{M N}} \delta^{a b} J^{\alpha} J^{\alpha} J^{u(1)}+i f^{\alpha \beta \gamma} \delta^{a b} J^{\alpha} J^{\beta} J^{\gamma} \\
& \left.+\frac{2(2 k+M+N)}{(2 k+M)} d^{a b c} J^{\alpha} J^{\alpha} J^{c}-d^{\alpha \beta \gamma} \delta^{a b} J^{\alpha} J^{\beta} J^{\gamma}\right](w) a_{1} \\
& +\left(2 M K^{a} J^{b}-2 f^{a c d} f^{d b e} J^{c} K^{e}\right)(w) a_{3} \\
& +\left[i f^{a d e} d^{b c d}\left(K^{e} K^{c}\right)_{\text {pole- } 1}+d^{b c d} J^{d}\left(K^{a} K^{c}\right)_{\text {pole-2 }}\right. \\
& -\frac{2 N M}{k} \sqrt{\frac{M+N}{M N}} d^{a b c} K^{c} J^{u(1)}+\frac{N}{(2 k+M)}\left(i f^{a b c}\left(M^{2}-4\right) \partial K^{c}\right. \\
& -\frac{8\left(4-M^{2}\right)}{M^{2}}\left(J^{b} K^{a}-J^{a} K^{b}\right)-\frac{\left(8-M^{2}\right)}{M} d^{a c e} d^{b d e}\left(J^{d} K^{c}\right.
\end{aligned}
$$




$$
\begin{aligned}
& \left.\left.\left.-J^{c} K^{d}\right)+M d^{a b e} d^{e c d} J^{c} K^{d}+M d^{a c e} d^{b d e} J^{d} K^{c}\right)\right](w) a_{5} \\
& +\left[J^{u(1)}\left(K^{a} K^{b}\right)_{p o l e-2}+\frac{N M}{(2 k+M)} d^{a b c} J^{u(1)} K^{c}\right](w) a_{7} \\
& +\left(2(2 k+2 N+M) J^{b} K^{a}+2 i f^{a b c}(k+N) \partial K^{c}\right. \\
& \left.+2 f^{a b c} f^{c d e} J^{d} K^{e}\right)(w) a_{8} \\
& +M d^{a b c} J^{u(1)} K^{c}(w) a_{9} \\
& -f^{a c d} f^{b c e} K^{d} J^{e}(w) a_{11} \\
& +\left(\frac{2\left(k^{2}-1\right)(2 k+M+N)}{k(2 k+M)} \delta_{\rho \bar{\sigma}}\left(\frac{2}{M} \delta^{a b} \delta_{j \bar{i}}+d^{a b c} t_{j \bar{i}}^{c}\right) J^{(\rho \bar{i})} \partial J^{(\bar{\sigma} j)}\right. \\
& +\delta_{\rho \bar{\sigma}} t_{j \bar{i}}^{b}\left(\left(K^{a} J^{(\rho \bar{i})}\right)_{\text {pole- } 1} \partial J^{(\bar{\sigma} j)}\right)_{\text {pole-1 }} \\
& \left.+\delta_{\rho \bar{\sigma}} t_{j \bar{i}}^{b} J^{(\rho \bar{i})}\left(K^{a} \partial J^{(\bar{\sigma} j)}\right)_{p o l e-2}+N i f^{a b c} \partial K^{c}\right)(w)\left(a_{13}-a_{12}\right) \\
& +\left[\frac{1}{2} \partial\left(K^{a} K^{b}\right)_{\text {pole-2 }}+\frac{1}{2}\left(K^{a} K^{b}\right)_{\text {pole- } 1}\right. \\
& -\frac{N}{k} \sqrt{\frac{M+N}{M N}} i f^{a b c} K^{c} J^{u(1)}+\frac{N}{2(2 k+M)}\left(M d^{a b c} \partial K^{c}\right. \\
& \left.\left.+i f^{a d e} d^{b d f} K^{e} J^{f}+i f^{a d e} d^{b f d} J^{f} K^{e}\right)\right](w) a_{12} \\
& +2 i f^{a b c} \partial K^{c}(w) a_{16} \\
& +\left[\frac{6\left(4-M^{2}\right)}{M^{2}} J^{a} K^{b}-\frac{6}{M} f^{a b c} f^{c d e} J^{d} K^{e}+3\left(8-\frac{16}{M^{2}}\right) J^{b} K^{a}\right. \\
& -\frac{6}{M} f^{a c e} f^{b d e} J^{c} K^{d}+\frac{M}{4} i f^{c d e} d^{a b c} J^{e} K^{d}-\frac{M}{4} i f^{a b c} d^{c d e} J^{e} K^{d} \\
& +\frac{M}{4} i f^{e b d} d^{a c e} J^{d} K^{c}-\frac{M}{4} i f^{a c e} d^{e b d} J^{d} K^{c}+\frac{6\left(4-M^{2}\right)}{M^{2}} \delta^{a b} J^{c} K^{c} \\
& +3\left(M-\frac{M}{4}\right) d^{a c e} d^{e b d} J^{d} K^{c}+\frac{6}{M} d^{a c e} d^{e b d} J^{c} K^{d} \\
& \left.+\frac{6}{M} d^{a b e} d^{e c d} J^{c} K^{d}+\left(M^{2}+6\right) i f^{a b c} \partial K^{c}\right](w) a_{17} .
\end{aligned}
$$

We will further simplify these expressions in next subsections.

\section{D.1 The $a_{5}$ terms of the second order pole}

In the $a_{5}$ terms, we have the following results

$$
i f^{a d e} d^{b c d}\left(K^{e} K^{c}\right)_{\text {pole- } 1}(w)=\frac{8\left(4-M^{2}\right) N}{M^{2}(2 k+M)} J^{b} K^{a}(w)-\frac{8\left(4-M^{2}\right) N}{M^{2}(2 k+M)} J^{a} K^{b}(w)
$$




$$
\begin{aligned}
& +\frac{\left(8-M^{2}\right) N}{M(2 k+M)} d^{a c e} d^{e b d} J^{d} K^{c}(w)-\frac{\left(8-M^{2}\right) N}{M(2 k+M)} d^{a c e} d^{e b d} J^{c} K^{d}(w) \\
& -\frac{M N}{(2 k+M)} d^{a b e} d^{e c d} J^{c} K^{d}(w)-\frac{\left(M^{2}-4\right) N}{(2 k+M)} i f^{a b c} \partial K^{c}(w) \\
& +2 M(k+N) d^{a b c} \delta_{\rho \bar{\sigma}} t_{j \bar{i}}^{c} J^{(\rho \bar{i})} \partial J^{(\bar{\sigma} j)}(w) \\
& +\frac{2 M N}{k} \sqrt{\frac{M+N}{M N}} d^{a b c} J^{u(1)} K^{c}(w)+\frac{2}{M}\left(M^{2}-4\right)(k+N) i f^{a b c} \delta_{\rho \bar{\sigma}} t_{\bar{j} \bar{i}}^{c} J^{(\bar{\rho} \bar{i})} \partial J^{(\bar{\sigma} j)}(w) \\
& -2 M(k+3 N) d^{a b c} \delta_{\rho \bar{\sigma}} t_{j \bar{i}}^{c} \partial J^{(\rho \bar{i})} J^{(\bar{\sigma} j)}(w) \\
& +\frac{2}{M}\left(M^{2}-4\right)(k+N) i f^{a b c} \delta_{\rho \bar{\sigma}} t_{j \bar{i}}^{c} \partial J^{(\rho \bar{i})} J^{(\bar{\sigma} j)}(w) \\
& -\frac{2 M N(k+N)}{k} \sqrt{\frac{M+N}{M N}} d^{a b c} \partial J^{u(1)} J^{c}(w) \\
& +\frac{2\left(M^{2}-4\right) N(k+N)}{k M} \sqrt{\frac{M+N}{M N}} i f^{a b c} \partial J^{u(1)} J^{c}(w) \\
& +\frac{4 M(k+N)}{k} \sqrt{\frac{M+N}{M N}} d^{a b c} \delta_{\rho \bar{\sigma}} t_{j \bar{i}}^{c} J^{u(1)} J^{(\rho \bar{i})} J^{(\bar{\sigma} j)}(w) \\
& +\frac{N\left(4 k^{2}-k M^{2} N-6 k M+4 k N-4 M^{2}-4 M N\right)}{k M(2 k+M)} i f^{a e c} d^{b e d} \partial J^{c} J^{d}(w) \\
& +k M N d^{a b c} \partial^{2} J^{c}(w)+2 M N \sqrt{\frac{M+N}{M N}} d^{a b c} J^{u(1)} \partial J^{c}(w) \\
& +\frac{2\left(M^{2}-4\right) N(2 k+M+2 N)}{M(2 k+M)} \sqrt{\frac{M+N}{M N}} i f^{a b c} J^{u(1)} \partial J^{c}(w) \\
& +\frac{M N^{2}}{2 k+M} i d^{a c e} f^{e d b} \partial J^{c} J^{d}(w) \\
& +\frac{4\left(4-M^{2}\right) N}{M^{2}} \partial J^{a} J^{b}(w)-\frac{4\left(4-M^{2}\right) N}{M^{2}} \partial J^{b} J^{a}(w) \\
& +N\left(\frac{M(2 k+M+2 N)}{2(2 k+M)}+\frac{\left(8-M^{2}\right)}{2 M}\right) d^{a c e} d^{e d b} \partial J^{c} J^{d}(w) \\
& +\left(-\frac{M(2 k+M+2 N)}{2(2 k+M)}-\frac{8-M^{2}}{2 M}\right) N d^{a d e} d^{e c b} \partial J^{c} J^{d}(w)+\frac{M N^{2}}{(2 k+M)} d^{a b e} d^{e c d} \partial J^{c} J^{d}(w) \\
& +\frac{2 N(2 k+M+2 N)}{M(2 k+M)} i f^{a b e} d^{e c d} \partial J^{c} J^{d}(w)-\frac{2 N(2 k+M+2 N)}{M(2 k+M)} i f^{a d e} d^{e c b} \partial J^{c} J^{d}(w) \\
& +4 M t_{\rho \bar{\sigma}}^{\alpha} t_{j \bar{i}}^{c} J^{\alpha} J^{(\bar{\rho})} J^{(\bar{\sigma} j)}(w)+\frac{k\left(M^{2}-4\right) N(2 k+M+2 N)}{M(2 k+M)} i f^{a b c} \partial^{2} J^{c}(w) \\
& +M N d^{a b c} \partial K^{c}(w) \\
& +4 \delta_{\rho \bar{\sigma}} \delta_{j \bar{i}} d^{a b c} J^{c} J^{(\bar{\rho} \bar{i})} J^{(\bar{\sigma} j)}(w)-\frac{8\left(4-M^{2}\right)}{M^{2}} t_{j \bar{i}}^{b} \delta_{\rho \bar{\sigma}} J^{a} J^{(\rho \bar{i})} J^{(\bar{\sigma} j)}(w) \\
& -\frac{8\left(4-M^{2}\right)}{M^{2}} t_{j \bar{i}}^{a} \delta_{\rho \bar{\sigma}} J^{b} J^{(\rho \bar{i})} J^{(\bar{\sigma} j)}(w)
\end{aligned}
$$




$$
\begin{aligned}
& -4\left(\frac{M(2 k+M+2 N)}{4(2 k+M)}+\frac{\left(8-M^{2}\right)}{4 M}\right) \delta_{\rho \bar{\sigma}} t_{j \bar{i}}^{d} d^{a c e} d^{e d b} J^{c} J^{(\rho \bar{i})} J^{(\bar{\sigma} j)}(w) \\
& +4\left(\frac{M(2 k+M+2 N)}{4(2 k+M)}+\frac{\left(8-M^{2}\right)}{4 M}\right) \delta_{\rho \bar{\sigma}} t_{j \bar{i}}^{d} d^{a d e} d^{e c b} J^{c} J^{(\rho \bar{\rho})} J^{(\bar{\sigma} j)}(w) \\
& -\frac{2 M N}{2 k+M} \delta_{\rho \bar{\sigma}} t_{j \bar{j}}^{d} d^{a b e} d^{e c d} J^{c} J^{(\rho \bar{i})} J^{(\bar{\sigma} j)}(w) .
\end{aligned}
$$

We should analyze these complicated results in order to rewrite them in terms of the known currents. Moreover, we have the following expression

$$
\begin{aligned}
& d^{b c d} J^{d}\left(K^{a} K^{c}\right)_{p o l e-2}(w)=-\frac{4(k+N)(M+N)}{k M} d^{a b c} J^{u(1)} J^{u(1)} J^{c}(w) \\
& -4 d^{a b c} J^{\alpha} J^{\alpha} J^{c}(w)-\frac{2 N^{2}}{k} \sqrt{\frac{M+N}{M N}} i f^{a e c} d^{b e d} J^{d} J^{c} J^{u(1)}(w) \\
& +\frac{2 N\left(4 k^{2}+2 k M+4 k N+M N\right)}{k(2 k+M)} \sqrt{\frac{M+N}{M N}} d^{a e c} d^{b e d} J^{d} J^{c} J^{u(1)}(w) \\
& -\frac{4 N(k+M+N)}{k M} d^{b c d} J^{d} J^{a} J^{c}(w) \\
& +N d^{b f g}\left(i f-\frac{(2 N+M+2 k)}{(M+2 k)}\right)^{a c d}(i f+d)^{e f d} J^{g} J^{c} J^{e}(w) \\
& +\frac{8(k+N)}{M} d^{a b c} \delta_{\rho \bar{\sigma}} \delta_{j \bar{i}} J^{c} J^{(\rho \bar{i})} J^{(\bar{\sigma} j)}(w)-2 N i f^{a c e} d^{b c d} \delta_{\rho \bar{\sigma}} t_{j \bar{i}}^{e} J^{d} J^{(\rho \bar{i})} J^{(\bar{\sigma} j)}(w) \\
& +\frac{2\left(4 k^{2}+2 k M+4 k N+M N\right)}{(2 k+M)} d^{b c d} d^{a c e} \delta_{\rho \bar{\sigma}} t_{j \bar{i}}^{e} J^{d} J^{(\rho \bar{i})} J^{(\bar{\sigma} j)}(w) \\
& -4 N(k+N) \sqrt{\frac{M+N}{M N}} d^{a b c} J^{c} \partial J^{u(1)}(w)+2 k N i d^{b c d} f^{a c e} J^{d} \partial J^{e}(w) \\
& \frac{2 k N(2 k+M+2 N)}{(2 k+M)} d^{b c d} d^{a c e} J^{d} \partial J^{e}(w)-\frac{M N}{(2 k+M)} d^{b c d} d^{a c e} J^{d} K^{e}(w) \\
& +N i d^{b c d} f^{a c e} J^{d} K^{e}(w) .
\end{aligned}
$$

Then by substituting appendix (D.2) and appendix (D.3) into appendix (D.1), we obtain the corresponding $a_{5}$ terms explicitly. This is necessary step we should do in order to obtain the final result in (4.45).

\section{D.2 The $\left(a_{13}-a_{12}\right)$ terms of the second order pole}

In particular, in order to calculate the $\left(a_{13}-a_{12}\right)$ terms in appendix (D.1) we should calculate the following first order poles which can be obtained in the OPEs between the first order poles at the point $z$ in the OPE between $K^{a}$ and $J^{(\rho \bar{i})}$ with $\delta_{\rho \bar{\sigma}} t_{j \bar{i}}^{b}$ and the 
current $J^{(\bar{\sigma} j)}(w)$

$$
\begin{aligned}
& \left.\delta_{\rho \bar{\sigma}} t_{j \bar{i}}^{b} k^{k \bar{i}} t_{k \bar{l}}^{a} J^{u(1)} J^{(\rho \bar{l})}(z) J^{(\bar{\sigma} j)}(w)\right|_{\frac{1}{(z-w)}}=k N \delta^{a b} \partial J^{u(1)}(w) \\
& +\frac{1}{2} \sqrt{\frac{M+N}{M N}} i f^{a b c} \delta_{\rho \bar{\sigma}} t_{\bar{j}}^{c} J^{(\rho \bar{i})} J^{(\bar{\sigma} j)}(w)-\frac{1}{2} \sqrt{\frac{M+N}{M N}} d^{a b c} \delta_{\rho \bar{\sigma}} t_{j \bar{i}}^{c} J^{(\rho \bar{i})} J^{(\bar{\sigma} j)}(w) \\
& -\frac{1}{M} \sqrt{\frac{M+N}{M N}} \delta^{a b} \delta_{\rho \bar{\sigma}} \delta_{j \bar{i}} J^{(\rho \bar{i})} J^{(\bar{\sigma} j)}(w)+\sqrt{\frac{M+N}{M N}} N \delta^{a b} J^{u(1)} J^{u(1)}(w) \\
& +\frac{N}{2} i f^{a b c} J^{u(1)} J^{c}(w)-\frac{N}{2} d^{a b c} J^{u(1)} J^{c}(w), \\
& \left.\delta_{\rho \bar{\sigma}} t_{j \bar{i}}^{b} J^{a} J^{(\rho \bar{i})}(z) J^{(\bar{\sigma} j)}(w)\right|_{\frac{1}{(z-w)}}=\frac{1}{2} i f^{a b c} \delta_{\rho \bar{\sigma}} t_{j \bar{i}}^{c} J^{(\rho \bar{i})} J^{(\bar{\sigma} j)}(w) \\
& +\frac{1}{2} d^{a b c} \delta_{\rho \bar{\sigma}} t_{j \bar{i}}^{c} J^{(\rho \bar{i})} J^{(\bar{\sigma} j)}(w)+\frac{1}{M} \delta^{a b} \delta_{\rho \bar{\sigma}} \delta_{j \bar{i}} J^{(\rho \bar{i})} J^{(\bar{\sigma} j)}(w)-N J^{a} J^{b}(w), \\
& \left.\delta_{\rho \bar{\sigma}} t_{j \bar{i}}^{b}\left(i f-\frac{(2 k+M+2 N)}{(2 k+M)} d\right)^{a c d} \delta^{k_{1} \bar{i}} t_{k_{1} \bar{j}_{1}}^{d} J^{c} J^{\left(\rho \bar{j}_{1}\right)}(z) J^{(\bar{\sigma} j)}(w)\right|_{\frac{1}{(z-w)}}= \\
& -\frac{(2 N+M+2 k)}{(M+2 k)} \frac{2}{M} d^{a b c} \delta_{\rho \bar{\sigma}} t_{j \bar{i}}^{c} J^{(\bar{\rho} \bar{i})} J^{(\bar{\sigma} j)}(w)-\delta^{a b} \delta_{\rho \bar{\sigma}} \delta_{j \bar{i}} J^{(\rho \bar{i})} J^{(\bar{\sigma} j)}(w) \\
& +\frac{\left(M^{2}-4\right)(2 k+M+2 N)}{M^{2}(2 k+M)} \delta^{a b} \delta_{\rho \bar{\sigma}} \delta_{j \bar{i}} J^{(\rho \bar{i})} J^{(\bar{\sigma} j)}(w)-\frac{2 N(2 k+M+2 N)}{M(2 k+M)} d^{a b c} \partial J^{c}(w) \\
& -\frac{2 N\left(-4 k+M^{2} N-2 M-4 N\right)}{M(2 k+M)} \sqrt{\frac{M+N}{M N}} \delta^{a b} \partial J^{u(1)}(w) \\
& +\frac{2 N(2 k+M+2 N)}{M(2 k+M)} d^{a b c} \partial J^{c}(w) \\
& -\left(i f-\frac{(2 k+M+2 N)}{(2 k+M)} d\right)^{a c d}\left(\sqrt{\frac{M+N}{M N}} N \delta^{b d} J^{c} J^{u(1)}-\frac{N}{2}(i f+d)^{e b d} J^{c} J^{e}\right. \\
& \left.+\frac{1}{2} \sqrt{\frac{M+N}{M N}} N(i f+d)^{b d c} \partial J^{u(1)}+k N \delta^{b d} \partial J^{c}\right)(w), \\
& \left.\delta_{\rho \bar{\sigma}} t_{j \bar{i}}^{b} \bar{i}^{k_{1} \bar{i}} \delta_{\sigma_{2} \overline{\sigma_{1}}}\left(t^{\alpha}\right)^{\overline{\sigma_{1}} \rho} t_{k_{1} \bar{j}_{1}}^{a} J^{\alpha} J^{\left(\sigma_{2} \bar{j}_{1}\right)}(z) J^{(\bar{\sigma} j)}(w)\right|_{\frac{1}{(z-w)}}=-\frac{\left(N^{2}-1\right)}{M N} \delta^{a b}\left(\delta_{\rho \bar{\sigma}} \delta_{j \bar{i}} J^{(\rho \bar{i})} J^{(\bar{\sigma} j)}\right. \\
& \left.-\sqrt{\frac{M+N}{M N}} N M \partial J^{u(1)}\right)(w)-\frac{\left(N^{2}-1\right)}{2 N}(i f+d)^{b a c}\left(t_{j \bar{i}}^{c} \delta_{j \bar{i}} J^{(\rho \bar{i})} J^{(\bar{\sigma} j)}+N \partial J^{c}\right)(w) \\
& +\delta^{a b} J^{\alpha} J^{\alpha}(w)-\sqrt{\frac{M+N}{M N}}\left(N^{2}-1\right) \delta^{a b} \partial J^{u(1)}(w)+\frac{1}{2}\left(N^{2}-1\right)(-i f+d)^{a b c} \partial J^{c}(w) .
\end{aligned}
$$

Note that the first order pole of the OPE between $\delta_{k \bar{l}}\left(t^{a}\right)^{\bar{i} k} \partial J^{(\rho \bar{l})}(z)$ and $J^{(\bar{\sigma} j)}(w)$ is zero. Then it is obvious to obtain the corresponding $\left(a_{13}-a_{12}\right)$ terms simply by differentiating these appendix (D.4) with respect to the variable $w$. We can easily calculate the second order poles of the OPE between $K^{a}$ and $\partial J^{(\bar{\sigma} j)}$ from (3.1) by differentiating the first relation with respect to the variable $w$ consisting of five terms. 
Finally, we obtain the $\left(a_{13}-a_{12}\right)$ terms as follows:

$$
\begin{aligned}
& \frac{2\left(k^{2}-1\right)(2 k+M+N)}{k(2 k+M)} \delta_{\rho \bar{\sigma}}\left(\frac{1}{M} \delta^{b a} \delta_{j \bar{i}}(w)+\frac{1}{2}(i f+d)^{b a c} t_{j \bar{i}}^{c}\right) J^{(\rho \bar{i})} \partial J^{(\bar{\sigma} j)}(w) \\
& +\frac{2\left(4 k^{3}+2 k^{2} M+3 k^{2} N+k M N-2 k-M-N\right)}{k(2 k+M)} \\
& \times \delta_{\rho \bar{\sigma}}\left(\frac{1}{M} \delta^{a b} \delta_{j \bar{i}}+\frac{1}{2}(i f+d)^{a b c} t_{j \bar{i}}^{c}\right) J^{(\rho \bar{i})} \partial J^{(\bar{\sigma} j)}(w) \\
& -2 N(k+N) \sqrt{\frac{M+N}{M N}} \partial^{2} J^{u(1)}(w)-N i f^{a b c} t_{j \bar{i}}^{c} \delta_{\rho \bar{\sigma}} \partial\left(J^{(\rho \bar{i})} J^{(\bar{\sigma} j)}\right)(w) \\
& +\frac{\left(2 k^{2} N+k M N+4 k+2 M+2 N\right)}{k(2 k+M)} d^{a b c} t_{j \bar{i}}^{c} \delta_{\rho \bar{\sigma}} \partial\left(J^{(\rho \bar{i})} J^{(\bar{\sigma} j)}\right)(w) \\
& +\frac{4\left(k^{2} N+k M N+2 k+M+N\right)}{k M(2 k+M)} \delta^{a b} \delta_{\rho \bar{\sigma}} \delta_{j \bar{i}} \partial\left(J^{(\rho \bar{i})} J^{(\bar{\sigma} j)}\right)(w) \\
& -\frac{2(k+N)(M+N)}{k M} \delta^{a b} \partial\left(J^{u(1)} J^{u(1)}\right)(w)-\frac{N^{2}}{k} \sqrt{\frac{M+N}{M N}} i f^{a b c} \partial\left(J^{u(1)} J^{c}\right)(w) \\
& +\frac{N\left(4 k^{2}+2 k M+4 k N+M N\right)}{k(2 k+M)} \sqrt{\frac{M+N}{M N}} d^{a b c} \partial\left(J^{u(1)} J^{c}\right)(w) \\
& -\frac{2 N(k+M+N)}{k M} \partial\left(J^{a} J^{b}\right)(w)+\frac{k N(2 k+M+2 N)}{(2 k+M)} d^{a b c} \partial^{2} J^{c}(w) \\
& +\frac{N}{2}\left(i f-\frac{(2 k+M+2 N)}{(2 k+M)} d\right)^{a c d}(i f+d)^{e b d} \partial\left(J^{c} J^{e}\right)(w)+k N i f^{a b c} \partial^{2} J^{c}(w) \\
& -2 \delta^{a b} \partial\left(J^{\alpha} J^{\alpha}\right)(w) \\
& +\frac{2}{k}(k+N) \sqrt{\frac{M+N}{M N}}\left[\delta_{\rho \bar{\sigma}}\left(\frac{1}{M} \delta^{a b} \delta_{j \bar{i}}+\frac{1}{2}(i f+d)^{a b c} t_{j \bar{i}}^{c}\right) J^{u(1)} J^{(\rho \bar{i})} J^{(\bar{\sigma} j)}\right. \\
& \left.-\sqrt{\frac{M+N}{M N}} \delta_{\rho \bar{\sigma}}\left(\frac{1}{M} \delta^{a b} \delta_{j \bar{i}}+\frac{1}{2}(i f+d)^{a b c} t_{j \bar{i}}^{c}\right) \partial J^{(\rho \bar{i})} J^{(\bar{\sigma} j)}\right](w) \\
& -\frac{2}{k M}(k+M+N)\left[\delta_{\rho \bar{\sigma}} t_{j \bar{i}}^{b} J^{a} J^{(\rho \bar{i})} J^{(\bar{\sigma} j)}\right. \\
& \left.+\delta_{\rho \bar{\sigma}}\left(\frac{1}{M} \delta^{a b} \delta_{j \bar{i}}+\frac{1}{2}(i f+d)^{b a c} t_{j \bar{i}}^{c}\right) \partial J^{(\rho \bar{i})} J^{(\bar{\sigma} j)}\right](w) \\
& -\left[\left(i f+\frac{(2 k+M+2 N)}{(2 k+M)} d\right)^{a c d} \delta_{\rho \bar{\sigma}}\left(\frac{1}{M} \delta^{d b} \delta_{j \bar{i}}+\frac{1}{2}(i f+d)^{d b e} t_{j \bar{i}}^{e}\right) J^{c} J^{(\rho \bar{i})} J^{(\bar{\sigma} i)}\right. \\
& +\left(i f+\frac{(2 k+M+2 N)}{(2 k+M)} d\right)^{a c d} \delta_{\rho \bar{\sigma}} \\
& \left.\times\left(\frac{1}{M} \delta^{b c} t_{j \bar{i}}^{d}+\frac{1}{2 M} \delta^{d e}(i f+d)^{b c e} \delta_{j \bar{i}}+\frac{1}{4}(i f+d)^{b c e}(i f+d)^{d e f} t_{j \bar{i}}^{f}\right) \partial J^{(\bar{\rho} \bar{i})} J^{(\bar{\sigma} j)}\right](w) \\
& +2\left[\left(t^{\alpha}\right)^{\rho \bar{\sigma}}\left(\frac{1}{M} \delta^{a b} \delta_{j \bar{i}}+\frac{1}{2}(i f+d)^{a b c} t_{j \bar{i}}^{c}\right) J^{\alpha} J^{(\bar{\rho} \bar{i})} J^{(\bar{\sigma} k)}\right. \\
& \left.-\left(N-\frac{1}{N}\right)\left(\frac{1}{M} \delta^{a b} \delta_{j \bar{i}}+\frac{1}{2}(i f+d)^{a b c} t_{j \bar{i}}^{c}\right) \delta_{\rho \bar{\sigma}} \delta_{j \bar{i}} \partial J^{(\rho \bar{i})} J^{(\bar{\sigma} j)}\right](w)+N i f^{a b c} \partial K^{c}(w),
\end{aligned}
$$

where the last four relations in appendix (B.1) are used here in appendix (D.5). 
Therefore, we will obtain the final second order pole by collecting all the relevant terms from appendix (D.2), appendix (D.3) and appendix (D.5) explicitly. It seems that they have rather complicated coset operators. However, the second order pole can be written in simple form as the one in (4.45).

\section{D.3 The relations between the remaining coefficients of $W^{(3)}(w)$ in the second order pole}

In (4.9), we have identified the coefficient of $W^{(3)}(w)$ in the second order pole of the OPE between $K^{a}(z) P^{b}(w)$ and we present the remaining terms of the neutral spin-3 current including the $b_{3}$ term as follows:

$$
\begin{aligned}
& \frac{a_{1}}{b_{1}} b_{3}-\frac{4(k+N)(M+N)}{k M} a_{7}=0 \\
& \frac{a_{1}}{b_{1}} b_{4}-\frac{2(2 k+N)}{k} \sqrt{\frac{M+N}{M N}} a_{1}-4 a_{7}=0 \\
& 2 \frac{a_{1}}{b_{1}} b_{6}+\frac{2(2 k+N)}{M} a_{1}+\frac{2}{M}\left(a_{13}-a_{12}\right)=0 \\
& 2 \frac{a_{1}}{b_{1}} b_{8}+\frac{8(k+N)}{M} a_{7}+\frac{2(k+N)}{k M} \sqrt{\frac{M+N}{M N}}\left(a_{13}-a_{12}\right)=0 \\
& \frac{a_{1}}{b_{1}} b_{9}-M \frac{a_{1}}{b_{1}} b_{6}-(2 k+N) a_{1}-4 a_{13}-2 a_{12}=0 \\
& \frac{a_{1}}{b_{1}} b_{11}-M N \frac{a_{1}}{b_{1}} \sqrt{\frac{M+N}{M N}} b_{8}-4 N(k+N) \sqrt{\frac{M+N}{M N}} a_{7} \\
& \quad-\frac{4(k+N)}{k M}(M+N) a_{13}-\frac{2(k+N)(M+N)}{k M} a_{12}=0 \\
& \frac{a_{1}}{b_{1}} b_{12}+\frac{6(k+N)}{M} a_{12}=0 \\
& \frac{a_{1}}{b_{1}} b_{13}+\frac{6(k+N)}{M} a_{13}=0, \\
& -\frac{M N}{2} \frac{a_{1}}{b_{1}} \sqrt{\frac{M+N}{M N} b_{13}+\frac{a_{1}}{b_{1}} b_{14}-2 N(k+N) \sqrt{\frac{M+N}{M N}} a_{13}} \\
& -(k+N) \sqrt{\frac{M+N}{M N}} a_{12}=0 .
\end{aligned}
$$

It is easy to observe that the above relations appendix (D.6) are satisfied by substituting (2.22), (2.32) and (7.7).

\section{D.4 The relations between the remaining coefficients of $P^{b}(w)$ in the second order pole}

In (4.12), the structure of charged spin-3 current in the second order pole of the OPE $K^{a}(z) P^{b}(w)$ is found and we present the remaining terms in the charged spin-3 current 
including the $a_{2}$ term as follows:

$$
\begin{aligned}
& -\left[\frac{k(3 k+2 M)(2 k+M+2 N)}{(k+M)(2 k+M)}\right] a_{1}+\frac{2 k(2 k+M+N)}{(2 k+M)} a_{1}+4 M a_{5}-a_{12}+a_{13}=0, \\
& \frac{2(2 k+M+N)}{(2 k+M)} a_{1}-\left[\frac{k(3 k+2 M)(2 k+M+2 N)}{(k+M)(2 k+M)}\right] a_{2}-4 a_{5}=0, \\
& \frac{2 N\left(4 k^{2}+2 k M+4 k N+M N\right)}{k(2 k+M)} \sqrt{\frac{M+N}{M N}} a_{7}-\left[\frac{k(3 k+2 M)(2 k+M+2 N)}{(k+M)(2 k+M)}\right] a_{4} \\
& -\frac{4(k+N)(M+N)}{k M} a_{5}+\frac{2 M}{k} \sqrt{\frac{N(M+N)}{M}} a_{9}=0, \\
& \frac{2\left(4 k^{2}+2 k M+4 k N+M N\right)}{2 k+M} a_{7}+\frac{4 M(k+N)}{k} \sqrt{\frac{M+N}{M N}} a_{5} \\
& -2\left[\frac{k(3 k+2 M)(2 k+M+2 N)}{(k+M)(2 k+M)}\right] a_{7}+\frac{(k+N)}{k} \sqrt{\frac{M+N}{M N}}\left(a_{13}-a_{12}\right)+2 M a_{9}=0, \\
& -2 M(k+2 N) a_{5}+\frac{\left(6 k^{2}+3 k M+6 k N+2 M N\right)}{2 k+M} a_{12} \\
& -\left[\frac{k(3 k+2 M)(2 k+M+2 N)}{(k+M)(2 k+M)}\right] a_{12}=0, \\
& 2 M(k+2 N) a_{5}+\frac{\left(6 k^{2}+3 k M+6 k N+2 M N\right)}{2 k+M} a_{13} \\
& -\left[\frac{k(3 k+2 M)(2 k+M+2 N)}{(k+M)(2 k+M)}\right] a_{13}=0, \\
& -2 N \sqrt{\frac{M+N}{M N}}(2 k+M+2 N) a_{5}+\frac{N(k+N)}{k} \sqrt{\frac{M+N}{M N}} a_{12} \\
& +\frac{N\left(4 k^{2}+2 k M+4 k N+M N\right)}{k(2 k+M)} \sqrt{\frac{M+N}{M N}} a_{13} \\
& +\left[\frac{k(3 k+2 M)(2 k+M+2 N)}{(k+M)(2 k+M)}\right]\left(\sqrt{\frac{M+N}{M N}} M N a_{8}-a_{14}\right)=0, \\
& \frac{2 M N(k+N)}{k} \sqrt{\frac{M+N}{M N}} a_{5}+\frac{N\left(4 k^{2}+2 k M+4 k N+M N\right)}{2 k+M} a_{7} \\
& +M N a_{9}+\frac{N(2 k+M+2 N)}{2 k+M} \sqrt{\frac{M+N}{M N}} a_{12} \\
& +\frac{N\left(4 k^{2}+2 k M+4 k N+M N\right)}{k(2 k+M)} \sqrt{\frac{M+N}{M N}} a_{13} \\
& -\left[\frac{k(3 k+2 M)(2 k+M+2 N)}{(k+M)(2 k+M)}\right]\left(N a_{7}-a_{15}\right)=0 .
\end{aligned}
$$

The above relations appendix (D.7) are satisfied by substituting (2.22). 


\section{E The first order pole in the $\mathrm{OPE} K^{a}(z) P^{b}(w)$}

The first order pole of the OPE between the charged spin- 2 current and the charged spin-3 current can be obtained

$$
\begin{aligned}
& \left.K^{a}(z) P^{b}(w)\right|_{\frac{1}{(z-w)}}=t_{\rho \bar{\sigma}}^{\alpha} t_{j \bar{i}}^{b} J^{\alpha}\left[\left(\left(K^{a} J^{(\bar{\rho} \bar{i})}\right)_{\text {pole- } 1} J^{(\bar{\sigma} j)}\right)+J^{(\bar{\rho})}\left(K^{a} J^{(\bar{\sigma} j)}\right)_{\text {pole- } 1}\right](w) a_{1} \\
& +i f^{a b c} J^{\alpha} J^{\alpha} J^{c}(w) a_{2} \\
& +\left(i f^{a c d} K^{d} J^{c} J^{b}+i f^{a c e} J^{c} K^{e} J^{b}+i f^{a b e} J^{c} J^{c} K^{e}\right)(w) a_{3} \\
& +i f^{a b c} K^{c} J^{u(1)} J^{u(1)}(w) a_{4} \\
& +\left[i f^{a d e} d^{b c d} K^{e} K^{c}+d^{b c d} J^{d}\left(K^{a} K^{c}\right)_{\text {pole- } 1+}+\right. \\
& +\frac{N}{(2 k+M)}\left(i f^{a d g} d^{c e f} d^{b c d} K^{g} J^{e} J^{f}+i f^{a g e} d^{b c d} d^{c g f} J^{d} K^{e} J^{f}\right) \\
& +i f^{a g e} d^{b c d} d^{c f g} J^{d} J^{f} K^{e} \\
& \left.-\frac{2 N}{k} \sqrt{\frac{M+N}{M N}}\left(i f^{a d e} d^{b c d} K^{e} J^{c} J^{u(1)}+i f^{a c e} d^{b c d} J^{d} K^{e} J^{u(1)}\right)\right](w) a_{5} \\
& +\left[J^{u(1)}\left(K^{a} K^{b}\right)_{\text {pole- } 1}-\frac{2 N}{k} \sqrt{\frac{M+N}{M N}} i f^{a b c} J^{u(1)} K^{c} J^{u(1)}\right. \\
& \left.+\frac{N}{(2 k+M)}\left(i f^{a d e} d^{b d f} J^{u(1)} K^{e} J^{f}+i f^{a d e} d^{b f d} J^{u(1)} J^{f} K^{e}\right)\right](w) a_{7} \\
& +\left[2 i f^{a b c}(k+M+N) K^{c} T+2(k+M+N) J^{b} \partial K^{a}\right. \\
& -2 i f^{a c d} J^{b} J^{c} K^{d}-2 M J^{b} \partial K^{a}-i f^{a b d} K^{d} J^{c} J^{c} \\
& \left.+i f^{a b c} \frac{M}{(k+N)} K^{c} J^{\alpha} J^{\alpha}+i f^{a b c} \frac{(M+N)}{k} K^{c} J^{u(1)} J^{u(1)}\right](w) a_{8} \\
& +\left(i f^{a c e} d^{b c d} J^{u(1)} K^{e} J^{d}+i f^{a d e} d^{b c d} J^{u(1)} J^{c} K^{e}\right)(w) a_{9} \\
& +\left(-f^{a c d} f^{b c e} \partial K^{d} J^{e}+f^{a c d} f^{b c e} \partial J^{e} K^{d}\right)(w) a_{11} \\
& +\delta_{\rho \bar{\sigma}} t_{j \bar{i}}^{b}\left(\left(\left(K^{a} J^{(\rho \bar{i})}\right)_{\text {pole- } 1} \partial J^{(\bar{\sigma} j}\right)+J^{(\rho \bar{i})}\left(K^{a} \partial J^{(\bar{\sigma} j)}\right)_{\text {pole- } 1}\right)(w)\left(a_{13}-a_{12}\right) \\
& +\left[\frac{1}{2} \partial\left(K^{a} K^{b}\right)_{\text {pole- } 1}-\frac{N}{k} \sqrt{\frac{M+N}{M N}} i f^{a b c} \partial\left(K^{c} J^{u(1)}\right)\right. \\
& \left.+\frac{N}{(2 k+M)}\left(i f^{a d e} d^{b d f} \partial\left(K^{e} J^{f}\right)+i f^{a d e} d^{b f d} \partial\left(J^{f} K^{e}\right)\right)\right](w) a_{12} \\
& +i f^{a b c} \partial^{2} K^{c}(w) a_{16} \\
& +\left(\frac{2}{M} \delta^{b c} \delta^{d e}+\frac{2}{M} \delta^{b e} \delta^{c d}+\frac{2}{M} \delta^{b d} \delta^{c e}+\frac{i}{2} f^{b c f} d^{f d e}+\frac{1}{2} d^{b c f} d^{f d e}\right. \\
& \left.+\frac{i}{2} f^{b e f} d^{f c d}+\frac{1}{2} d^{b e f} d^{f c d}+\frac{i}{2} f^{b d f} d^{f c e}+\frac{1}{2} d^{b d f} d^{f c e}\right) \\
& \times\left(i f^{a c f} K^{f} J^{d} J^{e}+i f^{a d f} J^{c} K^{f} J^{e}+i f^{a e f} J^{c} J^{d} K^{f}\right)(w) a_{17} .
\end{aligned}
$$


In this case, from appendix (E.1), we do not have to consider the additional contractions between the operators because we are focusing on the first order pole. We can also further simplify the above expressions by changing the ordering of operators appropriately. Then the above expression appendix (E.1) plays an important role of a new quasi primary spin-4 current $\hat{R}^{c}(w)$ by multiplying $i f^{a b c}$ together with (4.38) and (4.39).

\section{F The second order pole in the $\operatorname{OPE} K^{a}(z) W^{(3)}(w)$}

The second order pole of the OPE between the charged spin- 2 current and the neutral spin-3 current can be described as

$$
\begin{aligned}
& \left.K^{a}(z) W^{(3)}(w)\right|_{\frac{1}{(z-w)^{2}}}=3 M d^{a b c} J^{b} K^{c}(w) b_{2}-2 M J^{u(1)} K^{a}(w) b_{5} \\
& +\left(4(2 k+M+N) t_{\rho, \bar{\sigma}}^{\alpha} t_{j \bar{i}}^{a} J^{\alpha} J^{(\bar{\rho} \bar{i})} J^{(\bar{\sigma} j)}+\frac{4}{k}(2 k+M+N) J^{a} J^{\alpha} J^{\alpha}\right)(w) b_{6} \\
& +\left(i f^{a b c}\left(K^{c} K^{b}\right)_{\mathrm{pole}-1}+\frac{M N}{(2 k+M)} d^{a b c}\left(K^{b} J^{c}+J^{c} K^{b}\right)-\frac{4 M N}{k} \sqrt{\frac{M+N}{M N}} K^{a} J^{u(1)}\right. \\
& \left.+J^{b}\left(K^{a} K^{b}\right)_{\text {pole-2 }}+\frac{M N}{(2 k+M)} d^{a b c} J^{c} K^{b}\right)(w) b_{7}+2(2 k+2 N+M) J^{u(1)} K^{a}(w) b_{8} \\
& +\left(\frac{2\left(k^{2}-1\right)(2 k+M+N)}{k(2 k+M)} \delta_{\rho \bar{\sigma}} \delta^{k \bar{i}} t_{k \bar{l}}^{a} J^{(\rho \bar{l})} \partial J^{(\bar{\sigma} j)}\right. \\
& +\delta_{\rho \bar{\sigma}} \delta_{j \bar{i}}\left(\left(K^{a} J^{(\rho \bar{i})}\right)_{\mathrm{pole}-1} \partial J^{(\bar{\sigma} j)}\right)_{\mathrm{pole}-1} \\
& \left.+\delta_{\rho \bar{\sigma}} \delta_{j \bar{i}} J^{(\rho \bar{i})}\left(K^{a} \partial J^{(\bar{\sigma} j)}\right)_{p o l e-2}\right)(w)\left(b_{13}-b_{12}\right) \\
& +\left(3(k+M+N) \partial K^{a}-\frac{1}{2}\left(2 M \partial K^{a}+i f^{a b c}\left(K^{c} J^{b}+J^{b} K^{c}\right)\right)\right)(w) b_{12} .
\end{aligned}
$$

Moreover, in order to calculate the $\left(b_{13}-b_{12}\right)$ terms the following relations can be used by considering the similar relations as in appendix (D.4)

$$
\begin{aligned}
& \left.\delta_{\rho \bar{\sigma}} \delta_{\bar{j} \bar{i}} \delta^{k \bar{i}} t_{k \bar{l}}^{a} J^{u(1)} J^{(\rho \bar{l})}(z) J^{(\bar{\sigma} j)}(w)\right|_{\frac{1}{(z-w)}}=-\sqrt{\frac{M+N}{M N}} \delta_{\rho \bar{\sigma}} t_{j \bar{i}}^{a} J^{(\rho \bar{i})} J^{(\bar{\sigma} j)}(w) \\
& -N J^{u(1)} J^{a}(w), \\
& \left.\delta_{\rho \bar{\sigma}} \delta_{j \bar{i}} J^{a} J^{(\rho \bar{i})}(z) J^{(\bar{\sigma} j)}(w)\right|_{\frac{1}{(z-w)}}=\delta_{\rho \bar{\sigma}} t_{j \bar{i}}^{a} J^{(\bar{\rho} \bar{i})} J^{(\bar{\sigma} j)}(w) \\
& +k M N \partial J^{a}-\sqrt{\frac{M+N}{M N}} M N J^{a} J^{u(1)}(w),
\end{aligned}
$$




$$
\begin{aligned}
& \left.\delta_{\rho \bar{\sigma} \bar{\sigma}} \delta_{j \bar{i}}\left(i f-\frac{(2 k+M+2 N)}{(2 k+M)} d\right)^{a c d} \delta^{k_{1} \bar{i}} t_{k_{1} \bar{j}_{1}}^{d} J^{c} J^{\left(\rho \overline{j_{1}}\right)}(z) J^{(\bar{\sigma} j)}(w)\right|_{\frac{1}{(z-w)}}= \\
& \quad \frac{2\left(2 k M^{2}-4 k+M^{3}+M^{2} N-2 M-4 N\right)}{M(2 k+M)} \delta_{\rho \bar{\sigma}} t_{j \bar{i}}^{a} J^{(\rho \bar{i})} J^{(\bar{\sigma} j)}(w) \\
& -\left(i f-\frac{(2 k+M+2 N)}{(2 k+M)} d\right)^{a b c} N J^{b} J^{c}(w), \\
& \left.\delta_{\rho \bar{\sigma}} \delta_{j \bar{i}} \delta^{k_{1} \bar{i}} \delta_{\sigma_{2} \bar{\sigma}_{1}}\left(t^{\alpha}\right)^{\overline{\sigma_{1}} \rho} t_{k_{1} \bar{j}_{1}}^{a} J^{\alpha} J^{\left(\sigma_{2} \overline{j_{1}}\right)}(z) J^{(\bar{\sigma} j)}(w)\right|_{\frac{1}{(z-w)}}=-\frac{\left(N^{2}-1\right)}{N} t_{\rho \bar{\sigma}}^{a} \delta_{j \bar{i}} J^{(\rho \bar{i})} J^{(\bar{\sigma} j)}(w) .
\end{aligned}
$$

Then we obtain the $\delta_{\rho \bar{\sigma}} \delta_{j \bar{i}}\left(\left(K^{a} J^{(\rho \bar{i})}\right)_{\text {pole-1 }} \partial J^{(\bar{\sigma} j)}\right)_{\text {pole-1 }}$ in appendix (F.1) by taking the appropriate derivatives in appendix (F.2). By simplifying appendix (F.1), we will end up with the second order pole in (7.10).

\section{G The first order pole in the $\operatorname{OPE} K^{a}(z) W^{(3)}(w)$}

In the $b_{6}$ term of $W^{(3)}$ in (2.25), after moving the second factor to the left in the second term, there exists a $\partial J^{\alpha}$ term. But the OPE with $K^{a}(z)$ does not contribute to nonzero expression. In the $b_{7}$ term of $W^{(3)}$, there exists a $\partial J^{b}$ term by moving the second factor to the left in the second term. We should include this contribution also. In the $b_{8}$ term of $W^{(3)}$, there exists a $\partial J^{u(1)}$ term by moving the second factor to the left in the second term. But the OPE with $K^{a}(z)$ does not contribute to nonzero expression. Finally, in the $b_{13}$ term of $W^{(3)}$, there exists a $\partial^{2} J^{u(1)}$ term, by moving the second factor to the left, which does not contribute to the nonzero result. It is easy to observe that the OPEs between $K^{a}(z)$ and other terms in the $W^{(3)}(w)$ vanish.

Therefore, we summarize the first order pole as follows:

$$
\begin{aligned}
& \left.K^{a}(z) W^{(3)}(w)\right|_{\frac{1}{(z-w)}}=\left(i f^{a b e} K^{e} J^{c} J^{d}+i f^{a c e} J^{b} K^{e} J^{d}+i f^{a d e} J^{b} J^{c} K^{e}\right)(w) d^{b c d} b_{2} \\
& +i f^{a b c} J^{u(1)}\left(K^{c} J^{b}+J^{b} K^{c}\right)(w) b_{5} \\
& +2\left(t_{\rho \bar{\sigma}}^{\alpha} \delta_{j \bar{i}} J^{\alpha}\left[\left(\left(K^{a} J^{(\bar{i})}\right)_{\text {pole-1 }} J^{(\bar{\sigma} j)}\right)+J^{(\rho \bar{i})}\left(K^{a} J^{(\bar{\sigma} j)}\right)_{\text {pole- } 1}\right]\right)(w) b_{6} \\
& +\left(i f^{a b c} N K^{c} \partial J^{b}+i f^{a b c} N J^{b} \partial K^{c}+2 i f^{a b c} t_{j \bar{i}}^{b} \delta_{\rho \bar{\sigma}} K^{c} J^{(\rho \bar{i})} J^{(\bar{\sigma} j)}\right. \\
& \left.+2 t_{j \bar{i}}^{b} \delta_{\rho \bar{\sigma}} J^{b}\left[\left(\left(K^{a} J^{(\bar{\rho} \bar{i})}\right)_{\text {pole-1 }} J^{(\bar{\sigma} j)}\right)+J^{(\bar{\rho} \bar{i})}\left(K^{a} J^{(\bar{\sigma} j)}\right)_{\text {pole-1 } 1}\right]\right)(w) b_{7} \\
& +2 \delta_{\overline{j i}} \delta_{\rho \bar{\sigma}} J^{u(1)}\left(\left(\left(K^{a} J^{(\bar{\rho} \bar{i})}\right)_{\text {pole-1 }} J^{(\bar{\sigma} j)}\right)+J^{(\rho \bar{i})}\left(K^{a} J^{(\bar{\sigma} j)}\right)_{\text {pole- } 1}\right)(w) b_{8} \\
& +\delta_{j \bar{i}} \delta_{\rho \bar{\sigma}}\left(\left(\left(K^{a} \partial J^{(\rho \bar{i})}\right)_{\text {pole- } 1} J^{(\bar{\sigma} j)}\right)+\partial J^{(\bar{\rho} \bar{i})}\left(K^{a} J^{(\bar{\sigma} j)}\right)_{\text {pole- } 1}\right)(w) b_{12} \\
& +\delta_{j \bar{i}} \delta_{\rho \bar{\sigma}}\left(\left(\left(K^{a} J^{(\rho \bar{i})}\right)_{\text {pole- } 1} \partial J^{(\bar{\sigma} j)}\right)+J^{(\bar{\rho} \bar{i})}\left(K^{a} \partial J^{(\bar{\sigma} j)}\right)_{\text {pole- } 1}\right)(w) b_{13},
\end{aligned}
$$


where the four quantities in the above are given, from (3.1), by

$$
\begin{aligned}
& \left.K^{a}(z) J^{(\rho \bar{i})}(w)\right|_{\frac{1}{(z-w)}} \equiv\left(K^{a} J^{(\rho \bar{i})}\right)_{\text {pole- } 1}= \\
& 2(k+N) \delta_{k \bar{j}}\left(t^{a}\right)^{\bar{i} k} \partial J^{(\rho \bar{j})}(w)-\frac{2(k+N)}{k} \sqrt{\frac{M+N}{M N}} \delta^{k \bar{i}} t_{k \bar{j}}^{a} J^{u(1)} J^{(\rho \bar{j})}(w) \\
& +\frac{2}{k M}(k+M+N) J^{a} J^{(\rho \bar{i})}(w)-\left(i f-\frac{(2 k+M+2 N)}{(2 k+M)} d\right)^{a b c} \delta^{k \bar{i}} t_{k \bar{j}}^{c} J^{b} J^{(\rho \bar{j})}(w) \\
& -2 \delta^{k \bar{i}} \delta_{\sigma \overline{\sigma_{1}}}\left(t^{\alpha}\right)^{\overline{\sigma_{1}} \rho} t_{k \bar{j}}^{a} J^{\alpha} J^{(\sigma \bar{j})}(w), \\
& \left(K^{a} J^{(\bar{\sigma} j)}\right)_{\text {pole-1 }}=2(k+N) \delta_{k \overline{k_{1}}}\left(t^{a}\right)^{\overline{k_{1} j}} \partial J^{(\bar{\sigma} k)}(w)+\frac{2(k+N)}{k} \sqrt{\frac{M+N}{M N}} \delta^{j \bar{l}} t_{k \bar{l}}^{a} J^{u(1)} J^{(\bar{\sigma} k)}(w) \\
& -\frac{2}{k M}(k+M+N) J^{a} J^{(\bar{\sigma} j)}(w)-\left(i f+\frac{(2 k+M+2 N)}{(2 k+M)} d\right)^{a b c} \delta^{j \overline{j_{1}}} t_{k \overline{j_{1}}}^{c} J^{b} J^{(\bar{\sigma} k)}(w) \\
& +2 \delta^{j \bar{l}} \delta_{\tau \overline{\sigma_{1}}}\left(t^{\alpha}\right)^{\bar{\sigma} \tau} t_{k \bar{l}}^{a} J^{\alpha} J^{\left(\overline{\sigma_{1}} k\right)}(w) \\
& \left(K^{a} \partial J^{(\rho \bar{i})}\right)_{\text {pole- } 1}=\partial\left[2(k+N) \delta_{k \bar{j}}\left(t^{a}\right)^{\bar{i} k} \partial J^{(\rho \bar{j})}-\frac{2(k+N)}{k} \sqrt{\frac{M+N}{M N}} \delta^{k \bar{i}} t_{k \bar{j}}^{a} J^{u(1)} J^{(\rho \bar{j})}\right. \\
& +\frac{2}{k M}(k+M+N) J^{a} J^{(\rho \bar{i})}-\left(i f-\frac{(2 k+M+2 N)}{(2 k+M)} d\right)^{a b c} \delta^{k \bar{i}} t_{k \bar{j}}^{c} J^{b} J^{(\rho \bar{j})} \\
& \left.-2 \delta^{k \bar{i}} \delta_{\sigma \overline{\sigma_{1}}}\left(t^{\alpha}\right)^{\overline{\sigma_{1}} \rho} t_{k \bar{j}}^{a} J^{\alpha} J^{(\sigma \bar{j})}\right](w), \\
& \left(K^{a} \partial J^{(\bar{\sigma} j)}\right)_{\text {pole- } 1}=\partial\left[2(k+N) \delta_{k \overline{k_{1}}}\left(t^{a}\right)^{\overline{k_{1} j}} \partial J^{(\bar{\sigma} k)}+\frac{2(k+N)}{k} \sqrt{\frac{M+N}{M N}} \delta^{j \bar{l}} t_{k \bar{l}}^{a} J^{u(1)} J^{(\bar{\sigma} k)}\right. \\
& -\frac{2}{k M}(k+M+N) J^{a} J^{(\bar{\sigma} j)}-\left(i f+\frac{(2 k+M+2 N)}{(2 k+M)} d\right)^{a b c} \delta^{j \overline{j_{1}}} t_{k \overline{j_{1}}}^{c} J^{b} J^{(\bar{\sigma} k)} \\
& \left.+2 \delta^{j \bar{l}} \delta_{\tau \overline{\sigma_{1}}}\left(t^{\alpha}\right)^{\bar{\sigma} \tau} t_{k \bar{l}}^{a} J^{\alpha} J^{\left(\overline{\sigma_{1}} k\right)}\right](w) .
\end{aligned}
$$

Note that we should be careful about the normal ordering [23] in the composite operators containing the first and the third of appendix (G.2) in appendix (G.1). For any operators, $A, B, C$, we have the relation $((A B) C)(w)=([(A B), C])(w)+(C(A B))(w)$ where the bracket stands for the normal ordering between the operators.

\section{H Relevant free field realization}

In this section, the free field realization in [15] is reviewed and we comment on its relevance in the context of previous sections. 


\section{H.1 Free field construction}

The generators of $W_{1+\infty}$ algebra [61] are given by $V^{i}(z)$ of spin- $(i+2)$ and the generator of affine $\mathrm{SU}(M)$ algebra is given by the spin-1 current $4 q W^{-1, a}(z)$ where $a=1,2, \cdots,\left(M^{2}-1\right)$ and $q$ is a parameter and is fixed by $q=\frac{1}{4}$. We follow the notation of [15] except that their $N$ corresponds to our $M$. Moreover the additional generators are given by $W^{i, a}(z)$ of spin$(i+2)$ transforming as the adjoint representation of $\mathrm{SU}(M)$. The realization of the algebra is represented by bilinear free fermions. The complex free fermions satisfy the following OPE

$$
\bar{\psi}^{\alpha}(z) \psi^{\beta}(w)=\frac{1}{(z-w)} \delta^{\alpha \beta}+\cdots,
$$

where $\alpha, \beta=1,2, \cdots, M$. Then the level $k=1$ realization is given by the following forms ${ }^{17}$

$$
\begin{aligned}
V^{j}(z) & =\frac{2^{j-1}(j+1) !}{(2 j+1) ! !} q^{j} \sum_{r=0}^{j+1}(-1)^{r}\left(\begin{array}{c}
j+1 \\
r
\end{array}\right)^{2} \partial^{j-r+1} \bar{\psi}^{\alpha} \partial^{r} \psi^{\alpha}(z), \\
W^{j, a}(z) & =\frac{2^{j-1}(j+1) !}{(2 j+1) ! !} q^{j} \sum_{r=0}^{j+1}(-1)^{r}\left(\begin{array}{c}
j+1 \\
r
\end{array}\right)^{2} \partial^{j-r+1} \bar{\psi}^{\alpha} \partial^{r} t_{\alpha \beta}^{a} \psi^{\beta}(z) .
\end{aligned}
$$

Then we can check that the stress energy tensor $V^{0}(z)$ has the central charge $c=M k$ and it becomes $c=M$ by using the fundamental relation in appendices (H.1) and (H.2). The spin-3 operator $V^{1}(z)$ is a quasi primary operator and has the fourth order pole $V^{-1}(w)$ in the OPE with the above stress energy tensor. Moreover, there is a quasi primary spin-4 $V^{2}(z)$ operator.

We can check that there exist two primary spin-1,2 operators $W^{-1, a}(z)$ and $W^{0, a}(z)$. For the quasi primary spin-3 operator $W^{1, a}(z)$, the OPE between the stress energy tensor $V^{0}(z)$ and $W^{1, a}(w)$ has nonzero fourth order pole $W^{-1, a}(w)$.

We consider two cases as follows:

- The OPEs between the nonsinglet currents and singlet current

When we calculate the OPE between $W^{-1, a}(z)$ and $V^{1}(w)$, we observe that the second order pole contains the spin-2 operator $W^{0, a}(w)$. This can be compared to the previous result in (7.1) which eventually becomes zero. The OPE between $W^{0, a}(z)$ and $V^{1}(w)$ implies that the nonzero singular terms are given by $W^{-1, a}(w)$ in the fourth order pole, $3 W^{1, a}(w)$ in the second order pole and $\partial W^{1, a}(w)$ in the first order pole. We can compare this with the one in (7.10) and realize that there are common linear terms in the (quasi) spin-3 operator. We can further calculate the OPE between the spin-3 operator $W^{1, a}(z)$ and other spin-3 operator $V^{1}(w)$. It turns out that there are $4 W^{0, a}(w), 2 \partial W^{0, a}(w), \frac{3}{5} \partial^{2} W^{0, a}(w)+4 W^{2, a}(w)$, and $\frac{2}{15} \partial^{3} W^{0, a}(w)+$ $2 \partial W^{2, a}(w)$ in the fourth, third, second and first order poles respectively. Again by comparing with (7.11), we observe that the same linear terms occur in both cases.

\footnotetext{
${ }^{17}$ In the calculation of OPEs between the free fields in this section we will consider the $M=4$ case in order to see the structure of the algebra and the general calculation for arbitrary $M$ can be done by hand.
} 
- The OPEs between the nonsinglet currents

The OPE between the spin-1 current and the charged spin- 2 current gives us the nonzero second order pole which is given by $\left(\frac{1}{2} d^{a b c} W^{-1, c}+\frac{1}{4} \delta^{a b} V^{-1}\right)(w)$. Moreover, the first order pole gives $i f^{a b c} W^{0, c}(w)$. Note that there exists a spin-1 singlet current. This can be compared to the relation (2.12). Similarly, the OPE between the charged spin-2 current and itself leads to $\frac{1}{2} \delta^{a b}$ in the fourth order pole, $\frac{1}{2} i f^{a b c} W^{-1, c}(w)$ in the third order pole, $\left(\frac{1}{4} i f^{a b c} \partial W^{-1, c}+\frac{1}{2} \delta^{a b} V^{0}+d^{a b c} W^{0, c}\right)(w)$ in the second order pole and $\left(\frac{1}{12} i f^{a b c} \partial^{2} W^{-1, c}+\frac{1}{4} \delta^{a b} \partial V^{0}+\frac{1}{2} d^{a b c} \partial W^{0, c}+i f^{a b c} W^{1, c}\right)(w)$ in the first order pole respectively. Now we observe that when we compare this with (3.34), both cases share the common linear terms at each singular term. The OPE between the spin-1 current and the charged spin-3 current leads to $\frac{1}{3} f^{a b c} W^{-1, c}(w),\left(-\frac{1}{6} f^{a b c} W^{-1, c}+\right.$ $\left.d^{a b c} W^{0, c}\right)(w)$ and $i f^{a b c} W^{1, c}(w)$ in the third, the second and the first order poles respectively. Note that there are nonzero singular terms in the third order and second order poles when we compare with the one in (2.24).

We can also check the OPE between the charged spin-2 current and the charged spin3 current. It turns out that the fourth order pole is $\left(\frac{1}{4} \delta^{a b} V^{-1}-\frac{i}{2} d^{a b c} W^{-1, c}\right)(w)$, the third order pole is $\frac{4}{3} i f^{a b c} W^{0, c}(w)$, the second order pole is $\left(\frac{3}{4} \delta^{a b} V^{1}-\frac{3 i}{2} d^{a b c} W^{1, c}+\right.$ $\left.\frac{1}{3} i f^{a b c} \partial W^{0, c}\right)(w)$ and the first order pole is $\left(\frac{1}{4} \delta^{a b} \partial V^{1}-\frac{i}{2} d^{a b c} \partial W^{1, c}+\frac{1}{15} i f^{a b c} \partial^{2} W^{0, c}+\right.$ $\left.i f^{a b c} W^{2, c}\right)(w)$. In this case, some of the linear terms of this OPE occur in the (4.45). Note the presence of a spin-1 singlet current. Finally, the OPE between the charged spin-3 current and itself provides the following singular terms. ${ }^{18}$

Therefore, we observe that the presence of a neutral spin-1 current with Kronecker delta symbols appears in the OPEs between the nonsinglet currents where the sum of spins of the left hand side is given by odd integer numbers. Although there are some higher order terms which do not appear in the coset realization, we observe that by simply ignoring the above uncharged spin-1 current, all the linear terms in the free field realization arise in the coset realization. One of the lessons from the free field realization is to expect how the new quasi primary operators arise in the specific singular terms of the given OPEs. From this fact we can rearrange each singular term in the coset realization by expecting that there should be a new quasi primary operator at that singular term. If we do not expect a new quasi primary operator, then we should manage to rewrite each singular term in terms of the multiple product of known currents.

\footnotetext{
${ }^{18}$ The sixth order pole is proportional to the Kronecker delta symbols and is given by $\frac{2}{3} \delta^{a b}$. The fifth order pole contains the spin-1 current and is $\frac{2}{3} i f^{a b c} W^{-1, c}(w)$. The fourth order pole contains the two spin2 currents as well as the descendant terms and is given by $\left(\frac{1}{3} i f^{a b c} \partial W^{-1, c}+\delta^{a b} V^{0}+2 d^{a b c} W^{0, c}\right)(w)$. The third order pole has a spin- 3 current as well as various descendant terms and is given by $\left(\frac{1}{9} i f^{a b c} \partial^{2} W^{-1, c}+\right.$ $\left.\frac{1}{2} \delta^{a b} \partial V^{0}+d^{a b c} \partial W^{0, c}+\frac{8}{3} i f^{a b c} W^{1, c}\right)(w)$. The second order pole contains the two kinds of spin- 4 currents and the descendant terms and is $\left(\frac{1}{36} i f^{a b c} \partial^{3} W^{-1, c}+\frac{3}{20} \delta^{a b} \partial^{2} V^{0}+\frac{3}{10} d^{a b c} \partial^{2} W^{0, c}+\frac{4}{3} i f^{a b c} \partial W^{1, c}+\right.$ $\left.\delta^{a b} V^{2}+2 d^{a b c} W^{2, c}\right)(w)$. Finally, the first order pole contains a spin-5 current besides the various descendant terms and is given by $\left(\frac{1}{180} i f^{a b c} \partial^{4} W^{-1, c}+\frac{1}{30} \delta^{a b} \partial^{3} V^{0}+\frac{1}{15} d^{a b c} \partial^{3} W^{0, c}+\frac{8}{21} i f^{a b c} \partial^{2} W^{1, c}+d^{a b c} \partial W^{2, c}+\right.$ $\left.\frac{1}{2} \delta^{a b} \partial V^{2}+i f^{a b c} W^{3, c}\right)(w)$. Note that the relative coefficients appearing in the descendant terms are fixed automatically.
} 


\section{H.2 After decoupling the neutral spin-1 current}

We can construct the nonsinglet and singlet operators which do not have any singular terms in the OPEs with the above neutral spin-1 current and present them as follows:

$$
\begin{aligned}
\hat{V}^{0}(z) & =V^{0}(z)-\frac{1}{8} V^{-1} V^{-1}(z) \\
\hat{V}^{1}(z)= & V^{1}(z)-\frac{1}{2} V^{-1} V^{0}(z)+\frac{1}{24} V^{-1} V^{-1} V^{-1}(z), \\
\hat{W}^{-1, a}(z)= & W^{-1, a}(z) \\
\hat{W}^{0, a}(z)= & W^{0, a}(z)-\frac{1}{4} V^{-1} W^{-1, a}(z), \\
\hat{W}^{1, a}(z)= & W^{1, a}(z)-\frac{1}{2} V^{-1} W^{0, a}(z)+\frac{1}{16} V^{-1} V^{-1} W^{-1, a}(z) \\
& -\frac{3}{10} \hat{V}^{0} W^{-1, a}(z)+\frac{3}{20} \partial^{2} W^{-1, a}(z) .
\end{aligned}
$$

The central charge is given by $c=M-1=3$. The singlet and nonsinglet operators are primary under the new stress energy tensor. Due to the nonlinear terms in the right hand side, we expect that the algebra between these operators leads to the nonlinear terms in the right hand side of the OPEs. Note that the OPE between the charged spin-1 current and the neutral spin- 1 current is regular. The charged and uncharged spin- 4 currents can be determined similarly.

Moreover, the above operators in appendix (H.3) can be written in terms of $W^{-1, a}(z)$ as follows:

$$
\begin{aligned}
\hat{V}^{0}(z) & =\frac{1}{10} W^{-1, a} W^{-1, a}(z), \\
\hat{V}^{1}(z) & =\frac{1}{90} d^{a b c} W^{-1, a} W^{-1, b} W^{-1, c}(z), \\
\hat{W}^{-1, a}(z) & =W^{-1, a}(z), \\
\hat{W}^{0, a}(z)= & \frac{1}{12} d^{a b c} W^{-1, b} W^{-1, c}(z), \\
\hat{W}^{1, a}(z)= & -\frac{2}{25} W^{-1, b} W^{-1, b} W^{-1, a}(z)-\frac{1}{75} \partial^{2} W^{-1, a}(z) \\
& -\frac{9}{100} i f^{a b c} \partial W^{-1, b} W^{-1, c}(z) .
\end{aligned}
$$

Note that in terms of the complex free fermions, the above operators in appendix (H.4) contain the quartic, the sextic, the quadratic, the quartic, and the sextic terms in the free fermions respectively. The OPE between the spin- 1 current and the charged spin- 2 current gives us the nonzero second order pole which is given by $\frac{1}{2} d^{a b c} \hat{W}^{-1, c}(w)$. Moreover, the first order pole gives $i f^{a b c} \hat{W}^{0, c}(w)$. Note that there is no spin-1 singlet current.

The OPE between the charged spin-2 current $\hat{W}^{0, a}(z)$ and itself can be calculated. It turns out that the fourth order pole is given by $\frac{1}{4} \delta^{a b}$, the third order pole is $\frac{1}{4} i f^{a b c} \hat{W}^{-1, c}(w)$, the second order pole is $\left(\frac{1}{8} i f^{a b c} \partial \hat{W}^{-1, c}+\frac{1}{2} \delta^{a b} \hat{V}^{0}+d^{a b c} \hat{W}^{0, c}-\right.$ $\left.\frac{1}{8}\left(\hat{W}^{-1, a} \hat{W}^{-1, b}+\hat{W}^{-1, b} \hat{W}^{-1, a}\right)\right)(w)$. Note that there are nonlinear terms in this pole. Furthermore, the first order pole is given by $\left(\frac{1}{24} i f^{a b c} \partial^{2} \hat{W}^{-1, c}+\frac{1}{4} \delta^{a b} \partial \hat{V}^{0}+\frac{1}{2} d^{a b c} \partial \hat{W}^{0, c}-\right.$ 
$\left.\frac{1}{16} \partial\left(\hat{W}^{-1, a} \hat{W}^{-1, b}+\hat{W}^{-1, b} \hat{W}^{-1, a}\right)\right)(w)$. This implies that there is no new (quasi) primary operator in this OPE. Other OPEs can be determined without any difficulty.

Therefore, although we have decoupled the neutral spin-1 current in the above analysis, the operators in appendix (H.4) do not produce any new (quasi) primary operators due to the property of the fermions. The charged spin- 1 current which generates the affine $\mathrm{SU}(M)$ algebra produces the $W$ algebra between the nonsinglet currents.

Open Access. This article is distributed under the terms of the Creative Commons Attribution License (CC-BY 4.0), which permits any use, distribution and reproduction in any medium, provided the original author(s) and source are credited.

\section{References}

[1] T. Creutzig, Y. Hikida and P.B. Ronne, Extended higher spin holography and Grassmannian models, JHEP 11 (2013) 038 [arXiv: 1306. 0466] [INSPIRE].

[2] T. Creutzig and Y. Hikida, Rectangular W-algebras, extended higher spin gravity and dual coset CFTs, JHEP 02 (2019) 147 [arXiv:1812.07149] [INSPIRE].

[3] S.F. Prokushkin and M.A. Vasiliev, Higher spin gauge interactions for massive matter fields in 3-D AdS space-time, Nucl. Phys. B 545 (1999) 385 [hep-th/9806236] [InSPIRE].

[4] S. Prokushkin and M.A. Vasiliev, 3-D higher spin gauge theories with matter, in 2nd International Seminar on Supersymmetries and Quantum Symmetries: Dedicated to the Memory of Victor I. Ogievetsky, (1998) [hep-th/9812242] [INSPIRE].

[5] M.R. Gaberdiel and R. Gopakumar, An AdS 3 Dual for Minimal Model CFTs, Phys. Rev. D 83 (2011) 066007 [arXiv: 1011.2986] [InSPIRE].

[6] M.R. Gaberdiel and R. Gopakumar, Triality in Minimal Model Holography, JHEP 07 (2012) 127 [arXiv: 1205.2472] [INSPIRE].

[7] M.R. Gaberdiel and R. Gopakumar, Minimal Model Holography, J. Phys. A 46 (2013) 214002 [arXiv: 1207.6697] [INSPIRE].

[8] M. Ammon, M. Gutperle, P. Kraus and E. Perlmutter, Black holes in three dimensional higher spin gravity: A review, J. Phys. A 46 (2013) 214001 [arXiv:1208.5182] [InSPIRE].

[9] R. Blumenhagen, M. Flohr, A. Kliem, W. Nahm, A. Recknagel and R. Varnhagen, $W$ algebras with two and three generators, Nucl. Phys. B 361 (1991) 255 [InSPIRE].

[10] W. Nahm, Algebras of two-dimensional chiral fields and their classification, in Islamabad 1989, Proceedings, Mathematical physics, pp. 283-300 (1989) [INSPIRE].

[11] W. Nahm, Chiral algebras of two-dimensional chiral field theories and their normal ordered products, in Trieste 1989, Proceedings, Recent developments in conformal field theories, pp. 81-84 (1989) [INSPIRE].

[12] C. Ahn and M.H. Kim, The operator product expansion between the 16 lowest higher spin currents in the $\mathcal{N}=4$ superspace, Eur. Phys. J. C 76 (2016) 389 [arXiv:1509.01908] [INSPIRE].

[13] C. Ahn, D.-g. Kim and M.H. Kim, The next 16 higher spin currents and three-point functions in the large $\mathcal{N}=4$ holography, Eur. Phys. J. C 77 (2017) 523 [arXiv:1703.01744] [InSPIRE]. 
[14] C. Ahn, M.R. Gaberdiel and M.H. Kim, The small $\mathcal{N}=4$ superconformal $\mathcal{W}_{\infty}$ algebra, $J$. Phys. A 53 (2020) 395401 [arXiv: 2004.07439] [InSPIRE].

[15] S. Odake and T. Sano, $W_{1+\infty}$ and super- $W_{\infty}$ algebras with $\mathrm{SU}(N)$ symmetry, Phys. Lett. $B$ 258 (1991) 369 [INSPIRE].

[16] K. Thielemans, A Mathematica package for computing operator product expansions, Int. J. Mod. Phys. C 2 (1991) 787 [InSPIRE].

[17] Wolfram Research, Inc., Mathematica, Version 12.1, Champaign, IL (2020).

[18] L. Eberhardt and T. Procházka, The Grassmannian VOA, JHEP 09 (2020) 150 [arXiv:2006.02422] [INSPIRE].

[19] L. Eberhardt and T. Procházka, The matrix-extended $W_{1+\infty}$ algebra, JHEP 12 (2019) 175 [arXiv: 1910.00041] [INSPIRE].

[20] T. Arakawa and A. Molev, Explicit generators in rectangular affine $\mathcal{W}$-algebras of type $A$, Lett. Math. Phys. 107 (2017) 47 [arXiv:1403.1017] [InSPIRE].

[21] P. Bouwknegt and K. Schoutens, W symmetry in conformal field theory, Phys. Rept. 223 (1993) 183 [hep-th/9210010] [INSPIRE].

[22] M. Beccaria, C. Candu and M.R. Gaberdiel, The large $N=4$ superconformal $W_{\infty}$ algebra, JHEP 06 (2014) 117 [arXiv: 1404.1694] [InSPIRE].

[23] F.A. Bais, P. Bouwknegt, M. Surridge and K. Schoutens, Extensions of the Virasoro Algebra Constructed from Kac-Moody Algebras Using Higher Order Casimir Invariants, Nucl. Phys. B 304 (1988) 348 [INSPIRE].

[24] C. Ahn, The Coset Spin-4 Casimir Operator and Its Three-Point Functions with Scalars, JHEP 02 (2012) 027 [arXiv:1111.0091] [INSPIRE].

[25] J.A. de Azcarraga, A.J. Macfarlane, A.J. Mountain and J.C. Perez Bueno, Invariant tensors for simple groups, Nucl. Phys. B 510 (1998) 657 [physics/9706006] [InSPIRE].

[26] K. Schoutens, Yangian symmetry in conformal field theory, Phys. Lett. B 331 (1994) 335 [hep-th/9401154] [INSPIRE].

[27] P. Goddard, A. Kent and D.I. Olive, Unitary Representations of the Virasoro and Supervirasoro Algebras, Commun. Math. Phys. 103 (1986) 105 [INSPIRE].

[28] P. Goddard, A. Kent and D.I. Olive, Virasoro Algebras and Coset Space Models, Phys. Lett. B 152 (1985) 88 [INSPIRE].

[29] F.A. Bais, P. Bouwknegt, M. Surridge and K. Schoutens, Coset Construction for Extended Virasoro Algebras, Nucl. Phys. B 304 (1988) 371 [inSPIRE].

[30] E. Joung, J. Kim, J. Kim and S.-J. Rey, Asymptotic Symmetries of Colored Gravity in Three Dimensions, JHEP 03 (2018) 104 [arXiv:1712.07744] [INSPIRE].

[31] C. Ahn and C. Peng, Chiral Algebras of Two-Dimensional SYK Models, JHEP 07 (2019) 092 [arXiv: 1812.05106] [INSPIRE].

[32] C. Ahn and H. Kim, Spin-5 Casimir operator its three-point functions with two scalars, JHEP 01 (2014) 012 [Erratum ibid. 01 (2014) 174] [arXiv:1308.1726] [INSPIRE].

[33] C. Ahn and M.H. Kim, The $\mathcal{N}=4$ Higher Spin Algebra for Generic $\mu$ Parameter, JHEP 02 (2021) 123 [arXiv : 2009.04852] [inSPIRE]. 
[34] C. Ahn, D.-g. Kim and M.H. Kim, The $\mathcal{N}=4$ coset model and the higher spin algebra, Int. J. Mod. Phys. A 35 (2020) 2050046 [arXiv: 1910.02183] [InSPIRE].

[35] T. Creutzig and Y. Hikida, Rectangular $W$ algebras and superalgebras and their representations, Phys. Rev. D 100 (2019) 086008 [arXiv:1906.05868] [INSPIRE].

[36] M.R. Gaberdiel and T. Hartman, Symmetries of Holographic Minimal Models, JHEP 05 (2011) 031 [arXiv:1101.2910] [INSPIRE].

[37] C.-M. Chang and X. Yin, Higher Spin Gravity with Matter in AdS $S_{3}$ and Its CFT Dual, JHEP 10 (2012) 024 [arXiv:1106.2580] [INSPIRE].

[38] M. Ammon, P. Kraus and E. Perlmutter, Scalar fields and three-point functions in D=3 higher spin gravity, JHEP 07 (2012) 113 [arXiv:1111.3926] [INSPIRE].

[39] T. Creutzig, Y. Hikida and T. Uetoko, Rectangular $W$-algebras of types $\mathrm{SO}(M)$ and $\operatorname{sp}(2 M)$ and dual coset CFTs, JHEP 10 (2019) 023 [arXiv: 1906.05872] [INSPIRE].

[40] C. Ahn, The Large $N$ 't Hooft Limit of Coset Minimal Models, JHEP 10 (2011) 125 [arXiv:1106.0351] [INSPIRE].

[41] M.R. Gaberdiel and C. Vollenweider, Minimal Model Holography for $\mathrm{SO}(2 N)$, JHEP 08 (2011) 104 [arXiv:1106.2634] [INSPIRE].

[42] C. Ahn, The Large N 't Hooft Limit of Kazama-Suzuki Model, JHEP 08 (2012) 047 [arXiv: 1206.0054] [INSPIRE].

[43] C. Ahn, The Operator Product Expansion of the Lowest Higher Spin Current at Finite N, JHEP 01 (2013) 041 [arXiv: 1208.0058] [inSPIRE].

[44] T. Creutzig, Y. Hikida and P.B. Ronne, Higher spin AdS $S_{3}$ holography with extended supersymmetry, JHEP 10 (2014) 163 [arXiv:1406.1521] [INSPIRE].

[45] C. Ahn, The Higher Spin Currents in the $N=1$ Stringy Coset Minimal Model, JHEP 04 (2013) 033 [arXiv: 1211.2589] [INSPIRE].

[46] C. Ahn, Higher Spin Currents with Arbitrary $N$ in the $\mathcal{N}=\infty$ Stringy Coset Minimal Model, JHEP 07 (2013) 141 [arXiv: 1305.5892] [INSPIRE].

[47] C. Ahn and H. Kim, Higher spin currents in the enhanced $\mathcal{N}=3$ Kazama-Suzuki model, JHEP 12 (2016) 001 [arXiv:1607.00728] [INSPIRE].

[48] C. Candu and M.R. Gaberdiel, Supersymmetric holography on AdS $S_{3}$, JHEP 09 (2013) 071 [arXiv: 1203.1939] [INSPIRE].

[49] M. Henneaux, G. Lucena Gómez, J. Park and S.-J. Rey, Super-W $W_{\infty}$ Asymptotic Symmetry of Higher-Spin $A d S_{3}$ Supergravity, JHEP 06 (2012) 037 [arXiv:1203.5152] [INSPIRE].

[50] K. Hanaki and C. Peng, Symmetries of Holographic Super-Minimal Models, JHEP 08 (2013) 030 [arXiv: 1203.5768] [INSPIRE].

[51] C. Candu and M.R. Gaberdiel, Duality in $N=2$ Minimal Model Holography, JHEP 02 (2013) 070 [arXiv:1207.6646] [INSPIRE].

[52] T. Creutzig, Y. Hikida and P.B. Ronne, Three point functions in higher spin $A d S_{3}$ supergravity, JHEP 01 (2013) 171 [arXiv:1211.2237] [INSPIRE].

[53] M.R. Gaberdiel and R. Gopakumar, Large N=4 Holography, JHEP 09 (2013) 036 [arXiv: 1305.4181] [INSPIRE]. 
[54] M.R. Gaberdiel and M. Kelm, The continuous orbifold of $\mathcal{N}=2$ minimal model holography, JHEP 08 (2014) 084 [arXiv: 1406.2345] [INSPIRE].

[55] S. Datta, L. Eberhardt and M.R. Gaberdiel, Stringy $\mathcal{N}=(2,2)$ holography for $A d S_{3}, J H E P$ 01 (2018) 146 [arXiv:1709.06393] [INSPIRE].

[56] L. Eberhardt, M.R. Gaberdiel and I. Rienacker, Higher spin algebras and large $\mathcal{N}=4$ holography, JHEP 03 (2018) 097 [arXiv: 1801.00806] [INSPIRE].

[57] A. Castro, A. Faraggi and I. Osorio, A note on the $\mathcal{N}=2$ super- $\mathcal{W}_{3}$ holographic dictionary, JHEP 12 (2020) 177 [arXiv:2008.05541] [INSPIRE].

[58] M.R. Gaberdiel and R. Gopakumar, Higher Spins 83 Strings, JHEP 11 (2014) 044 [arXiv: 1406.6103] [INSPIRE].

[59] M.R. Gaberdiel and R. Gopakumar, Stringy Symmetries and the Higher Spin Square, J. Phys. A 48 (2015) 185402 [arXiv:1501.07236] [INSPIRE].

[60] M.R. Gaberdiel and R. Gopakumar, String Theory as a Higher Spin Theory, JHEP 09 (2016) 085 [arXiv : 1512.07237] [INSPIRE].

[61] C.N. Pope, L.J. Romans and X. Shen, A New Higher Spin Algebra and the Lone Star Product, Phys. Lett. B 242 (1990) 401 [InSPIRE]. 